\title{
Global 3-group symmetry and 't Hooft anomalies in axion electrodynamics
}

\author{
Yoshimasa Hidaka, ${ }^{a, b, c}$ Muneto Nitta $^{d}$ and Ryo Yokokura ${ }^{a, d}$ \\ ${ }^{a}$ KEK Theory Center, \\ Tsukuba 305-0801, Japan \\ ${ }^{b}$ Graduate University for Advanced Studies (Sokendai), \\ Tsukuba 305-0801, Japan \\ ${ }^{c}$ RIKEN $i T H E M S$, RIKEN, \\ Wako 351-0198, Japan \\ ${ }^{d}$ Department of Physics 85 Research and Education Center for Natural Sciences, \\ Keio University, Hiyoshi 4-1-1, Yokohama, Kanagawa 223-8521, Japan \\ E-mail: hidaka@post.kek.jp, nitta@phys-h.keio.ac.jp, \\ ryokokur@post.kek.jp
}

ABSTRACT: We investigate a higher-group structure of massless axion electrodynamics in $(3+1)$ dimensions. By using the background gauging method, we show that the higherform symmetries necessarily have a global semistrict 3-group (2-crossed module) structure, and exhibit 't Hooft anomalies of the 3-group. In particular, we find a cubic mixed 't Hooft anomaly between 0 -form and 1-form symmetries, which is specific to the highergroup structure.

KeYwords: Effective Field Theories, Global Symmetries, Topological Field Theories, Topological States of Matter

ARXiv EPrint: 2009.14368 


\section{Contents}

1 Introduction 1

2 Higher-form symmetries in axion electrodynamics 4

2.1 Action 4

2.2 Higher-form symmetries 5

2.2.1 $\mathbb{Z}_{N}$ 0-form symmetry 5

2.2.2 Electric $\mathbb{Z}_{N}$ 1-form symmetry 6

$\begin{array}{lll}\text { 2.2.3 Magnetic U(1) 1-form symmetry } & 7\end{array}$

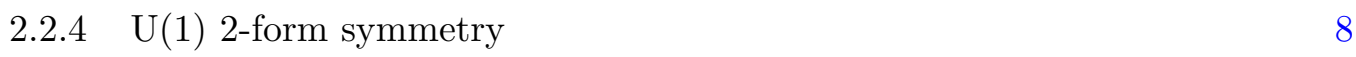

3 Background gauging and 't Hooft anomalies $\quad 8$

3.1 Gauging $\mathbb{Z}_{N}$ 0-form symmetry 8

3.1.1 Constraint on background gauge field 9

$\begin{array}{ll}3.1 .2 & \text { Background gauging as insertion of symmetry generators }\end{array}$

$\begin{array}{ll}3.2 \text { Gauging electric } \mathbb{Z}_{N} \text { 1-form symmetry and U(1) 2-form symmetry } & 10\end{array}$

$\begin{array}{lll}3.2 .1 & \text { Gauging electric } \mathbb{Z}_{N} \text { 1-form symmetry } & 11\end{array}$

$\begin{array}{ll}3.2 .2 \text { Gauging U(1) 2-form symmetry } & 12\end{array}$

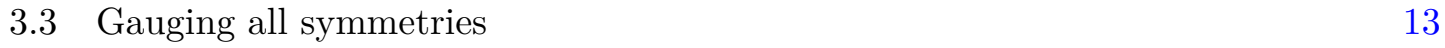

4 Global 3-group symmetry and its gauging in axion electrodynamics $\quad 16$

$\begin{array}{ll}4.1 \text { Correlation functions of symmetry generators } & 16\end{array}$

$\begin{array}{lll}4.2 & \text { Global 3-group symmetry for axion electrodynamics } & 17\end{array}$

$\begin{array}{ll}4.3 \text { Gauging 3-group symmetry } & 19\end{array}$

$\begin{array}{lll}5 & \text { Summary and discussion } & 21\end{array}$

A 't Hooft loop and worldsheet of axionic string 22

$\begin{array}{lll}\text { A.1 't Hooft loop } & 22\end{array}$

A.2 Worldsheet of axionic string 23

B Correlation functions $\quad \mathbf{2 5}$

$\begin{array}{lll}\text { B.1 } \mathbb{Z}_{N} \text { 0-form transformation } & 25\end{array}$

B.2 $\mathbb{Z}_{N}$ 1-form transformation $\quad 26$

B.3 Correlation functions of symmetry generators 26

B.3.1 Correlation function of 0-form and 1-form symmetry generators 27

$\begin{array}{ll}\text { B.3.2 Correlation function of 1-form symmetry generators } & 27\end{array}$

B.3.3 Correlation function of 0 -form and 2-form symmetry generators 28

$\begin{array}{lr}\text { C 3-group gauge theory } & 29\end{array}$

$\begin{array}{lll}\text { C.1 Axiom of 3-group } & 29\end{array}$

C.2 Example of 3-group 30

C.3 Lie algebra of 3-group $\quad 32$

C.4 3-group gauge theory 33 
D Diagrammatic expression of 3-group

D.1 Elements of groups as topological objects 34

D.2 $\partial_{1}$ and $\partial_{2}$ : taking interior of topological objects $\quad 35$

D.3 Action of $G$ : enclosing elements by surfaces 36

D.4 Peiffer lifting: braiding of elements of $H$

D.5 $(L, H)$ as 2-group $\quad 39$

D.6 Global 3-group symmetry and symmetry generators 40

D.6.1 Symmetry transformations 41

D.6.2 Diagrammatic expressions of symmetry transformations 41

D.6.3 Diagrammatic expression of actions 43

D.6.4 Diagrammatic expression of Peiffer lifting 43

D.6.5 Symmetry groups parameterizing symmetry generators 44

\section{Introduction}

Axion electrodynamics has been widely investigated from particle physics and cosmology to condensed matter physics. In particle physics, the axion has been introduced to resolve the strong CP problem, and it is a candidate of cold dark matter [1-7] (see also refs. [811] as a review). In condensed matter physics, the axion electrodynamics can describe magneto-electric responses in topological matter [12-14] (see also ref. [15] as a review).

One of the characteristic features of the axion electrodynamics is a topological coupling between the axion and photon. This coupling originates from the chiral anomaly of massive Dirac fermions coupled with them, which modifies the electric Gauss law and the Maxwell-Ampère law [12-14, 16, 17]. Furthermore, this topological coupling leads to non-trivial effects on extended objects in the axion electrodynamics. There are spatially or temporally extended objects such as magnetic monopoles, axionic domain walls, and axionic strings [18-21]. One of the characteristic effects is the Witten effect [22] for the axion due to the modification of the electric Gauss law [16]. If an axionic domain wall encloses a magnetic monopole, electric charges are induced on the axionic domain wall $[12,17]$. This domain wall enclosing the magnetic monopole is called a monopole bag [23, 24].

Another characteristic effect is the so-called anomalous Hall effect for the axion due to the modification of the Maxwell-Ampère law [12-14, 17, 25-29]. If a domain wall is placed in the electric flux background, electric currents are induced on the domain wall whose direction is perpendicular to the electric flux. This effect also arises in the presence of the axionic strings in the electric flux background [13,21,30]. There are induced electric currents whose directions are perpendicular to both of the electric flux and the gradient of the axion. Since the electric current flows to the axionic strings, this effect is related to the so-called anomaly inflow mechanism of axionic strings [31, 32] (see also refs. [33-36]). By these non-trivial phenomena of extended objects due to the topological coupling, the axion electrodynamics has also been investigated as a simple model of string theory [37-39]. 
What are the underlying structures for the above peculiar effects for the extended objects? One of the candidates is the notion of symmetries, giving us non-perturbative, model-independent, and universal understandings of physical phenomena. In fact, the chiral symmetry and its anomaly in the axion electrodynamics are essential notions to understand these effects. However, its symmetry transformation acts on only local fields rather than extended objects. If we try to understand the effects on extended objects by symmetries, it is plausible to consider symmetries whose transformations act on extended objects.

Recently, the notion of symmetries has been generalized to ones for extended objects, called higher-form symmetries [40-42] (see also related topics [43-51]). For $p$-form symmetries $(p=0,1, \ldots, D)$, charged objects are $p$-dimensional, where $D$ is the spacetime dimensions. Symmetry generators acting on the charged objects are $(D-p-1)$-dimensional topological objects, while the conventional symmetries can be understood as 0 -form symmetries, since they act on local 0-dimensional objects, i.e., local fields. Such higher-form symmetries give us new aspects of modern physics. For example, we can understand photons in the pure Maxwell theory as Nambu-Goldstone bosons [42, 52, 53]. Such an interpretation has been generalized to non-relativistic cases as well $[26,54-56]$. Here, a charged object is a 1-dimensional Wilson loop whose vacuum expectation value is finite in the Coulomb phase. Another application of higher-form symmetries is that Abelian topologically ordered phases [57-60] can be regarded as broken phases of higher-form symmetries [42, 47, 49], in which the charged object is a worldline of an anyon. One can further classify phases of gauge theories based on those symmetries [61-73]. Thus, it becomes possible to understand phenomena of extended objects in terms of higher-form symmetries.

A more elegant description of higher-form symmetries can be given by so-called highergroups [74], which are extensions of conventional groups describing ordinary (0-form) symmetries. Here, higher $n$-groups are given by a set of $n$ groups with maps between them. For example, a 2-group is given by a set of two groups $(G, H)$, a map $H \rightarrow G$, and actions of $G$ on $G$ and $H$. The higher-groups have been recently applied to various aspects of theoretical physics, such as higher gauge theories where charged objects are extended [75-88], effective theories of gapped or gapless topological matter [89-98], deformation of current algebra for tensorial currents [99] and quantum chromodynamics [100]. Therefore, higherform symmetries and higher-group structures may provide us with new understandings of the effects in the axion electrodynamics.

In the previous paper by the present authors [101], it was shown that the axion electrodynamics possesses a 0 -form symmetry, an electric 1-form symmetry, a magnetic 1-form symmetry, and a 2-form symmetry. Here, the 0 -form symmetry is a shift symmetry of the axion. The electric and magnetic 1-form symmetries are related to conservation laws of electric and magnetic fluxes, respectively. The 2-form symmetry is the conservation law of the winding number of axionic strings. Furthermore, we found that the higher-form symmetries can have a semistrict 3-group (or 2-crossed module) structure by analyzing the correlation functions of the symmetry generators. We hereafter abbreviate the semistrict 3 -group to the 3 -group for simplicity. The 3 -group is a set of three groups $(G, H, L)$ with maps between them [102]. One of the particular properties of the 3-group is the presence of 
actions of $G$ on $G, H, L$. Another property is that there must be a map from two elements in $H$ to $L$, which is called the Peiffer lifting.

In this paper, we investigate the higher-group structure in the $(3+1)$-dimensional axion electrodynamics in more detail by introducing background gauge fields corresponding to the higher-form symmetries. The background gauging enables us to describe the 't Hooft anomalies, which are obstructions to dynamical gauging of global symmetries [103-105]. Since the 't Hooft anomalies constrain possible phase structure of a given theory $[61,62,106]$ and describe anomalous phase factors in correlation functions of symmetry generators [42, 107], it is important to determine the 't Hooft anomalies for the axion electrodynamics.

There are at least two methods to establish the background gauging. One is to establish gauged actions with couplings between background gauge fields and symmetry generators $[42,99,100]$. We show that a naive gauging violates the invariance under the transformations of the axion and photon, which should be avoided [99, 107]. The absence of apparent inconsistencies requires modifications of the gauge transformation laws corresponding to the 3-group structure. We should note that this gauging procedure is based on the higher-form symmetries and the gauge invariance, but it does not a priori assume the 3-group structure.

Next, we show that the above background gauging with the modified gauge transformations can be sufficiently described by a 3 -group gauge theory formulated in refs. [79, 82, 83]. To this end, we establish the 3-group gauge theory for the axion electrodynamics. Here, we assume the global 3-group structure in the axion electrodynamics. The gauge transformation laws of the background gauge fields are determined by the basic quantities of the 3 -group rather than the gauge invariance of the axion and photon. By comparing the gauge transformation laws and field strengths, we confirm that these two independent methods result in the same physics.

As a consequence of the background gauging, we determine 't Hooft anomalies of the higher-form symmetries. We find that there are three kinds of the 't Hooft anomalies. One is a mixed 't Hooft anomaly for the axion, which prevents us from a simultaneous gauging of the 0 - and 2-form symmetries. The second is for the photon, which forbids a simultaneous gauging of the two 1-form symmetries. These two anomalies are extensions of previously known anomalies for the axion and photon in the absence of the topological coupling [42]. The third is a cubic 't Hooft anomaly, that is so-called 2-group anomaly [107], which implies the obstruction to the simultaneous gauging of the 0 -form and the 1-form symmetries.

This paper is organized as follows. In section 2, we review the axion electrodynamics and higher-form symmetries in this system in detail. In section 3, we consider the background gauging of the higher-form symmetries that are consistent with the gauge invariance for the dynamical fields. We further determine the 't Hooft anomalies of the higher-form symmetries. In section 4 , we discuss the other gauging procedure, which is based on the 3 -group gauge theory. We show that both of the gauging methods give rise to the same results in the axion electrodynamics. Finally, we summarize this paper in section 5 . We give four appendices. In appendix A, we give explicit forms of the 't Hooft loop and worldsheet of the axionic strings which are charged objects of the magnetic 1-form symmetry and the 2 -form symmetry, respectively. We show detailed derivations of correlation functions used 
in this paper in appendix B. In appendix $\mathrm{C}$, we review the notions of the 3-group, the Lie algebra of the 3 -group, and the 3 -group gauge theories. We also give an intuitive and diagrammatic expression of the 3 -group in appendix D.

\section{Higher-form symmetries in axion electrodynamics}

In this section, we review the higher-form symmetries in $(3+1)$-dimensional axion electrodynamics [101] in detail. In particular, we carefully discuss the symmetry groups for the higher-form symmetries. After giving an action of the massless axion electrodynamics, we show the existence of the higher-form symmetries by the equations of motion (EOM) and Bianchi identities of the axion and photon. We also present the charged objects, symmetry generators, and symmetry groups for the higher-form symmetries.

\subsection{Action}

Here, we give an action of the massless electrodynamics, in which we regard the photon as a gauge field of U(1) gauge symmetry, and the axion as a circle valued pseudo-scalar field. The action has the form [12]

$$
S=-\int_{M_{4}}\left(\frac{v^{2}}{2}|d \phi|^{2}+\frac{1}{2 e^{2}}|d a|^{2}-\frac{N}{8 \pi^{2}} \phi d a \wedge d a\right) .
$$

Here, $\phi$ is the axion, $a$ the photon, $v$ a decay constant of the axion, $e$ a coupling constant of the photon, and $N$ an integer. $|d \phi|^{2}$ and $|d a|^{2}$ denote $d \phi \wedge \star d \phi$ and $d a \wedge \star d a$, where $\star$ is the Hodge star operator. We refer to $M_{4}$ as a $(3+1)$-dimensional spacetime manifold, e.g., the Minkowski spacetime. Throughout this paper, we assume that $M_{4}$ is a spin manifold such that the axion photon coupling term is well-defined. The axion has a $2 \pi$ periodicity at each point $\mathcal{P}$ in the spacetime:

$$
\phi(\mathcal{P})+2 \pi \sim \phi(\mathcal{P}) .
$$

We have assumed that the mass-dimension of the scalar field is normalized as 0 . We regard the periodicity as a redundancy of the axion. In other words, the redundancy can be understood as a (-1)-form gauge symmetry [41, 108, 109]. An invariant operator under the redundant transformation in eq. (2.2) is a point operator,

$$
I\left(q_{\phi E}, \mathcal{P}\right):=e^{i q_{\phi E} \phi(\mathcal{P})},
$$

rather than $\phi(\mathcal{P})$ itself. Here, the invariance requires that $q_{\phi E}$ is an integer. Although $I\left(q_{\phi E}, \mathcal{P}\right)$ is a single-valued function, $\phi(\mathcal{P})$ can be a multi-valued function on a closed loop $\mathcal{C}$ with the winding number,

$$
\int_{\mathcal{C}} d \phi \in 2 \pi \mathbb{Z}
$$

Physically, the nonvanishing winding number implies the existence of a string object with a topologically quantized charge.

The photon is described by a $\mathrm{U}(1)$ 1-form gauge field $a$, which is transformed as

$$
a \rightarrow a+d \lambda .
$$


Here, $\lambda$ is a $\mathrm{U}(1)$ gauge parameter, which satisfies $\lambda(\mathcal{P})+2 \pi \sim \lambda(\mathcal{P})$. Since the gauge parameter is circle valued rather than $\mathbb{R}$ valued, the gauge parameter can have a winding number $\int_{\mathcal{C}} d \lambda \in 2 \pi \mathbb{Z}$. Such a transformation with a nonvanishing winding number is called a large gauge transformation. An operator that is invariant under the large gauge transformation is a Wilson loop,

$$
W\left(q_{a E}, \mathcal{C}\right):=e^{i q_{a E} \int_{\mathcal{C}} a}
$$

where a charge $q_{a E}$ should be an integer. When $\mathcal{C}$ is a boundary of a surface $\mathcal{S}_{\mathcal{C}}$, we can rewrite the Wilson loop by using the Stokes theorem as

$$
W\left(q_{a E}, \mathcal{C}\right)=e^{i q_{a E} \int_{\partial \mathcal{S}_{\mathcal{C}}}{ }^{a}}=e^{i q_{a E} \int_{\mathcal{S}_{\mathcal{C}}} d a}=e^{i q_{a E} \int_{\mathcal{S}_{\mathcal{C}}} f},
$$

where $f=d a$ is the field strength. In general, the field strength is a globally well-defined closed two-form that may not be the exact form, and it is quantized on a closed surface $\mathcal{S}$ as

$$
\int_{\mathcal{S}} f \in 2 \pi \mathbb{Z}
$$

It physically means that there can be a magnetic monopole in the interior of $\mathcal{S}$. This is nothing but the Dirac quantization condition. Throughout this paper, we simply denote the field strength as $d a$ and use the Dirac quantization condition on a closed surface as

$$
\int_{\mathcal{S}} d a \in 2 \pi \mathbb{Z}
$$

bearing in mind that $a$ is not globally well-defined.

\subsection{Higher-form symmetries}

Here, we review higher-form symmetries in this system [101]. In the following, we show that there are four kinds of the higher-form symmetries: $\mathbb{Z}_{N}$ 0-form, electric $\mathbb{Z}_{N}$ 1-form, magnetic U(1) 1-form, and U(1) 2-form symmetries. They are associated with the EOM or Bianchi identities of the axion and photon.

\subsection{1 $\mathbb{Z}_{N}$ 0-form symmetry}

First, we consider the $\mathbb{Z}_{N}$ 0-form symmetry, which is a shift symmetry of the axion. The EOM of the axion, $v^{2} d \star d \phi-\frac{N}{8 \pi^{2}} d a \wedge d a=0$ lead to the following closed current 3 -form and conserved charge,

$$
j_{\phi E}:=-v^{2} \star d \phi-\frac{N}{8 \pi^{2}} a \wedge d a, \quad Q_{\phi E}(\mathcal{V}):=\int_{\mathcal{V}} j_{\phi E} .
$$

Here, $\mathcal{V}$ is a 3 -dimensional closed subspace. The charge $Q_{\phi E}(\mathcal{V})$ is topological: it is invariant under a small deformation $\mathcal{V} \rightarrow \mathcal{V} \cup \partial \Omega_{0}$ with a four-dimensional subspace $\Omega_{0}$, because of the Stokes theorem. A gauge invariant observable given by the current $j_{\phi E}$ is the following unitary object,

$$
U_{\phi E}\left(e^{2 \pi i n_{\phi} / N}, \mathcal{V}\right):=e^{\frac{2 \pi i n_{\phi}}{N}} Q_{\phi E}(\mathcal{V}),
$$


where $e^{2 \pi i n_{\phi} / N} \in \mathbb{Z}_{N}$ parameterizes the topological object. This object is topological meaning that

$$
U_{\phi E}\left(e^{2 \pi i n_{\phi} / N}, \mathcal{V} \cup \partial \Omega_{0}\right)=U_{\phi E}\left(e^{2 \pi i n_{\phi} / N}, \mathcal{V}\right) .
$$

Therefore, $U_{\phi E}$ is a topological unitary object.

One might think that the group parameterizing the symmetry is a continuous group such as $\mathrm{U}(1)$ since there is a conserved current. However, the symmetry group is restricted to $\mathbb{Z}_{N}$ by the large gauge invariance or the Dirac quantization condition of the $\mathrm{U}(1)$ gauge field. This is due to the fact that the current is not gauge invariant, and the conserved charge is not large gauge invariant. Let us consider this problem in detail. We consider a topological unitary object $U_{\phi E}\left(e^{i \alpha_{\phi E}}, \mathcal{V}\right)=e^{i \alpha_{\phi E} Q(\mathcal{V})}$ with a real parameter $\alpha_{\phi E}$. We focus on the gauge variant term $e^{-\frac{i N \alpha_{\phi E}}{8 \pi^{2}} \int_{\mathcal{V}} a \wedge d a}$ in $U_{\phi E}\left(e^{i \alpha_{\phi E}}, \mathcal{V}\right)$ and try to define it by using a gauge invariant integrand $[110,111]$. We define this term by using an auxiliary 4-dimensional subspace $\Omega_{\mathcal{V}}$ with a boundary $\partial \Omega_{\mathcal{V}}=\mathcal{V}$ as

$$
e^{-\frac{i N \alpha_{\phi E}}{8 \pi^{2}} \int_{\mathcal{V}} a \wedge d a}:=e^{-\frac{i N \alpha_{\phi E}}{8 \pi^{2}} \int_{\Omega_{\mathcal{V}}} d a \wedge d a} .
$$

However, the integral has an ambiguity of the choice of $\Omega_{\mathcal{V}}$. We can also define $e^{-\frac{i N \alpha_{\phi E}}{8 \pi^{2}} \int_{\mathcal{V}} a \wedge d a}$ by using another 4-dimensional subspace $\Omega_{\mathcal{V}}^{\prime}$ satisfying $\partial \Omega_{\mathcal{V}}^{\prime}=\mathcal{V}$ as

$$
e^{-\frac{i N \alpha_{\phi E}}{8 \pi^{2}} \int_{\mathcal{V}} a \wedge d a}:=e^{-\frac{i N \alpha_{\phi E}}{8 \pi^{2}} \int_{\Omega_{\mathcal{V}}^{\prime}} d a \wedge d a}
$$

The difference should be invisible, so that we require the following condition,

$$
e^{-\frac{i N \alpha_{\phi E}}{8 \pi^{2}} \int_{\Omega} d a \wedge d a}=1
$$

where $\Omega=\Omega_{\mathcal{V}} \cup\left(-\Omega_{\mathcal{V}}^{\prime}\right)$ is the 4-dimensional closed subspace, and $-\Omega_{\mathcal{V}}^{\prime}$ is the 4-dimensional subspace $\Omega_{\mathcal{V}}^{\prime}$ with an opposite orientation. By the Dirac quantization condition, the integral is $\int_{\Omega} d a \wedge d a \in 2 \cdot(2 \pi)^{2} \mathbb{Z}$ on a spin manifold. Therefore, the parameter $\alpha_{\phi E}$ should satisfy $e^{i \alpha_{\phi E}} \in \mathbb{Z}_{N}{ }^{1}$

The charged object for the symmetry is the 0-dimensional point object in eq. (2.3), and therefore this symmetry is a $\mathbb{Z}_{N} 0$-form symmetry. The symmetry transformation is generated by the topological unitary object and is expressed by the correlation function,

$$
\left\langle U_{\phi E}\left(e^{2 \pi i n_{\phi} / N}, \mathcal{V}\right) I\left(q_{\phi E}, \mathcal{P}\right)\right\rangle=e^{2 \pi i n_{\phi} q_{\phi E} \operatorname{Link}(\mathcal{V}, \mathcal{P}) / N}\left\langle I\left(q_{\phi E}, \mathcal{P}\right)\right\rangle .
$$

Here, the symbol ' \langle\rangle ' denotes a vacuum expectation value (VEV), and $\operatorname{Link}(\mathcal{V}, \mathcal{P}) \in \mathbb{Z}$ is a linking number of $\mathcal{V}$ and $\mathcal{P}$. In appendix B.1, we show the derivation in detail.

\subsubsection{Electric $\mathbb{Z}_{N}$ 1-form symmetry}

Second, we show a $\mathbb{Z}_{N}$ 1-form symmetry originated from the EOM of the photon, $-\frac{1}{e^{2}} d \star$ $d a+\frac{N}{4 \pi^{2}} d \phi \wedge d a=0$, which would imply the conservation of electric fluxes modified by

\footnotetext{
${ }^{1}$ This requirement is the same as the quantization of the Chern-Simons term in $(2+1)$ dimensions [112].
} 
the axion. The closed current 2-form, conserved charge, and topological unitary object are given by

$$
j_{a E}=\frac{1}{e^{2}} \star d a-\frac{N}{4 \pi^{2}} \phi d a, \quad Q_{a E}(\mathcal{S})=\int_{\mathcal{S}} j_{a E}, \quad U_{a E}\left(e^{2 \pi i n_{a} / N}, \mathcal{S}\right)=e^{\frac{2 \pi i n_{a}}{N} Q_{a E}(\mathcal{S})},
$$

respectively. The topological unitary object $U_{a E}$ is parameterized by a $\mathbb{Z}_{N}$ group instead of a $\mathrm{U}(1)$ group due to the gauge variant integrand $\phi d a$. The restriction on the group can be shown as follows. We try to define the integral of the gauge variant term $e^{-\frac{i \alpha_{a E} N}{4 \pi^{2}}} \int_{\mathcal{S}} \phi d a$ in a gauge invariant way, where $\alpha_{a E}$ is a real parameter that will be determined by the large gauge invariance. We define the integral by using a 3-dimensional subspace $\mathcal{V}_{\mathcal{S}}$ as

$$
e^{-\frac{i \alpha_{a E} N}{4 \pi^{2}} \int_{\mathcal{S}} \phi d a}=e^{-\frac{i \alpha_{a E} N}{4 \pi^{2}} \int_{\mathcal{V}_{\mathcal{S}}} d \phi \wedge d a} .
$$

The condition that the integral does not depend on the auxiliary subspace $\mathcal{V}_{\mathcal{S}}$ leads to

$$
e^{-\frac{i \alpha_{a E} N}{4 \pi^{2}} \int_{\mathcal{V}} d \phi \wedge d a}=1
$$

where $\mathcal{V}$ is a 3 -dimensional closed subspace. Since $\int_{\mathcal{V}} d \phi \wedge d a \in(2 \pi)^{2} \mathbb{Z}$, the parameter $\alpha_{a E}$ should be chosen as $e^{i \alpha_{a E}} \in \mathbb{Z}_{N}$.

The charged object for the symmetry is a Wilson loop in eq. (2.6). The transformation law is given by

$$
\left\langle U_{a E}\left(e^{2 \pi i n_{a} / N}, \mathcal{S}\right) W\left(q_{a E}, \mathcal{C}\right)\right\rangle=e^{2 \pi i n_{a} q_{a E} \operatorname{Link}(\mathcal{S}, \mathcal{C}) / N}\left\langle W\left(q_{a E}, \mathcal{C}\right)\right\rangle .
$$

The derivation is shown in appendix B.2. Since the charged object is a 1-dimensional object, the symmetry is a $\mathbb{Z}_{N}$ 1-form symmetry. We refer to this 1-form symmetry as the electric $\mathbb{Z}_{N}$ 1-form symmetry, since the symmetry is related to a conservation of the electric fluxes.

\subsubsection{Magnetic U(1) 1-form symmetry}

Third, we discuss a 1-form symmetry due to the Bianchi identity of the photon, $d d a=0$. The corresponding closed current 2-form, conserved charge, and symmetry generator are given by

$$
j_{a M}=\frac{1}{2 \pi} d a, \quad Q_{a M}(\mathcal{S})=\int_{\mathcal{S}} j_{a M}, \quad U_{a M}\left(e^{i \alpha_{a}}, \mathcal{S}\right)=e^{i \alpha_{a} Q(\mathcal{S})}
$$

respectively. The charged object is an 't Hooft loop $T\left(q_{a M}, \mathcal{C}\right)$, which is a closed worldline of a magnetic monopole. Here, $q_{a M}$ is an integer by the Dirac quantization condition. Note that the explicit form of the 't Hooft loop is shown in appendix A.

If the worldline of the monopole $\mathcal{C}$ is linked with a surface $\mathcal{S}$ of the charge $Q_{a M}(\mathcal{S})$, the charge detects the monopole charge $q_{a M}$ as $Q_{a M}(\mathcal{S})=\frac{1}{2 \pi} \int_{\mathcal{S}} d a=q_{a M} \operatorname{Link}(\mathcal{S}, \mathcal{C})$. In terms of the correlation function of the 't Hooft loop and the symmetry generator, this property can be expressed as a U(1) transformation of the 't Hooft loop by $U_{a M}$ :

$$
\left\langle U_{a M}\left(e^{i \alpha_{a}}, \mathcal{S}\right) T\left(q_{a M}, \mathcal{C}\right)\right\rangle=e^{i \alpha_{a} q_{a M} \operatorname{Link}(\mathcal{S}, \mathcal{C})}\left\langle T\left(q_{a M}, \mathcal{C}\right)\right\rangle .
$$

Since the charged object is a 1-dimensional object, the symmetry is a U(1) 1-form symmetry. Hereafter, we refer to this U(1) 1-form symmetry as the magnetic U(1) 1-form symmetry, since it is related to the conservation law of the magnetic fluxes. 


\begin{tabular}{|c|c|c|c|}
\hline Form & Symmetry generator & Charged object & Group \\
\hline 0 -form $U_{\phi E}$ & $e^{\frac{2 \pi i n_{\phi}}{N} \int_{\mathcal{V}}\left(-v^{2} \star d \phi-\frac{N}{8 \pi^{2}} a \wedge d a\right)}$ & $e^{i q_{\phi E} \phi(\mathcal{P})}$ & $\mathbb{Z}_{N}$ \\
\hline 1-form $U_{a E}$ & $e^{\frac{2 \pi i n_{a}}{N} \int_{\mathcal{S}}\left(\frac{1}{e^{2}} \star d a-\frac{N}{4 \pi^{2}} \phi d a\right)}$ & $e^{i q_{a E} \int_{\mathcal{C}} a}$ & $\mathbb{Z}_{N}$ \\
\hline 1-form $U_{a M}$ & $e^{\frac{i \alpha_{a}}{2 \pi} \int_{\mathcal{S}} d a}$ & $T\left(q_{a M}, \mathcal{C}\right)$ & $\mathrm{U}(1)$ \\
\hline 2-form $U_{\phi M}$ & $e^{\frac{i \alpha_{\phi}}{2 \pi} \int_{\mathcal{C}} d \phi}$ & $V\left(q_{\phi M}, \mathcal{S}\right)$ & $\mathrm{U}(1)$ \\
\hline
\end{tabular}

Table 1. Higher-form symmetries of the massless axion electrodynamics.

\subsubsection{U(1) 2-form symmetry}

Finally, we consider a U(1) 2-form symmetry originated from the Bianchi identity of the axion, $d d \phi=0$. The corresponding current 1-form, conserved charge, and symmetry generator are given by

$$
j_{\phi M}=\frac{1}{2 \pi} d \phi, \quad Q_{\phi M}(\mathcal{C})=\int_{\mathcal{C}} j_{\phi M}, \quad U_{\phi M}\left(e^{i \alpha_{\phi}}, \mathcal{C}\right)=e^{i \alpha_{\phi} Q(\mathcal{C})},
$$

respectively. Here, $e^{i \alpha_{\phi}} \in \mathrm{U}(1)$ parameterizes the symmetry generator. The charged object for the symmetry generator is a worldsheet of the axionic string denoted as $V\left(q_{\phi M}, \mathcal{S}\right)$, where $\mathcal{S}$ is a 2-dimensional closed subspace. In the presence of the axionic string, the winding number of the axion becomes $\int_{\mathcal{C}} d \phi=2 \pi q_{\phi M} \operatorname{Link}(\mathcal{C}, \mathcal{S})$. Note that the explicit form of the worldsheet of the axionic string is shown in appendix A.

We can regard this as a symmetry transformation of the worldsheet of the axionic string, since the axionic string is a source of a topological object $Q_{\phi M}(\mathcal{S})$. In terms of the correlation function, the transformation law is given by

$$
\left\langle U_{\phi M}\left(e^{i \alpha_{\phi}}, \mathcal{C}\right) V\left(q_{\phi M}, \mathcal{S}\right)\right\rangle=e^{i \alpha_{\phi} q_{\phi M} \operatorname{Link}(\mathcal{C}, \mathcal{S})}\left\langle V\left(q_{\phi M}, \mathcal{S}\right)\right\rangle .
$$

We summarize the higher-form symmetries of the massless axion electrodynamics introduced in this section in table 1.

\section{Background gauging and 't Hooft anomalies}

In this section, we consider the background gauging of the higher-form symmetries. We couple the action of the axion electrodynamics with the background gauge fields corresponding to the higher-form symmetries following ref. [42]. We show that the invariance of the gauged action under the gauge transformations of the axion and photon (up to $2 \pi \mathbb{Z}$ ) leads to modifications of the gauge transformation laws of the background gauge fields.

\subsection{Gauging $\mathbb{Z}_{N}$ 0-form symmetry}

First, we couple a background gauge field of the $\mathbb{Z}_{N} 0$-form symmetry, which is introduced as a $\mathrm{U}(1)$ gauge field with a constraint [40]. Although this constraint is already known, we here show the derivation of the constraint explicitly in our case for self-containedness. We also note a relation between the background gauge field and the symmetry generator. 


\subsubsection{Constraint on background gauge field}

First, let us derive the constraint on the background gauge field. The constraint is required by the invariance under the $\mathrm{U}(1)$ gauge transformation of the photon, or equivalently, by the fact that the global symmetry is not $\mathrm{U}(1)$ but $\mathbb{Z}_{N}$. At the linearized level, the background gauging could be done by adding a coupling of the conserved current with a background 1-form gauge field $A_{1}^{\phi E}$,

$$
S_{0, \text { lin. }}=S+\int_{M_{4}} j_{\phi E} \wedge A_{1}^{\phi E}=S-\int_{M_{4}}\left(v^{2} \star d \phi+\frac{N}{8 \pi^{2}} a \wedge d a\right) \wedge A_{1}^{\phi E}
$$

to the action in eq. (2.1). Here, $A_{1}^{\phi E}$ is a $\mathrm{U}(1)$ gauge field that is transformed as

$$
A_{1}^{\phi E} \rightarrow A_{1}^{\phi E}+d \Lambda_{0}^{\phi E}
$$

where $\Lambda_{0}^{\phi E}$ is a $\mathrm{U}(1)$-form gauge parameter that satisfies $\int_{\mathcal{C}} d \Lambda_{0}^{\phi E} \in 2 \pi \mathbb{Z}$ on a closed one-dimensional manifold $\mathcal{C}$. However, the coupling in eq. (3.1) is not invariant under the gauge transformation of the photon $a$ in eq. (2.5). Since the gauge transformation of $a$ leads to the term proportional to $\int_{M_{4}} d \lambda \wedge d a \wedge A_{1}^{\phi E}$, the gauge invariance may be preserved if we impose the flat condition $d A_{1}^{\phi E}=0$, in which $A_{1}^{\phi E}$ is locally expressed as $\alpha d A_{0}^{\phi E}$. Here, $\alpha$ is a parameter that will be determined below.

The gauge transformation of the coupling $\int_{M_{4}} j_{\phi E} \wedge A_{1}^{\phi E}$ becomes a total derivative under the condition, but this total derivative may not vanish under a large gauge transformation. This problem is caused by the presence of the gauge variant integrand $a \wedge d a$ in $S_{0, \text { lin. }}$. In order to discuss the large gauge invariance, we would like to define the term $\frac{N}{8 \pi^{2}} \int_{M_{4}} a \wedge d a \wedge A_{1}^{\phi E}$ by using gauge invariant integrand. We define the term $\frac{N}{8 \pi^{2}} \int_{M_{4}} a \wedge d a \wedge A_{1}^{\phi E}$ on an auxiliary 5-dimensional manifold $X_{5}$ satisfying $\partial X_{5}=M_{4}$ as

$$
\frac{N}{8 \pi^{2}} \int_{M_{4}} a \wedge d a \wedge A_{1}^{\phi E}:=\frac{N}{8 \pi^{2}} \int_{X_{5}} d a \wedge d a \wedge A_{1}^{\phi E}=\frac{N \alpha}{8 \pi^{2}} \int_{X_{5}} d a \wedge d a \wedge d A_{0}^{\phi E} \bmod 2 \pi .
$$

Hereafter, we omit "mod $2 \pi$ " when we discuss the definitions of actions by using 5dimensional manifolds. Note that this definition is a natural extension of the definition of the $(2+1)$-dimensional Chern-Simons term by using $(3+1)$-dimensional integral [110], which we have already discussed in eq. (2.13). While the integrand is manifestly invariant under the gauge transformation of the photon $a$ in eq. (2.5), we have chosen the auxiliary space $X_{5}$. The ambiguity of the choice of the auxiliary space does not exist if the following condition is satisfied:

$$
\frac{N \alpha}{8 \pi^{2}} \int_{Z_{5}} d a \wedge d a \wedge d A_{0}^{\phi E} \in 2 \pi \mathbb{Z}
$$

where $Z_{5}$ is a 5 -dimensional manifold without boundaries. Under the normalization $\int_{\mathcal{C}} d A_{0}^{\phi E} \in 2 \pi \mathbb{Z}$, we have the condition $\alpha=1 / N$. Therefore, the gauge field $A_{1}^{\phi E}$ should satisfy

$$
N A_{1}^{\phi E}=d A_{0}^{\phi E} .
$$

As a consequence, the field strength of $A_{1}^{\phi E}$ vanishes:

$$
F_{2}^{\phi E}:=d A_{1}^{\phi E}=0 .
$$


We refer to the 1-form gauge field with this condition as the $\mathbb{Z}_{N} 1$-form gauge field. This construction is consistent with the fact that the 0 -form global symmetry is a finite group $\mathbb{Z}_{N}$, whose gauge field need to be a flat connection.

We have explained the gauging the $\mathbb{Z}_{N}$ 0-form symmetry at a linearized level of $A_{1}^{\phi E}$, and derived the condition of $A_{1}^{\phi E}$ in eq. (3.5). We can further gauge the $\mathbb{Z}_{N}$ 0-form symmetry at a non-linear level, which can be done as in ordinary gauge theories. We can couple the background gauge field to the action by replacing $d \phi$ with $d \phi-A_{1}^{\phi E}$. Here, the axion is shifted under a gauge transformation of $A_{1}^{\phi E}$ as

$$
A_{1}^{\phi E} \rightarrow A_{1}^{\phi E}+d \Lambda_{0}^{\phi E}, \quad A_{0}^{\phi E} \rightarrow A_{0}^{\phi E}+N \Lambda_{0}^{\phi E}, \quad \phi \rightarrow \phi+\Lambda_{0}^{\phi E} .
$$

We can confirm that the action with the background gauge field is invariant under the gauge transformations of the axion and photon. In order to make the gauge invariance manifest, we define a gauged action by using the 5-dimensional action as

$$
S_{0}=-\int_{M_{4}}\left(\frac{v^{2}}{2}\left|d \phi-A_{1}^{\phi E}\right|^{2}+\frac{1}{2 e^{2}}|d a|^{2}\right)+\frac{N}{8 \pi^{2}} \int_{X_{5}}\left(d \phi-A_{1}^{\phi E}\right) \wedge d a \wedge d a \bmod 2 \pi .
$$

The action, in particular the last term, does not depend on the choice of $X_{5}$, as a consequence of

$$
\frac{N}{8 \pi^{2}} \int_{Z_{5}} d \phi \wedge d a \wedge d a \in 2 \pi N \mathbb{Z}, \quad \text { and } \quad \frac{N}{8 \pi^{2}} \int_{Z_{5}} A_{1}^{\phi E} \wedge d a \wedge d a \in 2 \pi \mathbb{Z} .
$$

Therefore, the gauged action is invariant under the gauge transformations of dynamical fields.

\subsubsection{Background gauging as insertion of symmetry generators}

We can interpret the background gauging as a network of the symmetry generator in the spacetime [42], and the configuration of the symmetry generators is expressed by the background gauge field $A_{1}^{\phi E}$. In particular, we can obtain the symmetry generator $U_{\phi E}\left(e^{2 \pi i n_{\phi} / N}, \mathcal{V}\right)$ by choosing $A_{1}^{\phi E}=\frac{2 \pi n_{\phi}}{N} \delta_{1}(\mathcal{V})$. Here, we have introduced the delta functional $p$-form such that, in $D$-dimensional spacetime $M_{D}$,

$$
\int_{M_{D}} J \wedge \delta_{p}\left(\mathcal{V}_{D-p}\right)=\int_{\mathcal{V}_{D-p}} J
$$

for a $(D-p)$-form $J$ and a $(D-p)$-dimensional manifold $\mathcal{V}_{D-p}$. In the viewpoint of the symmetry generator, the gauge transformation $A_{1}^{\phi E} \rightarrow A_{1}^{\phi E}+d \Lambda_{0}^{\phi E}$ corresponds to a topological deformation $\mathcal{V} \rightarrow \mathcal{V} \cup \partial \Omega_{0}$ in eq. (2.12) by choosing $\Lambda_{0}^{\phi E}=\frac{2 \pi n_{\phi}}{N} \delta_{0}\left(\Omega_{0}\right)$, since $d \delta_{0}\left(\Omega_{0}\right)=\delta_{1}\left(\partial \Omega_{0}\right)$. Note that the condition in eq. (3.5) implies $A_{0}^{\phi E}=2 \pi n_{\phi} \delta_{0}\left(\Omega_{\mathcal{V}}\right)$, since $N A_{1}^{\phi E}=2 \pi n_{\phi} d \delta_{0}\left(\Omega_{\mathcal{V}}\right)$. Here, $\Omega_{\mathcal{V}}$ is a 4 -dimensional subspace whose boundary is $\mathcal{V}$.

\subsection{Gauging electric $\mathbb{Z}_{N}$ 1-form symmetry and $U$ (1) 2-form symmetry}

Next, we gauge the $\mathbb{Z}_{N}$ electric 1-form symmetry. As we see below, we need to gauge the $\mathrm{U}(1)$ 2-form symmetry simultaneously in order to preserve the gauge invariance for the axion. 


\subsubsection{Gauging electric $\mathbb{Z}_{N}$ 1-form symmetry}

Here, we consider the gauging of the electric $\mathbb{Z}_{N} 1$-form symmetry, which can be done by introducing a 2 -form gauge field $B_{2}^{a E}$. Since the global symmetry is parameterized by the $\mathbb{Z}_{N}$ group, there is a similar constraint on $B_{2}^{a E}$. At the linearized level, the coupling would be written as $\int_{M_{4}} j_{a E} \wedge B_{2}^{a E}$. However, this is generally not invariant under the $2 \pi$ shift of $\phi$ due to the term $\int_{M_{4}} \frac{N}{4 \pi^{2}} \phi d a \wedge B_{2}^{a E}$, and the deviation is $\frac{N}{2 \pi} \int_{M_{4}} d a \wedge B_{2}^{a E}$. In order to derive the condition for $B_{2}^{a E}$ such that the coupling $\int_{M_{4}} j_{a E} \wedge B_{2}^{a E}$ is gauge invariant, we define the term $\frac{N}{4 \pi^{2}} \int_{M_{4}} \phi d a \wedge B_{2}^{a E}$ by using a 5-dimensional space as

$$
\frac{N}{4 \pi^{2}} \int_{M_{4}} \phi d a \wedge B_{2}^{a E}=\frac{N}{4 \pi^{2}} \int_{X_{5}} d \phi \wedge d a \wedge B_{2}^{a E} .
$$

The ambiguity of the choice of $X_{5}$ is absent if $N B_{2}^{a E}=d B_{1}^{a E}$ with the normalization $\int_{\mathcal{S}} d B_{1}^{a E} \in 2 \pi \mathbb{Z}$. Therefore, we require that the 2 -form gauge field is constrained by the 1 -form gauge field as

$$
N B_{2}^{a E}=d B_{1}^{a E},
$$

which means that the field strength vanishes,

$$
H_{3}^{a E}:=d B_{2}^{a E}=0 .
$$

At the nonlinear level, the gauging could be done by replacing $d a$ with $d a-B_{2}^{a E}$. The gauged action would be

$$
S_{1 E}=-\int_{M_{4}}\left(\frac{v^{2}}{2}|d \phi|^{2}+\frac{1}{2 e^{2}}\left|d a-B_{2}\right|^{2}\right)+\frac{N}{8 \pi^{2}} \int_{M_{4}} \phi\left(d a-B_{2}^{a E}\right) \wedge\left(d a-B_{2}^{a E}\right) .
$$

The gauge transformation laws of $B_{2}^{a E}, B_{1}^{a E}$, and $a$ are

$$
B_{2}^{a E} \rightarrow B_{2}^{a E}+d \Lambda_{1}^{a E}, \quad B_{1}^{a E} \rightarrow B_{1}^{a E}+N \Lambda_{1}^{a E}, \quad a \rightarrow a+\Lambda_{1}^{a E} .
$$

Here, $\Lambda_{1}^{a E}$ is a 1 -form gauge parameter with the normalization $\int_{\mathcal{S}} d \Lambda_{1}^{a E} \in 2 \pi \mathbb{Z}$. This action can lead to the coupling at the linearized level. However, the non-linear term $\frac{N}{8 \pi^{2}} \int_{M_{4}} \phi B_{2}^{a E} \wedge B_{2}^{a E}$ is not invariant under the $2 \pi$ shift of $\phi$ up to $2 \pi$. In fact, the deviation is $\frac{N}{4 \pi} \int_{M_{4}} B_{2}^{a E} \wedge B_{2}^{a E}=\frac{1}{4 \pi N} \int_{M_{4}} d B_{1}^{a E} \wedge d B_{1}^{a E} \in \frac{2 \pi}{N} \mathbb{Z}$.

We can discuss the problem by using the 5-dimensional action whose integrand is manifestly gauge invariant (see, e.g., recent refs. [113-115]). We can define the gauged topological term in a 5-dimensional spacetime $X_{5}$ as

$$
\frac{N}{8 \pi^{2}} \int_{M_{4}} \phi\left(d a-B_{2}^{a E}\right) \wedge\left(d a-B_{2}^{a E}\right)=\frac{N}{8 \pi^{2}} \int_{X_{5}} d \phi \wedge\left(d a-B_{2}^{a E}\right) \wedge\left(d a-B_{2}^{a E}\right) .
$$

This action is manifestly invariant under the $2 \pi$ shift of $\phi$, but we have chosen an auxiliary 5 -dimensional spacetime $X_{5}$. The gauged action suffers from the ambiguity of the choice of the spacetime:

$$
\frac{N}{8 \pi^{2}} \int_{Z_{5}} d \phi \wedge\left(d a-B_{2}^{a E}\right) \wedge\left(d a-B_{2}^{a E}\right) \in \frac{2 \pi}{N} \mathbb{Z} .
$$

Therefore, we cannot gauge the 1-form symmetry by itself. 


\subsubsection{Gauging U(1) 2-form symmetry}

This problem can be resolved by gauging the U(1) 2-form symmetry simultaneously. This is because the problematic term $\frac{N}{8 \pi^{2}} \int_{X_{5}} d \phi \wedge\left(d a-B_{2}^{a E}\right) \wedge\left(d a-B_{2}^{a E}\right)$ is associated to a closed current 1-form $j_{\phi M}=\frac{1}{2 \pi} d \phi$ of the U(1) 2-form symmetry.

Before discussing the resolution, we consider the gauging of the U(1) 2-form symmetry independently. We introduce a 3 -form gauge field $C_{3}^{\phi M}$, which couples to the closed current of the $\mathrm{U}(1) 2$-form symmetry $j_{\phi M}$ as

$$
S_{2}=S+\int_{M_{4}} j_{\phi M} \wedge C_{3}^{\phi M}=S+\frac{1}{2 \pi} \int_{M_{4}} d \phi \wedge C_{3}^{\phi M}
$$

Here, the 3-form gauge field is normalized by the Dirac quantization condition

$$
\int_{\Omega} d C_{3} \in 2 \pi \mathbb{Z}
$$

where $\Omega$ is a 4 -dimensional closed subspace. The gauge transformation law of the 3 -form gauge field is

$$
C_{3}^{\phi M} \rightarrow C_{3}^{\phi M}+d \Lambda_{2}^{\phi M}
$$

where $\Lambda_{2}^{\phi M}$ is a 2-form gauge parameter that is normalized as $\int_{\mathcal{V}} d \Lambda_{2}^{\phi M} \in 2 \pi \mathbb{Z}$. Since the gauge transformation of the coupling $\int_{M_{4}} j_{\phi M} \wedge C_{3}^{\phi M}$ is a total derivative, the large gauge invariance of the coupling is nontrivial. In order to show the large gauge invariance, we define the coupling on a 5 -dimensional manifold as

$$
S_{2}=S-\frac{1}{2 \pi} \int_{X_{5}} d \phi \wedge d C_{3}^{\phi M}
$$

The gauged action does not depend on the choice of $X_{5}$ :

$$
\frac{1}{2 \pi} \int_{Z_{5}} d \phi \wedge d C_{3}^{\phi M} \in 2 \pi \mathbb{Z}
$$

We now resolve the problem of the gauging of the $\mathbb{Z}_{N} 1$-form symmetry. We can cancel the problematic term $\frac{N}{8 \pi^{2}} \int_{X_{5}} d \phi \wedge B_{2}^{a E} \wedge B_{2}^{a E}$ in eq. (3.14) by modifying the field strength $d C_{3}^{\phi M}$ in eq. (3.21) as

$$
d C_{3}^{\phi M} \rightarrow G_{4}^{\phi M a E}=d C_{3}^{\phi M}+\frac{N}{4 \pi} B_{2}^{a E} \wedge B_{2}^{a E} .
$$

The modification requires an additional gauge transformation law of $C_{3}^{\phi M}$ under $B_{2}^{a E} \rightarrow$ $B_{2}^{a E}+d \Lambda_{1}^{a E}$,

$$
C_{3}^{\phi M} \rightarrow C_{3}^{\phi M}+d \Lambda_{2}^{\phi M}-\frac{N}{2 \pi} \Lambda_{1}^{a E} \wedge B_{2}^{a E}-\frac{N}{4 \pi} \Lambda_{1}^{a E} \wedge d \Lambda_{1}^{a E}
$$

Note that the additional transformation does not violate the normalization of $C_{3}^{\phi M}$,

$$
\int_{\Omega} d\left(d \Lambda_{2}^{\phi M}-\frac{N}{2 \pi} \Lambda_{1}^{a E} \wedge B_{2}^{a E}-\frac{N}{4 \pi} \Lambda_{1}^{a E} \wedge d \Lambda_{1}^{a E}\right) \in 2 \pi \mathbb{Z} .
$$


Eventually, the gauged action can be defined on the 5-dimensions as

$$
\begin{aligned}
S_{1 E, 2}= & -\int_{M_{4}}\left(\frac{v^{2}}{2}|d \phi|^{2}+\frac{1}{2 e^{2}}\left|d a-B_{2}^{a E}\right|^{2}\right)-\frac{1}{2 \pi} \int_{X_{5}} d \phi \wedge G_{4}^{\phi M a E} \\
& +\frac{N}{8 \pi^{2}} \int_{X_{5}} d \phi \wedge\left(d a-B_{2}^{a E}\right) \wedge\left(d a-B_{2}^{a E}\right) .
\end{aligned}
$$

This gauged action has no ambiguity since the problematic term $\frac{N}{8 \pi^{2}} \int_{X_{5}} d \phi \wedge B_{2}^{a E} \wedge B_{2}^{a E}$ is canceled out as

$$
\begin{aligned}
& \frac{N}{8 \pi^{2}} \int_{Z_{5}} d \phi \wedge\left(d a-B_{2}^{a E}\right) \wedge\left(d a-B_{2}^{a E}\right)-\frac{1}{2 \pi} \int_{Z_{5}} d \phi \wedge G_{4}^{\phi M a E} \\
& \quad=\int_{Z_{5}}\left(\frac{N}{8 \pi^{2}} d \phi \wedge d a \wedge d a-\frac{1}{4 \pi^{2}} d \phi \wedge d a \wedge d B_{1}^{a E}-\frac{1}{2 \pi} d \phi \wedge d C_{3}^{\phi M}\right) \in 2 \pi \mathbb{Z} .
\end{aligned}
$$

\subsection{Gauging all symmetries}

We now gauge the $\mathbb{Z}_{N}$ 0-form, electric $\mathbb{Z}_{N}$ 1-form, and U(1) 2-form symmetries. We show that we should simultaneously gauge the magnetic U(1) 1-form symmetry in order to preserve the invariance under the gauge transformation of the photon. In other words, the simultaneous gauging of the $\mathbb{Z}_{N}$ 0-form and $\mathbb{Z}_{N} 1$-form symmetries requires the gauging all of the higher-form symmetries.

Let us try to gauge the $\mathbb{Z}_{N}$ 0-form, electric $\mathbb{Z}_{N}$ 1-form, and U(1) 2-form symmetries. We deform the action $S_{1 E, 2}$ by gauging the $\mathbb{Z}_{N} 0$-form symmetry. The gauged action would be

$$
\begin{aligned}
S_{0,1 E, 2}= & -\int_{M_{4}}\left(\frac{v^{2}}{2}\left|d \phi-A_{1}^{\phi E}\right|^{2}+\frac{1}{2 e^{2}}\left|d a-B_{2}^{a E}\right|^{2}\right)-\frac{1}{2 \pi} \int_{X_{5}}\left(d \phi-A_{1}^{\phi E}\right) \wedge G_{4}^{\phi M a E} \\
& +\frac{N}{8 \pi^{2}} \int_{X_{5}}\left(d \phi-A_{1}^{\phi E}\right) \wedge\left(d a-B_{2}^{a E}\right) \wedge\left(d a-B_{2}^{a E}\right) .
\end{aligned}
$$

However, this gauging depends on the choice of $X_{5}$, or equivalently, the violation of the gauge invariance in the 4-dimensional action. In terms of the 4-dimensional action, the violation of the gauge invariance may be seen as follows. The problematic term is $\frac{N}{4 \pi^{2}} \int_{M_{4}} A_{1}^{\phi E} \wedge a \wedge B_{2}^{a E}$ in eq. (3.28) after the partial integration. The deviation under the large gauge transformation of the photon $a \rightarrow a+d \lambda$ with $\int_{\mathcal{C}} d \lambda \in 2 \pi \mathbb{Z}$ is

$$
\frac{N}{4 \pi^{2}} \int_{M_{4}} A_{1}^{\phi E} \wedge d \lambda \wedge B_{2}^{a E}=\frac{1}{4 \pi^{2} N} \int_{M_{4}} d A_{0}^{\phi E} \wedge d \lambda \wedge d B_{1}^{a E} \in \frac{2 \pi}{N} \mathbb{Z} .
$$

On the other hand, in the 5-dimensional action, the ambiguity of the choice of the 5 -dimensional space can be expressed as

$$
\begin{aligned}
\frac{N}{8 \pi^{2}} \int_{Z_{5}}\left(d \phi-A_{1}^{\phi E}\right) \wedge\left(d a-B_{2}^{a E}\right) \wedge\left(d a-B_{2}^{a E}\right)-\frac{1}{2 \pi} \int_{Z_{5}}\left(d \phi-A_{1}^{\phi E}\right) \wedge G_{4}^{\phi M a E} \bmod 2 \pi \\
=\int_{Z_{5}}\left(\frac{N}{8 \pi^{2}}\left(d \phi-A_{1}^{\phi E}\right) \wedge d a \wedge d a-\frac{1}{4 \pi^{2}}\left(d \phi-A_{1}^{\phi E}\right) \wedge d a \wedge d B_{1}^{a E}\right. \\
\left.\quad-\frac{1}{2 \pi}\left(d \phi-A_{1}^{\phi E}\right) \wedge d C_{3}^{\phi M}\right) \bmod 2 \pi \\
=\int_{Z_{5}}\left(\frac{1}{2 \pi} A_{1}^{\phi E} \wedge d C_{3}^{\phi M}+\frac{1}{4 \pi^{2} N} d a \wedge d A_{0}^{\phi E} \wedge d B_{1}^{a E}\right) \bmod 2 \pi
\end{aligned}
$$


The first term $\int_{Z_{5}} \frac{1}{2 \pi} A_{1}^{\phi E} \wedge d C_{3}^{\phi M} \in \frac{2 \pi}{N} \mathbb{Z}$ in the last line a mixed 't Hooft anomaly of the axion, which just expresses the fact that we cannot regard $A_{1}^{\phi E}$ and $C_{3}^{\phi M}$ as dynamical variables simultaneously [42]. However, the second term,

$$
\int_{Z_{5}} \frac{1}{4 \pi^{2} N} d a \wedge d A_{0}^{\phi E} \wedge d B_{1}^{a E} \in \frac{2 \pi}{N} \mathbb{Z}
$$

is problematic since it depends on the dynamical field (q-number) $a$. We expect that we can eliminate the term by gauging the magnetic $\mathrm{U}(1)$ 1-form symmetry, since the problematic term is proportional to the closed 2-form current of the magnetic U(1) 1-form symmetry $j_{a M}=\frac{1}{2 \pi} d a$.

Before gauging the magnetic U(1) 1-form symmetry in $S_{0,1 E, 2}$, we gauge it in the original action $S$ for simplicity. We introduce a U(1) 2-form gauge field $B_{2}^{a M}$ that is coupled to $j_{a M}$ as $S_{1 M}=S+\frac{1}{2 \pi} \int_{M_{4}} d a \wedge B_{2}^{a M}$. Here, the gauge transformation law of $B_{2}^{a M}$ is

$$
B_{2}^{a M} \rightarrow B_{2}^{a M}+d \Lambda_{1}^{a M},
$$

where $\Lambda_{1}^{a M}$ is a $\mathrm{U}(1) 1$-form gauge parameter normalized as $\int_{\mathcal{S}} d \Lambda_{1}^{a M} \in 2 \pi \mathbb{Z}$. The normalization of $B_{2}^{a M}$ is $\int_{\mathcal{V}} d B_{2}^{a M} \in 2 \pi \mathbb{Z}$ by the Dirac quantization condition. In order to make $S_{1 M}$ manifestly invariant under the large gauge transformations, we again define the coupling in the 5 -dimensional space as

$$
S_{1 M}=S+\frac{1}{2 \pi} \int_{X_{5}} d a \wedge d B_{2}^{a M} .
$$

Now, we gauge the magnetic U(1) 1-form symmetry in $S_{0,1 E, 2}$ to eliminate the problematic term in eq. (3.30). Since we have already gauged the electric U(1) 1-form symmetry in $S_{0,1 E, 2}$, the photon $a$ is shifted under the gauge transformation of $B_{2}^{a E}$. Thus, the field strength $d a$ in eq. (3.33) should be replaced with $d a-B_{2}^{a E}$. Including the term canceling the problematic term in eq. (3.31), we gauge the magnetic 1 -form symmetry by introducing the following term,

$$
\begin{aligned}
S_{0,1 E, 1 M, 2}= & S_{0,1 E, 2}+\frac{1}{2 \pi} \int_{X_{5}}\left(d a-B_{2}^{a E}\right) \wedge\left(d B_{2}^{a M}-\frac{N}{2 \pi} A_{1}^{\phi E} \wedge B_{2}^{a E}\right) \\
= & -\int_{M_{4}}\left(\frac{v^{2}}{2}\left|d \phi-A_{1}^{\phi E}\right|^{2}+\frac{1}{2 e^{2}}\left|d a-B_{2}^{a E}\right|^{2}\right)-\frac{1}{2 \pi} \int_{X_{5}}\left(d \phi-A_{1}^{\phi E}\right) \wedge d C_{3}^{\phi M} \\
& +\frac{N}{8 \pi^{2}} \int_{X_{5}}\left(d \phi-A_{1}^{\phi E}\right) \wedge d a \wedge d a-\frac{N}{4 \pi^{2}} \int_{X_{5}} d \phi \wedge d a \wedge B_{2}^{a E} \\
& +\frac{1}{2 \pi} \int_{X_{5}} d a \wedge B_{2}^{a M}-\frac{1}{2 \pi} \int_{X_{5}} B_{2}^{a E} \wedge\left(d B_{2}^{a M}-\frac{N}{2 \pi} A_{1}^{\phi E} \wedge B_{2}^{a E}\right) .
\end{aligned}
$$

In order to make the gauged action gauge invariant, the gauge transformation law $B_{2}^{a M}$ should be modified as

$$
B_{2}^{a M} \rightarrow B_{2}^{a M}+d \Lambda_{1}^{a M}+\frac{N}{2 \pi} \Lambda_{0}^{\phi E} B_{2}^{a E}-\frac{N}{2 \pi}\left(A_{1}^{\phi E}+d \Lambda_{0}^{\phi E}\right) \wedge \Lambda_{1}^{a E}
$$

with $A_{1}^{\phi E} \rightarrow A_{1}^{\phi E}+d \Lambda_{0}^{\phi E}$ and $B_{2}^{a E} \rightarrow B_{2}^{a E}+d \Lambda_{1}^{a E}$. Note that the modified gauge transformations of $B_{2}^{a M}$ preserve the Dirac quantization condition of $B_{2}^{a M}$ :

$$
\int_{\mathcal{V}} d\left(d \Lambda_{1}^{a M}+\frac{N}{2 \pi} \Lambda_{0}^{\phi E} B_{2}^{a E}-\frac{N}{2 \pi}\left(A_{1}^{\phi E}+d \Lambda_{0}^{\phi E}\right) \wedge \Lambda_{1}^{a E}\right) \in 2 \pi \mathbb{Z} .
$$


Accordingly, the gauge invariant field strength for $B_{2}^{a M}$ is identified as

$$
H_{3}^{a M, \phi E}:=d B_{2}^{a M}-\frac{N}{2 \pi} A_{1}^{\phi E} \wedge B_{2}^{a E} .
$$

By adding the term, the problematic operator-valued ambiguity in eq. (3.30) is now absent,

$$
\begin{aligned}
& \frac{N}{8 \pi^{2}} \int_{Z_{5}}\left(d \phi-A_{1}^{\phi E}\right) \wedge\left(d a-B_{2}^{a E}\right) \wedge\left(d a-B_{2}^{a E}\right)-\frac{1}{2 \pi} \int_{X_{5}}\left(d \phi-A_{1}^{\phi E}\right) \wedge G_{4}^{\phi M a E} \\
& \quad+\frac{1}{2 \pi} \int_{Z_{5}}\left(d a-B_{2}^{a E}\right) \wedge H_{3}^{a M, \phi E} \bmod 2 \pi \\
& =\frac{1}{2 \pi} \int_{Z_{5}} A_{1}^{\phi E} \wedge d C_{3}^{\phi M}-\frac{1}{2 \pi} \int_{Z_{5}} B_{2}^{a E} \wedge d B_{2}^{a M} \\
& \quad+\frac{N}{(2 \pi)^{2}} \int_{Z_{5}} A_{1}^{\phi E} \wedge B_{2}^{a E} \wedge B_{2}^{a E} \bmod 2 \pi
\end{aligned}
$$

The remaining ambiguity in the right-hand side represents the 't Hooft anomalies.

In summary, we have introduced the background gauge fields $\left(A_{1}^{\phi E}, B_{2}^{a E}, B_{2}^{a M}, C_{3}^{\phi M}\right)$, whose action is given in eq. (3.34). The gauge transformation laws are given by eqs. (3.7), (3.15), (3.35), and (3.24), respectively. The field strengths are determined by eqs. (3.6), (3.13), (3.37) and (3.23), respectively. In the next section, we derive the above gauge transformation laws and field strength from the viewpoint of the 3-group gauge theory.

Thus, we have successfully gauged the higher-form symmetries. Furthermore, we have obtained the 't Hooft anomalies for the higher-form symmetries. The first term in eq. (3.38) is the mixed 't Hooft anomalies of the axion, which has been discussed previously. The second term is that of the photon, which prohibits the simultaneous dynamical gauging of the pair of the electric 1-form and magnetic 1-form symmetries. The third one can be identified as the so-called 2-group anomaly [107]. This anomaly means the obstruction to the simultaneous gauging of the $\mathbb{Z}_{N} 0$-form symmetry and the electric $\mathbb{Z}_{N} 1$-form symmetry.

The existence of the 't Hooft anomalies forbids a trivial gapped vacuum. In our case, this requirement is satisfied by the existence of the massless axion and photon. The existence of the massless axion corresponds to the existence of the mixed anomaly between the 0 -form and 2-form symmetries. Likewise, the existence of the massless photon corresponds to the existence of the mixed anomaly between the electric and magnetic 1-form symmetries. If we deform the system with preserving these higher-form symmetries, any trivial gapped vacuum is still forbidden. For example, if a gapped vacuum is realized while preserving the symmetries, we can have topologically ordered phases, whose ground states can be degenerated on a compact spatial manifold.

Further, the existence of the 2-group anomaly implies the existence of a fractionally charged particle where $A_{1}^{\phi E} \wedge B_{2}^{a E}$ is non-zero. Physically, it means that we have a fractionally charged particle on the domain wall if we add the magnetic field through the domain wall, which was proposed in ref. [17].

In order to see this effect, we consider the following partition function given by the gauged action in eq. (3.34),

$$
Z\left[A_{1}^{\phi E}, B_{2}^{a E}, B_{2}^{a M}, C_{3}^{\phi M}, X_{5}\right]=\mathcal{N} \int \mathcal{D}[\phi, a] e^{i S_{0,1 E, 1 M, 2}\left[\phi, a, A_{1}^{\phi E}, B_{2}^{a E}, B_{2}^{a M}, C_{3}^{\phi M}, X_{5}\right]} .
$$


Here, $\mathcal{N}$ is a normalization factor such that $\langle 1\rangle=1$. By setting $A_{1}^{\phi E}=\frac{2 \pi n_{\phi}}{N} \delta_{1}(\mathcal{V}), B_{2}^{a E}=$ $\frac{2 \pi n_{a}}{N} \delta_{2}(\mathcal{S}), B_{2}^{a M}=0$, and $C_{3}^{\phi M}=0$, we obtain

$$
\begin{aligned}
Z\left[\frac{2 \pi n_{\phi}}{N} \delta_{1}(\mathcal{V}), \frac{2 \pi n_{a}}{N} \delta_{2}(\mathcal{S}), 0,0, X_{5}\right] & =\mathcal{N} \int \mathcal{D}[\phi, a] e^{-\frac{i n_{a} n_{\phi}}{N} \int_{M_{4}} a \wedge \delta_{1}(\mathcal{V}) \wedge \delta_{2}(\mathcal{S})} e^{i S[\phi, a]} \\
& =\left\langle e^{-\frac{i n_{a} n_{\phi}}{N} \int_{M_{4}} a \wedge \delta_{1}(\mathcal{V}) \wedge \delta_{2}(\mathcal{S})}\right\rangle,
\end{aligned}
$$

where we have redefined $\phi-\frac{2 \pi n_{\phi}}{N} \delta_{0}(\Omega \mathcal{V}) \rightarrow \phi$ and $a+\frac{2 \pi n_{a}}{N} \delta_{1}\left(\mathcal{V}_{\mathcal{S}}\right) \rightarrow a$ in the path integral. On the right-hand side, we have the term $e^{-\frac{i n_{a} n_{\phi}}{N} \int_{M_{4}} a \wedge \delta_{1}(\mathcal{V}) \wedge \delta_{2}(\mathcal{S})}$ which is given by the term related to the 2-group anomaly $\frac{1}{2 \pi} \int_{X_{5}}\left(d a-B_{2}^{a E}\right) \wedge\left(d B_{2}^{a M}-\frac{N}{2 \pi} A_{1}^{\phi E} \wedge B_{2}^{a E}\right)$. Since $\delta_{1}(\mathcal{V}) \wedge \delta_{2}(\mathcal{S})$ is a delta function 3-form on the closed line $\mathcal{V} \cap \mathcal{S}$, the right-hand side implies the existence of the Wilson loop on $\mathcal{V} \cap \mathcal{S}$ with the fractional charge $-\frac{n_{a} n_{\phi}}{N}$. Note that this fractionally charged particle does not arise as long as we do not consider the intersection of the background fields i.e., the symmetry generators.

\section{Global 3-group symmetry and its gauging in axion electrodynamics}

In this section, we derive the background gauging by a different approach based on the 3 -group gauge theory. First, we review the global 3-group symmetry by the structure of the correlation functions of the symmetry generators [101]. The correlation functions give us ingredients of the 3 -group. Next, we establish the 3 -group gauge theory, which can be formulated for a given 3-group. We should remark that this 3 -group gauge theory is based on a mathematical procedure, independent of the gauging based on the gauge invariance of dynamical fields in the previous section. We confirm that the gauging of this section coincides with the one in the previous section.

\subsection{Correlation functions of symmetry generators}

We review the correlation functions of the symmetry generators [101], which give us the group structure in the higher-form symmetries. This is a natural generalization of current algebra in ordinary quantum field theories. The details of the derivations are summarized in appendix B.3. Note that we only consider the correlation functions of the symmetry generators which are not intersected to each other. Therefore, we do not have to consider fractionally charged objects due to the intersection of the symmetry generators in section 3.3 .

It has been shown that the correlation functions of the symmetry generators are not independent, but related to each other. First, the correlation functions of the 0 -form and electric 1-form symmetry generators induce a magnetic 1-form symmetry generator:

$$
\begin{aligned}
\left\langle U_{\phi E}\left(e^{2 \pi i n_{\phi} / N}, \mathcal{V}\right)\right. & \left.U_{a E}\left(e^{2 \pi i n_{a} / N}, \mathcal{S}\right)\right\rangle \\
= & \left\langle U_{a E}\left(e^{2 \pi i n_{a} / N}, \mathcal{S}\right) U_{a M}\left(e^{-2 \pi i n_{\phi} n_{a} / N}, \Omega \mathcal{V} \cap \mathcal{S}\right)\right\rangle \\
\left\langle U_{\phi E}\left(e^{2 \pi i n_{\phi} / N}, \mathcal{V}\right)\right. & \left.U_{a E}\left(e^{2 \pi i n_{a} / N}, \mathcal{S}\right)\right\rangle \\
& =\left\langle U_{\phi E}\left(e^{2 \pi i n_{\phi} / N}, \mathcal{V}\right) U_{a M}\left(e^{-2 \pi i n_{\phi} n_{a} / N},\left(-\mathcal{V}_{\mathcal{S}}\right) \cap \mathcal{V}\right)\right\rangle
\end{aligned}
$$


where $\Omega_{\mathcal{V}}$ and $\mathcal{V}_{\mathcal{S}}$ are 4- and 3-dimensional subspaces satisfying $\partial \Omega_{\mathcal{V}}=\mathcal{V}$ and $\partial \mathcal{V}_{\mathcal{S}}=\mathcal{S}$, respectively. Here, we have eliminated the 0 -form and electric 1-form symmetry generators by redefining the integral variables of the path integral in eq. (4.1) and eq. (4.2), respectively. The minus sign in $-\mathcal{V}_{\mathcal{S}}$ in eq. $(4.2)$ is due to the minus sign in $\delta_{2}(\mathcal{S})=-d \delta_{1}\left(\mathcal{V}_{\mathcal{S}}\right)$. This sign matches the choice of the background gauge fields $B_{2}=\frac{2 \pi n_{a}}{N} \delta_{2}(\mathcal{S})$ and $B_{1}=-2 \pi n_{a} \delta_{1}\left(\mathcal{V}_{\mathcal{S}_{1}}\right)$ in eq. (3.34).

A physical meaning of these relations in eqs. (4.1) and (4.2) is that the electric flux can be induced by the axionic domain wall in the presence of the magnetic monopole inside the axionic domain wall. Therefore, the correlation functions can be interpreted as the Witten effect of the axion [101]: if the domain wall encloses a magnetic monopole, the domain wall induces the electric flux [12, 17, 23, 24].

Second, we consider the correlation function of the 1-form symmetry generators, which leads to a 2-form symmetry generator:

$$
\left\langle U_{a E}\left(e^{2 \pi i n_{a} / N}, \mathcal{S}_{1}\right) U_{a E}\left(e^{2 \pi i n_{a}^{\prime} / N}, \mathcal{S}_{2}\right)\right\rangle=\left\langle U_{\phi M}\left(e^{2 \pi i n_{a} n_{a}^{\prime} / N},\left(-\mathcal{V}_{\mathcal{S}_{1}}\right) \cap \mathcal{S}_{2}\right) U_{a E}\left(e^{2 \pi i n_{a}^{\prime} / N}, \mathcal{S}_{2}\right)\right\rangle
$$

Here, we have eliminated $U_{a E}\left(e^{2 \pi i n_{a} / N}, \mathcal{S}_{1}\right)$ by the same procedure. The minus sign in $-\mathcal{V}_{\mathcal{S}_{1}}$ is due to the minus sign in $\delta_{2}\left(\mathcal{S}_{1}\right)=-d \delta_{1}\left(\mathcal{V}_{\mathcal{S}_{1}}\right)$. This sign matches the choice of the background gauge fields $B_{2}=\frac{2 \pi}{N}\left(n_{a} \delta_{2}\left(\mathcal{S}_{1}\right)+n_{a}^{\prime} \delta_{2}\left(\mathcal{S}_{2}\right)\right)$ and $B_{1}=-2 \pi\left(n_{a} \delta_{2}\left(\mathcal{V}_{\mathcal{S}_{1}}\right)+\right.$ $\left.n_{a}^{\prime} \delta_{1}\left(\mathcal{V}_{\mathcal{S}_{2}}\right)\right)$ in eq. (3.34).

Physically, the relation in eq. (4.3) means that the magnetic field can be induced by the electric field in the presence of the axionic string. The correlation function represents the anomalous Hall effect for the axion [101]. In the presence of the axionic string and the electric field, the electric current is induced [13, 21, 30]. By the Maxwell-Ampère law, the electric current induces the magnetic field.

Other correlation functions induce no further symmetry generators. For example, one can evaluate the correlation function of the 0-form and 2-form symmetry generators,

$$
\left\langle U_{\phi E}\left(e^{2 \pi i n_{\phi} / N}, \mathcal{V}\right) U_{\phi M}\left(e^{i \alpha_{\phi}}, \mathcal{C}\right)\right\rangle=\left\langle U_{\phi M}\left(e^{i \alpha_{\phi}}, \mathcal{C}\right)\right\rangle .
$$

We would like to discuss a mathematical structure behind these correlation functions. One candidate is a 2-group, which is roughly given by two groups and maps between them. In terms of a 2-group, we may describe the correlation function of 0- and 1-form symmetry generators. However, the correlation function of the 1-form symmetry generators that generate a 2 -form symmetry generator cannot be described by a 2 -group, since there is no such a structure in a 2-group. Fortunately, we can find an appropriate structure by extending the 2-group to a 3-group, which we explain below.

\subsection{Global 3-group symmetry for axion electrodynamics}

Here, we review the global 3-group symmetry for the axion electrodynamics [101]. The detail of the axioms of the 3-group is explained in appendix C. Here, we summarize the ingredients of the 3 -group $\left(L \stackrel{\partial_{2}}{\rightarrow} H \stackrel{\partial_{1}}{\rightarrow} G, \triangleright,\{-,-\}\right)$ are as follows:

1. Three groups $G, H$, and $L$ : they are not necessarily Lie groups. 
2. Maps $\partial_{1}$ and $\partial_{2}$ between the three groups: $\partial_{1}: H \rightarrow G, \partial_{2}: L \rightarrow H$. These maps are group homomorphism, i.e., they are compatible with group compositions. The composition of the maps satisfies $\partial_{1} \circ \partial_{2}(l)=1_{G}$ for all $l \in L$, where $1_{G} \in G$ is the identity element in $G$.

3. Action $\triangleright$ of $G$ on $G, H, L$ by automorphism: the actions are denoted as $g \triangleright g^{\prime} \in G$, $g \triangleright h \in H$, and $g \triangleright l \in L$ for $g, g^{\prime} \in G, h \in H$, and $l \in L$. In particular the action of $G$ on $G$ is defined by conjugation: $g \triangleright g^{\prime}:=g g^{\prime} g^{-1}$. The actions are compatible with the group compositions.

4. Peiffer lifting $\{-,-\}: H \times H \rightarrow L$. In terms of the elements, the Peiffer lifting is written as $\left\{h, h^{\prime}\right\} \in L$ for $h, h^{\prime} \in H$. The action $\triangleright$ is compatible with the Peiffer lifting:

$$
g \triangleright\left\{h, h^{\prime}\right\}=\left\{g \triangleright h, g \triangleright h^{\prime}\right\} .
$$

By the discussion in section 4.1, let us specify the 3-group for the axion-photon system. First, we identify the three groups $G, H$, and $L$ as the $0-, 1-, 2$-form symmetry groups, respectively:

$$
G=\mathbb{Z}_{N}, \quad H=\mathbb{Z}_{N} \times \mathrm{U}(1), \quad L=\mathrm{U}(1) .
$$

Next, we define the maps $\partial_{1}$ and $\partial_{2}$ for the axion electrodynamics. In the correlation function, there are no maps which relate the 1-form symmetry generators to 0-form symmetry generators, or 2-form symmetry generators to 1-form symmetry generators. Therefore, we define these maps as follows,

$$
\partial_{1}\left(e^{2 \pi i m / N}, e^{i \alpha}\right)=1, \quad \partial_{2} e^{i \beta}=(1,1)
$$

for all $\left(e^{2 \pi i m / N}, e^{i \alpha}\right) \in H$ and $e^{i \beta} \in L$. Note that the requirement $\partial_{1} \circ \partial_{2}=1$ is trivially satisfied.

Third, we consider the action $\triangleright$ of $G$ on $G, H$, and $L$. Since $G=\mathbb{Z}_{N}$ is Abelian, the conjugation is trivial: $g g^{\prime} g^{-1}=g^{\prime}$. Therefore, the action of $G$ on itself is defined by a trivial one: $g \triangleright g^{\prime}=g^{\prime}$. However, the action of $G$ on $H$ should be nontrivial, since the correlation function in eq. (4.1) implies that $G$ can act on $H$. We define the action of $G$ on $H$ following the correlation function in eq. (4.1). For $e^{2 \pi i n / N} \in G=\mathbb{Z}_{N},\left(e^{2 \pi i m / N}, e^{i \alpha}\right) \in$ $H=\mathbb{Z}_{N} \times \mathrm{U}(1)$, the action $\triangleright$ is given by

$$
e^{2 \pi i n / N} \triangleright\left(e^{2 \pi i m / N}, e^{i \alpha}\right)=\left(e^{2 \pi i m / N}, e^{-2 \pi i n m / N} e^{i \alpha}\right) .
$$

Since the action of the 0 -form symmetry generator on the 2-form symmetry generator is trivial as in eq. (4.4), we define the action of $G$ on $L$ as

$$
e^{2 \pi i n / N} \triangleright e^{i \beta}=e^{i \beta} .
$$

Finally, we identify the Peiffer lifting. Since the Peiffer lifting generates an element of $L$ from the two elements of $H$, we can relate the Peiffer lifting to the correlation function 
in eq. (4.3). The diagrammatic expression in eq. (D.48) and the correlation function in eq. (4.3) suggest that we define the Peiffer lifting such that it satisfies

$$
\left\{\left(e^{2 \pi i m / N}, e^{i \alpha}\right),\left(e^{2 \pi i m^{\prime} / N}, e^{i \alpha^{\prime}}\right)\right\}\left\{\left(e^{2 \pi i m^{\prime} / N}, e^{i \alpha^{\prime}}\right),\left(e^{2 \pi i m / N}, e^{i \alpha}\right)\right\}=e^{2 \pi i m m^{\prime} / N} \in L .
$$

Since $L=\mathrm{U}(1)$ is Abelian and the right-hand side is symmetric under $m \leftrightarrow m^{\prime}$, we may introduce the Peiffer lifting as ${ }^{2}$

$$
\left\{\left(e^{2 \pi i m / N}, e^{i \alpha}\right),\left(e^{2 \pi i m^{\prime} / N}, e^{i \alpha^{\prime}}\right)\right\}=e^{2 \pi i m m^{\prime} / 2 N} .
$$

Although this definition has ambiguities under the shift $m \rightarrow m+N$ and $m^{\prime} \rightarrow m^{\prime}+N$, eq. (4.10) is unambiguous. As we discuss later, we can show that the gauge transformation law and field strength of the 3 -form gauge field in the 3-group gauge theory match the one obtained by the background gauging procedure in eq. (3.23) by this definition. ${ }^{3}$

We have defined the three groups, actions, and Peiffer lifting. We should confirm that these satisfy the axioms of the 3-group, summarized in C.1. In particular, it is nontrivial is to confirm the compatibility between the action and Peiffer lifting in eq. (4.5). The right-hand side of eq. (4.5) can be evaluated as

$$
\begin{aligned}
\left\{e^{2 \pi i n / N} \triangleright\left(e^{2 \pi i m / N}, e^{i \alpha}\right), e^{2 \pi i n / N} \triangleright\left(e^{2 \pi i m^{\prime} / N}, e^{i \alpha^{\prime}}\right)\right\} \\
\quad=\left\{\left(e^{2 \pi i m / N}, e^{-2 \pi i n m / N} e^{i \alpha}\right),\left(e^{2 \pi i m^{\prime} / N}, e^{-2 \pi i n m^{\prime} / N} e^{i \alpha^{\prime}}\right)\right\} \\
\quad=e^{2 \pi i m m^{\prime} / 2 N} .
\end{aligned}
$$

Meanwhile, the left-hand side of eq. (4.5) is

$$
e^{2 \pi i n / N} \triangleright e^{2 \pi i m m^{\prime} / 2 N}=e^{2 \pi i m m^{\prime} / 2 N},
$$

where we have used eq. (4.9). Therefore, we have confirmed that the compatibility in eq. (4.5) is satisfied.

We can understand that the correlation functions between the symmetry generators in eqs. (4.1)-(4.4) as a 3-group generalization of the current algebra, which imply that a conserved current can be a source of another current. Note that the correlation functions between symmetry generators, but not between the conserved currents, are physically meaningful in our case. This is because the conserved currents $j_{\phi E}$ and $j_{a E}$ are not gauge invariant. For the detailed discussion, see appendix B.3 and the previous paper of the present authors [101].

\subsection{Gauging 3-group symmetry}

Here, we consider the gauging the 3-group symmetry in terms of the 3-group gauge theory, and show that the gauging based on the 3 -group gauge theory is consistent with the gauging which avoids the operator shifts in section 3.3.

\footnotetext{
${ }^{2}$ We choose that a different definition of the Peiffer lifting from our previous paper to be consistent with the background gauging, although the previous definition, $\left\{\left(e^{2 \pi i m / N}, e^{i \alpha}\right),\left(e^{2 \pi i m^{\prime} / N}, e^{i \alpha^{\prime}}\right)\right\}=e^{2 \pi i m m^{\prime} / N}$, is also consistent with the axiom of the 3-group [101].

${ }^{3}$ In order to define the Peiffer lifting itself in an unambiguous way, we may need to treat the spin structure explicitly. We leave this issue as future work.
} 
In order to establish the 3 -group gauge theory, we need the Lie algebra of the 3 -group in the axion electrodynamics. The Lie algebra of $\mathrm{U}(1)$ is $i \mathbb{R}$, but the Lie algebra of $\mathbb{Z}_{N}$ does not exist since $\mathbb{Z}_{N}$ is a discrete group. However, we can introduce a $\mathbb{Z}_{N} p$-form gauge field $\omega_{p}$ by embedding $\mathbb{Z}_{N}$ to $\mathrm{U}(1)$, in which a $p$-form gauge field satisfies the condition $N A_{p}=d A_{p-1}$ with the normalization $\int_{\Sigma_{p-1}} d A_{p-1} \in 2 \pi \mathbb{Z}$ for a $(p-1)$-dimensional subspace $\Sigma_{p-1}$.

By using $\mathbb{Z}_{N}$ gauge fields as well as $\mathrm{U}(1)$ gauge fields, we now establish the background gauging of the 3-group. The 1-, 2-, 2-, and 3-form gauge fields $A_{1}, B_{2}^{E}, B_{2}^{M}$, and $C_{3}$ denote the gauge fields of $\mathbb{Z}_{N}, \mathbb{Z}_{N} \times \mathrm{U}(1)$, and $\mathrm{U}(1)$ of the 3 -group (U(1) $\rightarrow$ $\left.\mathbb{Z}_{N} \times \mathrm{U}(1) \rightarrow \mathbb{Z}_{N}, \triangleright,\{-,-\}\right)$, respectively. Here, the gauge fields $A_{1}$ and $B_{2}^{E}$ are constrained by $N A_{1}=d A_{0}$ and $N B_{2}^{E}=d B_{1}^{E}$ for the 0 - and 1-form gauge fields $A_{0}$ and $B_{1}^{E}$ with proper normalization, respectively. The action $\triangleright$ and the Peiffer lifting $\{-,-\}$ determine the gauge transformation laws and field strengths. For the gauge transformation laws, they are given in eqs. (C.44)-(C.46) as

$$
\begin{aligned}
& A_{1} \rightarrow A_{1}+d \Lambda_{0}, \\
& \left(\begin{array}{c}
B_{2}^{E} \\
B_{2}^{M}
\end{array}\right) \rightarrow\left(\begin{array}{c}
B_{2}^{E} \\
B_{2}^{M}
\end{array}\right)+\left(\begin{array}{c}
d \Lambda_{1}^{E} \\
d \Lambda_{1}^{M}
\end{array}\right)-\Lambda_{0} \triangleright\left(\begin{array}{c}
B_{2}^{E} \\
B_{2}^{M}
\end{array}\right)+\left(A_{1}+d \Lambda_{0}\right) \triangleright\left(\begin{array}{c}
d \Lambda_{1}^{E} \\
d \Lambda_{1}^{M}
\end{array}\right) \\
& =\left(\begin{array}{c}
B_{2}^{E}+d \Lambda_{1}^{E} \\
B_{2}^{M}+d \Lambda_{1}^{M}+\frac{N}{2 \pi} \Lambda_{0} B_{2}^{E}-\frac{N}{2 \pi}\left(A_{1}+d \Lambda_{0}\right) \wedge \Lambda_{1}^{E}
\end{array}\right), \\
& C_{3} \rightarrow C_{3}+d \Lambda_{2}-\left\{B_{2}^{E}+d \Lambda_{1}^{E}, \Lambda_{1}^{E}\right\}-\left\{\Lambda_{1}^{E}, B_{2}^{E}\right\} \\
& =C_{3}+d \Lambda_{2}-\frac{N}{2 \pi} \Lambda_{1}^{E} \wedge B_{2}^{E}-\frac{N}{4 \pi} \Lambda_{1}^{E} \wedge d \Lambda_{1}^{E} .
\end{aligned}
$$

We note that it is necessary to treat the two-form gauge fields as a pair of gauge fields $\left(B_{2}^{E}, B_{2}^{M}\right)$ because $\Lambda_{0} \triangleright\left(B_{2}^{E}, B_{2}^{M}\right)$ does not act on $B_{2}^{E}$ and $B_{2}^{M}$, independently. The field strengths are given in eqs. (C.55) $-($ C.57) as

$$
\begin{aligned}
F_{2} & =d A_{1}=0 \\
\left(\begin{array}{c}
H_{3}^{E} \\
H_{3}^{M}
\end{array}\right) & =\left(\begin{array}{c}
d B_{2}^{E} \\
d B_{2}^{M}
\end{array}\right)+A_{1} \triangleright\left(\begin{array}{c}
B_{2}^{E} \\
B_{2}^{M}
\end{array}\right)=\left(\begin{array}{c}
0 \\
d B_{2}^{M}-\frac{N}{2 \pi} A_{1} \wedge B_{2}^{E}
\end{array}\right), \\
G_{4} & =d C_{3}+\left\{B_{2}^{E}, B_{2}^{E}\right\}=d C_{3}+\frac{N}{4 \pi} B_{2}^{E} \wedge B_{2}^{E} .
\end{aligned}
$$

By the above structure of the gauge transformation laws and field strengths, the gauge fields coincide with the gauging that avoids operator-valued shifts.

The gauge fields $\left(A_{1}, B_{2}^{E}, B_{2}^{M}, C_{3}\right)$ correspond to $\left(A_{1}^{\phi E}, B_{2}^{a E}, B_{2}^{a M}, C_{3}^{\phi M}\right)$ given in section 3.3, since the gauge transformation laws and field strengths coincide with each other. Therefore, we have confirmed that the higher-form symmetries of the axion electrodynamics possess the 3-group structure in the viewpoint of the background gauging of the 3-group symmetry. We also have the same gauged action as the one in eq. (3.34): the coupling of $A_{1}$ and $B_{2}^{E}$ with the axion and photon are give by the combinations $d \phi-A_{1}$ and $d a-B_{2}^{E}$, respectively. Furthermore, the coupling of $B_{2}^{M}$ and $C_{3}$ with $d a-B_{2}$ and $d a-B_{2}^{E}$ are 
described as five dimensional actions, $\frac{1}{2 \pi} \int_{X_{5}}\left(d a-B_{2}\right) \wedge H_{3}$ and $-\frac{1}{2 \pi} \int_{X_{5}}\left(d \phi-A_{1}\right) \wedge G_{4}$, respectively. Since this gauging procedure gives the same action, the 't Hooft anomalies of the higher-form symmetries is also the same.

One comment is in order. As we have seen in section 3.1.2, the gauge invariance of the background fields corresponds to the invariance under the topological deformations, i.e., the conservation laws of the conserved currents. The deformation of the gauge transformation laws corresponds to the deformation of the conservation laws of the conserved currents. In our case, the deformation of the gauge transformation laws can be understood by the correlation functions of the symmetry generators in eqs. (4.1)-(4.4), which are generalizations of the ordinary current algebra. For example, the correlation function in eq. (4.1) implies that the conservation law of $j_{\phi E}$ in the presence of another current $j_{a E}$ is deformed. Note that the conserved currents $j_{\phi E}$ and $j_{a E}$ themselves are not physical observable, and we have discussed the correlation functions of the gauge invariant symmetry generators.

\section{Summary and discussion}

In this paper, we have studied the higher group structure of the higher-form symmetries and 't Hooft anomalies in the $(3+1)$-dimensional axion electrodynamics in detail by using the background gauging. We have found that the axion electrodynamics offers a simple model exhibiting the 3 -group structure and 't Hooft anomalies of the 3-group.

We have discussed two independent gauging procedures. One is to formulate the gauged action where the background gauge fields are coupled with the symmetry generators. This procedure does not rely on the existence of a 3-group structure. We have determined the gauge transformation laws and gauge invariant field strengths by the requirement that the gauged action preserves the gauge invariance for the dynamical fields. The other is the gauging based on the 3 -group gauge theories. This gauging can be established by using a global 3-group structure in ref. [101] and a mathematical procedure in the 3-group gauge theory. By comparing the gauge transformation laws and field strengths of the background gauge fields, we have then shown that the above two independent gauging procedures give the same result. Furthermore, we have determined the 't Hooft anomalies of the global 3-group symmetry. In particular, we have found a 2-group anomaly, which forbids a simultaneous gauging of the $\mathbb{Z}_{N}$ 0-form and electric $\mathbb{Z}_{N}$ 1-form symmetries.

There are several avenues for future work. One is to analyze physics in the background magnetic field, spatially varying axion field, and so on. It has been shown that in non-trivial backgrounds, mass spectra of the axion and photon are deformed $[26,54,55,116]$. We may understand such deformations of the phase structure by using higher-form symmetries, 3group, and their 't Hooft anomalies (see e.g., ref. [117] for recent discussion). Another important direction is to discuss what happens for higher-form symmetries when the axion becomes massive, while in this work we have assumed that the axion is massless. When the axion becomes massive by non-perturbative effects, there can be axionic domain walls, which have a topological domain wall charge. In the absence of the photon, it has been shown that there can be a discrete 3 -form symmetry whose charged object is a worldvolume of the axionic domain wall [118]. Therefore, the higher-group structure may be deformed in the presence of the 3 -form symmetry. 


\section{Acknowledgments}

R. Y. thanks Yuji Hirono, Taro Kimura, and Naoki Yamamoto for discussions. This work is supported in part by Japan Society of Promotion of Science (JSPS) Grant-inAid for Scientific Research (KAKENHI Grants No. 17H06462, 18H01211 (Y. H.) and $18 \mathrm{H} 01217$ (M. N.)).

\section{A 't Hooft loop and worldsheet of axionic string}

Here, we summarize the expressions of the 't Hooft loop and the worldsheet of the axionic string in terms of local fields by using dual transformations. The 't Hooft loop and the worldsheet of the axionic string can be expressed as line and surface integrals of a 1-form and a 2-form gauge fields, which are dual of $a$ and $\phi$, respectively.

\section{A.1 't Hooft loop}

First, we express the 't Hooft loop in terms of local fields. In the original formulation based on $a$ and $\phi$, the configuration of the 't Hooft loop $T\left(q_{a M}, \mathcal{C}\right)$ can be expressed as a singular part of $a$ [119]. We decompose $a$ into the singular part $a_{\mathrm{S}}$ and the regular part $a_{\mathrm{R}}$ as

$$
a=a_{\mathrm{R}}+a_{\mathrm{S}}
$$

Here, we require the configuration of the $a$ due to the monopole is expressed by $a_{\mathrm{S}}$ :

$$
\int_{\mathcal{S}} d a=\int_{\mathcal{S}} d\left(a_{\mathrm{R}}+a_{\mathrm{S}}\right)=\int_{\mathcal{S}} d a_{\mathrm{S}}=2 \pi q_{a M} \operatorname{Link}(\mathcal{S}, \mathcal{C}) .
$$

The singular part $a_{\mathrm{S}}$ can be understood as the 1-form that breaks the Bianchi identity,

$$
d d a=d d a_{\mathrm{S}}=2 \pi q_{a M} \delta_{3}(\mathcal{C}),
$$

which represents the existence of the magnetic monopole current. In the path integral formalism, we can express the 't Hooft loop as

$$
\left\langle T\left(q_{a M}, \mathcal{C}\right)\right\rangle=\int \mathcal{D}\left[\phi, a_{\mathrm{R}}\right] e^{i S\left[\phi, a_{\mathrm{R}}+a_{\mathrm{S}}\right]} .
$$

Now, we consider the explicit form of the 't Hooft loop in terms of a local field. This can be done by the dual transformation of the photon $a$ [120]. We can rewrite eq. (A.4) by using the Fourier transformation,

$$
\left\langle T\left(q_{a M}, \mathcal{C}\right)\right\rangle=\int \mathcal{D}\left[\phi, a_{\mathrm{R}}, f^{\prime}, g\right] e^{i S_{a, 1 \mathrm{st}}\left[\phi, a_{\mathrm{R}}+a_{\mathrm{S}}, f^{\prime}, g\right]},
$$

where $S_{a, 1 \text { st }}\left[\phi, a_{\mathrm{R}}+a_{\mathrm{S}}, f^{\prime}, g\right]$ is a first order derivative action for $a$ :

$$
\begin{aligned}
S_{a, 1 \mathrm{st}}= & -\frac{1}{2 e^{2}} \int_{M_{4}} f^{\prime} \wedge \star f^{\prime}-\frac{v^{2}}{2} \int_{M_{4}} d \phi \wedge \star d \phi+\frac{N}{8 \pi^{2}} \int_{M_{4}} \phi f^{\prime} \wedge f^{\prime} \\
& -\frac{1}{2 \pi} \int_{M_{4}} g \wedge\left(f^{\prime}-d a_{\mathrm{R}}-d a_{\mathrm{S}}\right) .
\end{aligned}
$$


Here, we have introduced new dynamical valuable $f^{\prime}$ and $g$, which are 2-form fields independent of $a$ and $\phi$.

We can go back to the original action in eq. (2.1) by integrating out $g$ and $f^{\prime}$, where the integral of $g$ gives us the delta function $\delta\left[f^{\prime}-d\left(a_{\mathrm{R}}+a_{\mathrm{S}}\right)\right]$. Instead, we can go to the dual action as follows. By integrating out $a_{\mathrm{R}}$, we have the delta function $\delta[d g]$, which implies that $g$ can be locally given by a 1 -form gauge field $w$,

$$
g=d w,
$$

with a gauge transformation by a 0 -form gauge parameter $\lambda_{w}$,

$$
w \rightarrow w+d \lambda_{w}
$$

Therefore, $\left\langle T\left(q_{a M}, \mathcal{C}\right)\right\rangle$ can be written as

$$
\left\langle T\left(q_{a M}, \mathcal{C}\right)\right\rangle=\int \mathcal{D}\left[\phi, v, f^{\prime}\right] e^{i\left(-\frac{1}{2 e^{2}} \int_{M_{4}} f^{\prime} \wedge \star f^{\prime}-\frac{v^{2}}{2} \int_{M_{4}} d \phi \wedge \star d \phi+\frac{N}{8 \pi^{2}} \int_{M_{4}} \phi f^{\prime} \wedge f^{\prime}-\frac{1}{2 \pi} \int_{M_{4}} d w \wedge\left(f^{\prime}-d a_{S}\right)\right)} .
$$

We find that the term given by the singular part, $e^{-\frac{i}{2 \pi} \int d w \wedge d a_{S}}$, can be expressed by a line integral of $w$ along $\mathcal{C}$ :

$$
e^{-\frac{i}{2 \pi} \int_{M_{4}} d w \wedge d a_{\mathrm{S}}}=e^{i q_{a M} \int_{M_{4}} w \wedge \delta_{3}(\mathcal{C})}=e^{i q_{a M} \int_{\mathcal{C}} w},
$$

where we have used eq. (A.3) and then eq. (3.10). This is the expression of the desired 't Hooft loop in terms of the local field.

\section{A.2 Worldsheet of axionic string}

Similarly, we can express the worldsheet of the axionic string in terms of a local field. In the original formulation, the configuration of $V\left(q_{\phi M}, \mathcal{S}\right)$ can be expressed as a singular part of $\phi$. We again decompose $\phi$ into the singular part $\phi_{\mathrm{S}}$ and the regular part $\phi_{\mathrm{R}}$ as

$$
\phi=\phi_{\mathrm{R}}+\phi_{\mathrm{S}},
$$

where we have assumed that $\phi_{\mathrm{S}}$ have non-trivial winding number,

$$
\int_{\mathcal{C}} d \phi=\int_{\mathcal{C}} d\left(\phi_{\mathrm{R}}+\phi_{\mathrm{S}}\right)=\int_{\mathcal{C}} d \phi_{\mathrm{S}}=2 \pi q_{\phi M} \operatorname{Link}(\mathcal{C}, \mathcal{S}) .
$$

The singular part $\phi_{\mathrm{S}}$ can be understood as the function that breaks the Bianchi identity of the axion,

$$
d d \phi=d d \phi_{\mathrm{S}}=2 \pi q_{\phi M} \delta_{2}(\mathcal{S}) .
$$

In the path integral formalism, we can express $V\left(q_{\phi M}, \mathcal{S}\right)$ as

$$
\left\langle V\left(q_{\phi M}, \mathcal{S}\right)\right\rangle=\int \mathcal{D}\left[\phi_{\mathrm{R}}, a\right] e^{i S\left[\phi_{\mathrm{R}}+\phi_{\mathrm{S}}, a\right]} .
$$

Now, we consider an alternative expression of $V\left(q_{\phi M}, \mathcal{S}\right)$ by the dual transformation of the axion $\phi$ to a 2-form gauge field [121]. We can again rewrite eq. (A.14) by using the Fourier transformation,

$$
\left\langle V\left(q_{\phi M}, \mathcal{S}\right)\right\rangle=\int \mathcal{D}\left[\phi, a_{\mathrm{R}}, \zeta, h\right] e^{i S_{\phi, 1 \mathrm{st}}\left[\phi_{\mathrm{R}}+\phi_{\mathrm{S}}, a, \zeta, h\right]} .
$$


Here, $S_{\phi, 1 \mathrm{st}}\left[\phi_{\mathrm{R}}+\phi_{\mathrm{S}}, a, \zeta, h\right]$ is a first order derivative action for $\phi$ :

$$
\begin{aligned}
S_{\phi, 1 \mathrm{st}}= & -\frac{1}{2 e^{2}} \int_{M_{4}} d a \wedge \star d a-\frac{v^{2}}{2} \int_{M_{4}} \zeta \wedge \star \zeta-\frac{N}{8 \pi^{2}} \int_{M_{4}} \zeta \wedge a \wedge f \\
& +\frac{1}{2 \pi} \int_{M_{4}} h \wedge\left(\zeta-d \phi_{\mathrm{R}}-d \phi_{\mathrm{S}}\right) .
\end{aligned}
$$

We have introduced new dynamical valuable $\zeta$ and $h$, which are 1-form and 3-form fields independent of $a$ and $\phi$. Note that the 3-form $h$ should be shifted under the gauge transformation of the 1-form field as

$$
a \rightarrow a+d \lambda, \quad h \rightarrow h-\frac{N}{4 \pi} d \lambda \wedge d a
$$

in order to make the action to be gauge invariant. As in the case of $S_{a, 1 s t}$, we can go back to the original action in eq. (2.1) by integrating out $\zeta$ and $h$, where the integral of $h$ gives us the delta function $\delta\left[\zeta-d\left(\phi_{\mathrm{R}}+\phi_{\mathrm{S}}\right)\right]$. Instead, we can again go to the dual action. By integrating out $\phi_{\mathrm{R}}$, we have the delta function $\delta[d h]$ implying that $h$ can be locally given by a 2 -form gauge field $b$,

$$
h=d b,
$$

with a gauge transformation by a 1 -form gauge parameter $\lambda_{b}$,

$$
b \rightarrow b+d \lambda_{b}
$$

in addition to the one corresponding to eq. (A.17),

$$
b \rightarrow b-\frac{N}{4 \pi} \lambda d a .
$$

Substituting the condition $h=d b,\left\langle V\left(q_{\phi M}, \mathcal{S}\right)\right\rangle$ can be written as

$$
\left\langle V\left(q_{\phi M}, \mathcal{S}\right)\right\rangle=\int \mathcal{D}[b, a, \zeta] e^{i\left(-\frac{1}{2 e^{2}} \int_{M_{4}} d a \wedge \star d a-\frac{v^{2}}{2} \int_{M_{4}} \zeta \wedge \star \zeta-\frac{N}{8 \pi^{2}} \int_{M_{4}} \zeta \wedge a \wedge f+\frac{1}{2 \pi} \int_{M_{4}} d b \wedge\left(\zeta-d \phi_{\mathrm{S}}\right)\right)} .
$$

We find that the term given by the singular part, $e^{-\frac{i}{2 \pi} \int_{M_{4}} d b \wedge d \phi_{\mathrm{S}}}$, can be expressed by a surface integral of $b$ along $\mathcal{S}$ :

$$
e^{-\frac{i}{2 \pi} \int_{M_{4}} d b \wedge d \phi_{\mathrm{S}}}=e^{i q_{a M} \int_{M_{4}} b \wedge \delta_{2}(\mathcal{S})}=e^{i q_{a M} \int_{\mathcal{S}} b}
$$

where we have again used eq. (A.13) and eq. (3.10). This is the expression of the worldsheet of the axionic string.

We remark that this expression of the axionic string is not invariant under eq. (A.20). Therefore, the gauge invariance for the photon seems to be violated on the axionic string. The inconsistency can be resolved by taking into account the chiral mode on the axionic string. The chiral mode charged under the U(1) gauge symmetry cancels the violation. This mechanism is nothing but the anomaly inflow mechanism [31, 32]. Note that we do not need to consider the contribution from the chiral modes in the discussion in this paper, since we focus on the bulk physics around the axionic string. 


\section{B Correlation functions}

Here, we summarize detailed calculations of the correlation functions.

\section{B.1 $\mathbb{Z}_{N}$ 0-form transformation}

We first evaluate the 0 -form transformation law in eq. (2.16). The transformation can be written by the following correlation function:

$$
\left\langle U_{\phi E}\left(e^{2 \pi i n_{\phi} / N}, \mathcal{V}\right) e^{i q_{\phi E} \phi(\mathcal{P})}\right\rangle=\mathcal{N} \int \mathcal{D}[\phi, a] e^{i S+\frac{2 \pi i n_{\phi}}{N} \int_{\mathcal{V}} j_{\phi E}+i q_{\phi E} \phi(\mathcal{P})} .
$$

Here, $\mathcal{N}$ is the normalization factor such that $\langle 1\rangle=1$. In order to evaluate the correlation function, we rewrite the point operator and symmetry generator in term of spacetime integral by using delta-function forms. The point operator $e^{i q_{\phi E} \phi(\mathcal{P})}$ can be rewritten as

$$
e^{i q_{\phi E} \phi(\mathcal{P})}=e^{i q_{\phi E} \int_{M_{4}} \phi \wedge \delta_{4}(\mathcal{P})} .
$$

Here, the delta-function form is defined in eq. (3.10). Suppose $\mathcal{V}$ can be expressed as the boundary of a 4-dimensional subspace $\Omega \mathcal{V}$. In general, it may not be taken as a boundary, but it is necessary for discussing the transformation law. The symmetry generator can be rewritten as

$$
\int_{\mathcal{V}} j_{\phi E}=\int_{\partial \Omega_{\mathcal{V}}} j_{\phi E}=\int_{\Omega_{\mathcal{V}}} d j_{\phi E}=\int_{M_{4}} d j_{\phi E} \delta_{0}\left(\Omega_{\mathcal{V}}\right)
$$

We can eliminate the symmetry generator by using

$$
\begin{aligned}
S[\phi, a]+ & \frac{2 \pi n_{\phi}}{N} \int_{M_{4}} d j_{\phi E} \delta_{0}(\Omega \mathcal{V}) \\
& =S\left[\phi-\frac{2 \pi n_{\phi}}{N} \delta_{0}\left(\Omega_{\mathcal{V}}\right), a\right]+\frac{v^{2}}{2}\left(\frac{2 \pi n_{\phi}}{N}\right)^{2} \int_{M_{4}} \delta_{1}(\mathcal{V}) \wedge \star \delta_{1}(\mathcal{V}),
\end{aligned}
$$

and the redefinition $\phi-\frac{2 \pi n_{\phi}}{N} \delta_{0}(\Omega \mathcal{V}) \rightarrow \phi$ as

$$
\left\langle U_{\phi E}\left(e^{2 \pi i n_{\phi} / N}, \mathcal{V}\right) e^{i q_{\phi E} \phi(\mathcal{P})}\right\rangle=e^{\frac{2 \pi i q_{\phi E} n_{\phi}}{N} \int_{M_{4}} \delta_{0}\left(\Omega_{\mathcal{V}}\right) \delta_{4}(\mathcal{P})}\left\langle e^{i q_{\phi E} \phi(\mathcal{P})}\right\rangle
$$

Here, we have regularized the trivial divergence $\int_{M_{4}} \delta_{1}(\mathcal{V}) \wedge \star \delta_{1}(\mathcal{V})$ by adding a local counter term. The integral $\int_{M_{4}} \delta_{0}(\Omega \mathcal{V}) \delta_{4}(\mathcal{P})$ in eq. (B.5) is the intersection number of $\Omega \mathcal{V}$ and $\mathcal{P}$, which is equal to the linking number of $\mathcal{V}$ and $\mathcal{P}$,

$$
\int_{M_{4}} \delta_{0}\left(\Omega_{\mathcal{V}}\right) \delta_{4}(\mathcal{P})=\operatorname{Link}(\mathcal{V}, \mathcal{P}) \in \mathbb{Z}
$$

Therefore we have

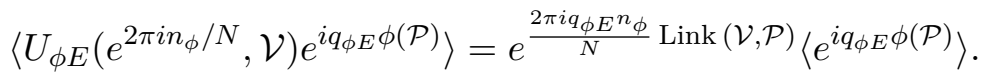




\section{B.2 $\mathbb{Z}_{N}$ 1-form transformation}

Second, we consider the correlation function, which represents the $\mathbb{Z}_{N}$ electric 1-form transformation:

$$
\left\langle U_{a E}\left(e^{2 \pi i n_{a} / N}, \mathcal{S}\right) e^{i q_{a} \int_{\mathcal{C}} a}\right\rangle=\int \mathcal{D}[\phi, a] e^{i S+\frac{2 \pi i n_{a}}{N} \int_{\mathcal{S}} j_{a E}+i q_{a} \int_{\mathcal{C}} a} .
$$

The Wilson loop $e^{i q_{a} \int_{\mathcal{C}} a}$ can be rewritten as

$$
e^{i q_{a} \int_{\mathcal{C}} a}=e^{i q_{a} \int_{M_{4}} a \wedge \delta_{3}(\mathcal{C})} .
$$

Similarly, the symmetry generator can be rewritten as

$$
\int_{\mathcal{S}} j_{a E}=\int_{\partial \mathcal{V}_{\mathcal{S}}} j_{a E}=\int_{\mathcal{V}_{\mathcal{S}}} d j_{a E}=\int_{M_{4}} d j_{a E} \wedge \delta_{1}\left(\mathcal{V}_{\mathcal{S}}\right)
$$

where we have assumed that $\mathcal{S}$ can be written as the boundary of a three dimensional subspace $\mathcal{V}_{\mathcal{S}}$. We can eliminate the symmetry generator by using

$$
\begin{aligned}
S[\phi, a]+\frac{2 \pi n_{a}}{N} \int_{M_{4}} d j_{a E} \wedge \delta_{1}\left(\mathcal{V}_{\mathcal{S}}\right)= & S\left[\phi, a+\frac{2 \pi n_{a}}{N} \delta_{1}\left(\mathcal{V}_{\mathcal{S}}\right)\right] \\
& -\frac{N}{8 \pi^{2}}\left(\frac{2 \pi n_{a}}{N}\right)^{2} \int_{M_{4}} \phi \delta_{2}(\mathcal{S}) \wedge \delta_{2}(\mathcal{S}) \\
& +\frac{1}{2 e^{2}}\left(\frac{2 \pi n_{a}}{N}\right)^{2} \int_{M_{4}} \delta_{2}(\mathcal{S}) \wedge \star \delta_{2}(\mathcal{S}),
\end{aligned}
$$

and the redefinition $a+\frac{2 \pi n_{a}}{N} \delta_{1}\left(\mathcal{V}_{\mathcal{S}}\right) \rightarrow a$ as

$$
\left\langle U_{a E}\left(e^{2 \pi i n_{a} / N}, \mathcal{V}\right) e^{i q_{a} \int_{\mathcal{C}} a}\right\rangle=e^{\frac{2 \pi i q_{a} n_{a}}{N} \int_{M_{4}} \delta_{3}(\mathcal{C}) \wedge \delta_{1}\left(\mathcal{V}_{\mathcal{S}}\right)}\left\langle e^{i q_{a} \int_{\mathcal{C}} a}\right\rangle
$$

Here, we have again regularized the trivial divergence $\frac{1}{2 e^{2}}\left(\frac{2 \pi n_{a}}{N}\right)^{2} \int_{M_{4}} \delta_{2}(\mathcal{S}) \wedge \star \delta_{2}(\mathcal{S})$ by the local counter term. Furthermore, the term $\int_{M_{4}} \phi \delta_{2}(\mathcal{S}) \wedge \delta_{2}(\mathcal{S})$ in eq. (B.11) is equal to zero if we consider a closed surface without self-intersections. The integral $\int_{M_{4}} \delta_{3}(\mathcal{C}) \wedge \delta_{1}\left(\mathcal{V}_{\mathcal{S}}\right)$ in eq. (B.12) is the intersection number of $\mathcal{V}_{\mathcal{S}}$ and $\mathcal{C}$, which is equal to the linking number of $\mathcal{S}$ and $\mathcal{C}$,

$$
\int_{M_{4}} \delta_{3}(\mathcal{C}) \wedge \delta_{1}\left(\mathcal{V}_{\mathcal{S}}\right)=\int_{\mathcal{V}_{\mathcal{S}}} \delta_{3}(\mathcal{C})=\operatorname{Link}(\mathcal{S}, \mathcal{C}) \in \mathbb{Z}
$$

Therefore, we have

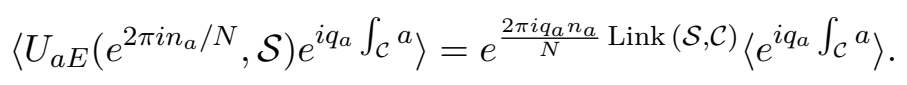

\section{B.3 Correlation functions of symmetry generators}

We here show the derivations of the correlation functions of symmetry generators. 


\section{B.3.1 Correlation function of 0 -form and 1-form symmetry generators}

First, we consider the correlation function of 0- and 1-form symmetry generators

$$
\left\langle U_{\phi E}\left(e^{2 \pi i n_{\phi} / N}, \mathcal{V}\right) U_{a E}\left(e^{2 \pi i n_{a} / N}, \mathcal{S}\right)\right\rangle=\mathcal{N} \int \mathcal{D}[\phi, a] e^{i S[\phi, a]+\frac{2 \pi i n_{\phi}}{N} \int_{\mathcal{V}} j_{\phi E}+\frac{2 \pi i n_{a}}{N} \int_{\mathcal{S}} j_{a E}},
$$

which corresponds to the Witten effect [101]. We first eliminate the 0-form symmetry generator $U_{\phi E}\left(e^{2 \pi i n_{\phi} / N}, \mathcal{V}\right)$. By the same procedures as eqs. (B.3) and (B.4), we obtain

$$
\begin{aligned}
\left\langle U_{\phi E}\left(e^{2 \pi i n_{\phi} / N}, \mathcal{V}\right) U_{a E}\left(e^{2 \pi i n_{a} / N}, \mathcal{S}\right)\right\rangle \\
=\left\langle U_{a E}\left(e^{2 \pi i n_{a} / N}, \mathcal{S}\right) e^{-\frac{i n_{\phi} n_{a}}{N} \int_{\mathcal{S}} \delta_{0}\left(\Omega_{\mathcal{V}}\right) d a}\right\rangle \\
=\left\langle U_{a E}\left(e^{2 \pi i n_{a} / N}, \mathcal{S}\right) U_{a M}\left(e^{-2 \pi i n_{\phi} n_{a} / N}, \Omega_{\mathcal{V}} \cap \mathcal{S}\right)\right\rangle .
\end{aligned}
$$

Alternatively, one can eliminate the 1-form symmetry generator as follows. Here, we assume that $\mathcal{V}$ and $\mathcal{S}$ are not intersected to each other. By using eqs. (B.10) and (B.11), we obtain

$$
\begin{aligned}
& \left\langle U_{\phi E}\left(e^{2 \pi i n_{\phi} / N}, \mathcal{V}\right) U_{a E}\left(e^{2 \pi i n_{a} / N}, \mathcal{S}\right)\right\rangle \\
& =\left\langle U_{\phi E}\left(e^{2 \pi i n_{\phi} / N}, \mathcal{V}\right) e^{\left.-\frac{n_{\phi} n_{a}}{N} \int d a \wedge \delta_{1}(\mathcal{V}) \wedge \delta_{1}\left(\mathcal{V}_{\mathcal{S}}\right)-\frac{i \pi n_{a}^{2} n_{\phi}}{N^{2}} \int \delta_{1}\left(\mathcal{V}_{\mathcal{S}}\right) \wedge d \delta_{1}\left(\mathcal{V}_{\mathcal{S}}\right) \wedge \delta_{1}(\mathcal{V})\right)}\right\rangle \\
& \quad=\left\langle U_{\phi E}\left(e^{2 \pi i n_{\phi} / N}, \mathcal{V}\right) U_{a M}\left(e^{-2 \pi i n_{\phi} n_{a} / N}, \mathcal{V} \cap \mathcal{V}_{\mathcal{S}}\right)\right\rangle e^{-\frac{i \pi n_{a}^{2} n_{\phi}}{N^{2}} \int \delta_{1}\left(\mathcal{V}_{\mathcal{S}}\right) \wedge d \delta_{1}\left(\mathcal{V}_{\mathcal{S}}\right) \wedge \delta_{1}(\mathcal{V})}
\end{aligned}
$$

The last term $e^{-\frac{i \pi n_{a}^{2} n_{\phi}}{N^{2}} \int \delta_{1}\left(\mathcal{V}_{\mathcal{S}}\right) \wedge d \delta_{1}\left(\mathcal{V}_{\mathcal{S}}\right) \wedge \delta_{1}(\mathcal{V})}$ is equal to zero, since we have assumed that $\mathcal{V}$ and $\mathcal{S}$ do not intersect. Therefore, we obtain

$$
\left\langle U_{\phi E}\left(e^{2 \pi i n_{\phi} / N}, \mathcal{V}\right) U_{a E}\left(e^{2 \pi i n_{a} / N}, \mathcal{S}\right)\right\rangle=\left\langle U_{\phi E}\left(e^{2 \pi i n_{\phi} / N}, \mathcal{V}\right) U_{a M}\left(e^{-2 \pi i n_{\phi} n_{a} / N}, \mathcal{V} \cap \mathcal{V}_{\mathcal{S}}\right)\right\rangle
$$

The correlation function implies that the EOM of the photon, i.e., the conservation law of $j_{a E}$ is deformed in the presence of the current $j_{\phi E}$. Here, the EOM of the photon corresponds to the finite redefinition which has been applied in eq. (B.22). Note that we have discussed the deformation in terms of the gauge invariant symmetry generators, since the currents $j_{\phi E}$ and $j_{a E}$ are not gauge invariant.

\section{B.3.2 Correlation function of 1-form symmetry generators}

Similarly, we can evaluate the correlation function between 1-form symmetry generators,

$$
\left\langle U_{a E}\left(e^{2 \pi i n_{a} / N}, \mathcal{S}_{1}\right) U_{a E}\left(e^{2 \pi i n_{a}^{\prime} / N}, \mathcal{S}_{2}\right)\right\rangle=\mathcal{N} \int \mathcal{D}[\phi, a] e^{i S[\phi, a]+\frac{2 \pi i n_{a}}{N} \int_{\mathcal{S}_{1}} j_{a E}+\frac{2 \pi i n_{a}^{\prime}}{N} \int_{\mathcal{S}_{2}} j_{a E}}
$$

which corresponds to the anomalous Hall effect [101]. We eliminate $U_{a E}\left(e^{2 \pi i n_{a} / N}, \mathcal{S}_{1}\right)$ by the same procedures as eqs. (B.10) and (B.11), and obtain

$$
\begin{aligned}
& \left\langle U_{a E}\left(e^{2 \pi i n_{a} / N}, \mathcal{S}_{1}\right) U_{a E}\left(e^{2 \pi i n_{a}^{\prime} / N}, \mathcal{S}_{2}\right)\right\rangle \\
& =\left\langle U_{a E}\left(e^{2 \pi i n_{a}^{\prime} / N}, \mathcal{S}_{2}\right) e^{-\frac{i n_{a}^{\prime} n_{a}}{N} \int_{\mathcal{S}_{2}} d \phi \wedge \delta_{1}\left(\mathcal{V}_{\mathcal{S}_{1}}\right)}\right\rangle \\
& =\left\langle U_{a E}\left(e^{2 \pi i n_{a}^{\prime} / N}, \mathcal{S}_{2}\right) U_{\phi M}\left(e^{2 \pi i n_{a} n_{a}^{\prime} / N},-\mathcal{V}_{\mathcal{S}_{1}} \cap \mathcal{S}_{2}\right)\right\rangle .
\end{aligned}
$$


We can consider another property of the correlation function. Remarking the following relation,

$$
U_{a E}\left(e^{2 \pi i n_{a} / N}, \mathcal{S}_{1}\right) U_{a E}\left(e^{2 \pi i n_{a}^{\prime} / N}, \mathcal{S}_{2}\right)=e^{\frac{2 \pi i}{N} \int_{M_{4}} j_{a E} \wedge\left(n_{a} \delta_{2}\left(\mathcal{S}_{1}\right)+n_{a}^{\prime} \delta_{2}\left(\mathcal{S}_{2}\right)\right)},
$$

we can simply evaluate the 1 -form symmetry generators by the redefinition $a+\frac{2 \pi n_{a}}{N} \delta_{1}\left(\mathcal{V}_{\mathcal{S}_{1}}\right)+$ $\frac{2 \pi n_{a}^{\prime}}{N} \delta_{1}\left(\mathcal{V}_{\mathcal{S}_{2}}\right) \rightarrow a$ as

$$
\begin{aligned}
& \left\langle U_{a E}\left(e^{2 \pi i n_{a} / N}, \mathcal{S}_{1}\right) U_{a E}\left(e^{2 \pi i n_{a}^{\prime} / N}, \mathcal{S}_{2}\right)\right\rangle \\
& =\left\langle U_{\phi M}\left(e^{\frac{2 \pi i n_{a} n_{a}^{\prime}}{2 N}},-\mathcal{V}_{\mathcal{S}_{1}} \cap \mathcal{S}_{2}\right) U_{\phi M}\left(e^{\frac{2 \pi i n_{a} n_{a}^{\prime}}{2 N}},-\mathcal{V}_{\mathcal{S}_{2}} \cap \mathcal{S}_{1}\right)\right\rangle \\
& =\left\langle U_{\phi M}\left(e^{\frac{2 \pi i n_{a} n_{a}^{\prime}}{N}},-\mathcal{V}_{\mathcal{S}_{1}} \cap \mathcal{S}_{2}\right)\right\rangle .
\end{aligned}
$$

This result coincides with eq. (B.20) after eliminating $U_{a E}\left(e^{2 \pi i n_{a}^{\prime} / N}, \mathcal{S}_{2}\right)$ by a topological deformation. The second line of eq. (B.22) shows that the correlation function of two 1form symmetry generators linking with each other leads to two 2-form symmetry generators, which is consistent with the diagrammatic expression in eq. (D.48). Note that the linking of surfaces is called a surface link [122, 123].

The correlation functions in eqs. (B.20) and (B.22) again imply that the conservation law of $j_{a E}$ is deformed in the presence of another $j_{a E}$. Note again that we have discussed the deformation in terms of the gauge invariant symmetry generators.

\section{B.3.3 Correlation function of 0 -form and 2-form symmetry generators}

We also show the correlation function of the 0 -form and 2-form symmetry generators:

$$
\left\langle U_{\phi E}\left(e^{2 \pi i n_{\phi} / N}, \mathcal{V}\right) U_{\phi M}\left(e^{i \alpha_{\phi}}, \mathcal{C}\right)\right\rangle=\mathcal{N} \int \mathcal{D}[\phi, a] e^{i S+\frac{2 \pi i n_{\phi}}{N} \int_{\mathcal{V}} j_{\phi E}+\frac{i \alpha_{\phi}}{2 \pi} \int_{\mathcal{C}} d \phi}
$$

The redefinition $\phi-\frac{2 \pi n_{\phi}}{N} \delta_{0}\left(\Omega_{\mathcal{V}}\right) \rightarrow \phi$ leads to

$$
\left\langle U_{\phi E}\left(e^{2 \pi i n_{\phi} / N}, \mathcal{V}\right) U_{\phi M}\left(e^{i \alpha_{\phi}}, \mathcal{C}\right)\right\rangle=\left\langle U_{\phi M}\left(e^{i \alpha_{\phi}}, \mathcal{C}\right)\right\rangle e^{-\frac{i \alpha_{\phi}}{2 \pi} \frac{2 \pi n_{\phi}}{N}} \int_{\mathcal{C}} \delta_{1}(\mathcal{V}) .
$$

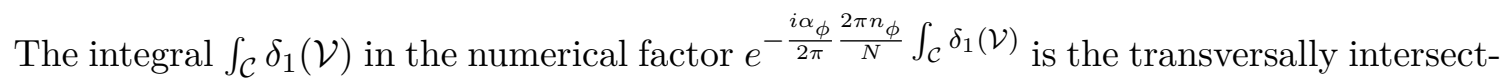
ing number of $\mathcal{C}$ and $\mathcal{V}$, which is equal to zero since both of $\mathcal{C}$ and $\mathcal{V}$ are closed. Therefore, we obtain

$$
\left\langle U_{\phi E}\left(e^{2 \pi i n_{\phi} / N}, \mathcal{V}\right) U_{\phi M}\left(e^{i \alpha_{\phi}}, \mathcal{C}\right)\right\rangle=\left\langle U_{\phi M}\left(e^{i \alpha_{\phi}}, \mathcal{C}\right)\right\rangle .
$$

Note that the resulting correlation function implies that the EOM of the axion, i.e., the conservation of $j_{\phi E}$ is not deformed in the presence of $U_{\phi M}\left(e^{i \alpha_{\phi}}, \mathcal{C}\right) .{ }^{4}$

\footnotetext{
${ }^{4}$ Technically, the correlation between $d j_{\phi E}$ and $j_{\phi M}$ is non-zero, but the correlation between $U_{\phi E}$ and $U_{\phi M}$ is zero due to the integration of $\delta_{1}(\mathcal{V})$ over $\mathcal{C}$. Since $j_{\phi E}$ is not gauge invariant, the correlation of the symmetry generators is physically meaningful.
} 


\section{3-group gauge theory}

In this section, we review the semistrict 3-group or 2-crossed module. Hereafter, we simply refer to the semistrict 3-group as the 3-group. We first present the axiom and a simple example of the 3 -group. Next, we show a Lie algebra of the 3 -group, and the 3 -group gauge theory based on it. Since the axioms of the 3-group are complicated compared to that of ordinary groups, we give a diagrammatic explanation of the axiom of the 3 -group in appendix D.

\section{C.1 Axiom of 3-group}

A 3-group $\left(L \stackrel{\partial_{2}}{\rightarrow} H \stackrel{\partial_{1}}{\rightarrow} G, \triangleright,\{-,-\}\right)$ satisfies the following axioms [102] (see also [79, 82, $83,88,124])$ :

1. $G, H$, and $L$ are groups.

2. The maps

$$
\partial_{1}: H \rightarrow G, \quad \partial_{2}: L \rightarrow H
$$

are group homomorphisms $\partial_{1}\left(h_{1} h_{2}\right)=\left(\partial_{1} h_{1}\right)\left(\partial_{1} h_{2}\right)$ and $\partial_{2}\left(l_{1} l_{2}\right)=\left(\partial_{2} l_{1}\right)\left(\partial_{2} l_{2}\right)$ for $h_{1,2} \in H$ and $l_{1,2} \in L$, respectively. They satisfy

$$
\partial_{1} \circ \partial_{2}(l)=1_{G}
$$

for all $l \in L$, where $1_{G} \in G$ is the identity element in $G$.

3. The symbol $\triangleright$ is an action of $g \in G$ on $g^{\prime} \in G, h \in H$, and $l \in L$ by automorphisms, $g \triangleright g^{\prime} \in G, g \triangleright h \in H$, and $g \triangleright l \in L$. In particular, the action $g \triangleright g^{\prime}$ is defined by conjugation,

$$
g \triangleright g^{\prime}:=g g^{\prime} g^{-1} .
$$

4. The maps $\partial_{1,2}$ are $G$-equivalent, that is, for all $g \in G, h \in H$, and $l \in L$,

$$
g \triangleright\left(\partial_{1} h\right)=\partial_{1}(g \triangleright h), \quad g \triangleright\left(\partial_{2} l\right)=\partial_{2}(g \triangleright l) .
$$

5. The Peiffer lifting $\{-,-\}$ is a map $H \times H \rightarrow L$. In terms of the elements,

$$
\left\{h_{1}, h_{2}\right\} \in L,
$$

for $h_{1,2} \in H$. The Peiffer lifting satisfies

$$
\begin{aligned}
\partial_{2}\left\{h_{1}, h_{2}\right\} & =h_{1} h_{2} h_{1}^{-1}\left(\partial_{1} h_{1}\right) \triangleright h_{2}^{-1}, \\
g \triangleright\left\{h_{1}, h_{2}\right\} & =\left\{g \triangleright h_{1}, g \triangleright h_{2}\right\}, \\
\left\{\partial_{2} l_{1}, \partial_{2} l_{2}\right\} & =l_{1} l_{2} l_{1}^{-1} l_{2}^{-1}, \\
\left\{h_{1} h_{2}, h_{3}\right\} & =\left\{h_{1}, h_{2} h_{3} h_{2}^{-1}\right\}\left(\partial_{1} h_{1}\right) \triangleright\left\{h_{2}, h_{3}\right\}, \\
\left\{h_{1}, h_{2} h_{3}\right\} & =\left\{h_{1}, h_{2}\right\}\left\{h_{1}, h_{3}\right\}\left\{\partial_{2}\left\{h_{1}, h_{3}\right\}^{-1},\left(\partial_{1} h_{1}\right) \triangleright h_{2}\right\}, \\
\left\{\partial_{2} l, h\right\}\left\{h, \partial_{2} l\right\} & =l\left(\partial_{1} h\right) \triangleright l^{-1},
\end{aligned}
$$

for $h_{1,2,3} \in H$ and $l_{1,2} \in L$. 
We note that the 3-group contains a 2-group $\left(L \stackrel{\partial_{2}}{\rightarrow} H, \triangleright^{\prime}\right)$ as the subgroup, where the actions $\triangleright^{\prime}: H \rightarrow H$ and $\triangleright^{\prime}: H \rightarrow L$ are defined as $h \triangleright^{\prime} h^{\prime}:=h h^{\prime} h^{-1}$ and $h \triangleright^{\prime} l:=l\left\{\partial_{2} l^{-1}, h\right\}$ for $h, h^{\prime} \in H$ and $l \in L$, respectively. The (strict) 2-group $\left(L \stackrel{\partial_{2}}{\rightarrow} H, \triangleright^{\prime}\right)$ is a set of two groups $H$ and $L$, a group homomorphism $\partial_{2}: L \rightarrow H$, and an action $\triangleright^{\prime}$ of $H$ on $H$ and $L$. The map $\partial_{2}$ is compatible with the action, $h \triangleright \partial_{2}(l)=\partial_{2}(h \triangleright l)$ for $h \in H$ and $l \in L$. The action of $\partial_{2}(l) \in H$ on $l^{\prime} \in L$ satisfies $\partial_{2}(l) \triangleright^{\prime} l^{\prime}=l l^{\prime} l^{-1}$, which is called the Peiffer identity. One can easily check that $\left(L \stackrel{\partial_{2}}{\rightarrow} H, \triangleright^{\prime}\right)$ satisfies these axioms. On the other hand, $\left(H \stackrel{\partial_{f}}{\rightarrow} G, \triangleright\right)$ is generally not a 2-group. From eq. (C.6), $\left(\partial_{1} h\right) \triangleright h^{\prime}=h h^{\prime} h^{-1} \partial_{2}\left\{h, h^{\prime-1}\right\}$ follows. In this sense, the Peiffer lifting measures the failure of the Peiffer identity.

\section{C.2 Example of 3-group}

Before explaining the 3-group gauge theory, we give a simple and non-trivial example of the 3 -group. It is given by an $n$-dimensional Euclidean group (or isometry group) $\operatorname{ISO}(n)$,

$$
\operatorname{ISO}(n)=\left\{\left(\begin{array}{cc}
A & \boldsymbol{a} \\
\mathbf{0}_{n}^{T} & 1
\end{array}\right) \in M(n+1, \mathbb{R}) \mid A \in \mathrm{O}(n), \boldsymbol{a} \in \mathbb{R}^{n}\right\},
$$

and we show that we can decompose the Euclidean group as a 3-group. Hereafter, we abbreviate $\mathbf{0}_{n}^{T}$ to 0 . Note that the product of two elements $h_{1}, h_{2} \in \operatorname{ISO}(n)$,

$$
h_{1}=\left(\begin{array}{cc}
A_{1} & \boldsymbol{a}_{1} \\
0 & 1
\end{array}\right), \quad h_{2}=\left(\begin{array}{cc}
A_{2} & \boldsymbol{a}_{2} \\
0 & 1
\end{array}\right)
$$

is

$$
h_{1} h_{2}=\left(\begin{array}{cc}
A_{1} A_{2} & A_{1} \boldsymbol{a}_{2}+\boldsymbol{a}_{1} \\
0 & 1
\end{array}\right) \in \operatorname{ISO}(n) .
$$

The Euclidean group can be decomposed into the orthogonal group $\mathrm{O}(n)$ and translation group $\mathbb{R}^{n}$. In other words, there are a projection map $\partial_{1}$ and an embedding map $\partial_{2}$,

$$
\mathbb{R}^{n} \stackrel{\partial_{2}}{\longrightarrow} \operatorname{ISO}(n) \stackrel{\partial_{1}}{\longrightarrow} \mathrm{O}(n) .
$$

The actions of $\partial_{2}$ and $\partial_{1}$ on $\boldsymbol{a}_{1} \in \mathbb{R}^{n}$ and $h_{2} \in \operatorname{ISO}(n)$ are

$$
\partial_{2}\left(\boldsymbol{a}_{1}\right):=\left(\begin{array}{cc}
1_{n} & \boldsymbol{a}_{1} \\
0 & 1
\end{array}\right)
$$

and

$$
\partial_{1}\left(h_{2}\right):=A_{2},
$$

respectively. These maps are compatible with products. In fact, the embedding map $\partial_{2}$ satisfies

$$
\partial_{2}\left(\boldsymbol{a}_{1}+\boldsymbol{a}_{2}\right)=\partial_{2}\left(\boldsymbol{a}_{1}\right) \partial_{2}\left(\boldsymbol{a}_{2}\right)=\partial_{2}\left(\boldsymbol{a}_{2}\right) \partial_{2}\left(\boldsymbol{a}_{1}\right),
$$

and $\partial_{1}$ satisfies

$$
\partial_{1}\left(h_{1} h_{2}\right)=\partial_{1}\left(h_{1}\right) \partial_{1}\left(h_{2}\right) .
$$

The maps $\partial_{1,2}$ satisfy

$$
\partial_{1} \circ \partial_{2}(\boldsymbol{a})=1_{n} .
$$


Note that we would decompose $\operatorname{ISO}(n)$ as $\mathrm{O}(n) \rightarrow \operatorname{ISO}(n) \rightarrow \mathbb{R}^{n}$, but this decomposition is not compatible with the product.

The elements of $\mathbb{R}^{n}$, ISO $(n)$, and $\mathrm{O}(n)$ are transformed under an action generated by $\mathrm{O}(n)$. We denote and define the action as

$$
\begin{aligned}
& A_{1} \triangleright \boldsymbol{a}_{2}:=A_{1} \boldsymbol{a}_{2}, \\
& A_{1} \triangleright h_{2}:=\left(\begin{array}{cc}
A_{1} & \mathbf{0} \\
0 & 1
\end{array}\right) h_{2}\left(\begin{array}{cc}
A_{1}^{-1} & \mathbf{0} \\
0 & 1
\end{array}\right)=\left(\begin{array}{cc}
A_{1} A_{2} A_{1}^{-1} & A_{1} \boldsymbol{a}_{2} \\
0 & 1
\end{array}\right),
\end{aligned}
$$

and

$$
A_{1} \triangleright A_{2}:=A_{1} A_{2} A_{1}^{-1} .
$$

One can check that the actions are compatible with $\partial_{1,2}$,

$$
\partial_{2}\left(A_{1} \triangleright \boldsymbol{a}_{2}\right)=A_{1} \triangleright \partial_{2}\left(\boldsymbol{a}_{2}\right),
$$

and

$$
\partial_{1}\left(A_{1} \triangleright h_{2}\right)=A_{1} \triangleright \partial_{1}\left(h_{2}\right) .
$$

Finally we determine the Peiffer lifting. Since we can obtain an element of $\mathrm{O}(n)$ from $\operatorname{ISO}(n)$ by $\partial_{1}$, we can construct an action of $\operatorname{ISO}(n)$ itself by using $\partial_{1}$ as

$$
\partial_{1}\left(h_{1}\right) \triangleright h_{2}=\left(\begin{array}{cc}
A_{1} & \mathbf{0} \\
0 & 1
\end{array}\right) h_{2}\left(\begin{array}{cc}
A_{1}^{-1} & \mathbf{0} \\
0 & 1
\end{array}\right)=\left(\begin{array}{cc}
A_{1} A_{2} A_{1}^{-1} & A_{1} \boldsymbol{a}_{2} \\
0 & 1
\end{array}\right) .
$$

On the other hand, the element of $\operatorname{ISO}(n)$ can act on itself as

$$
\begin{aligned}
h_{1} h_{2} h_{1}^{-1} & =\left(\begin{array}{cc}
A_{1} & \boldsymbol{a}_{1} \\
0 & 1
\end{array}\right)\left(\begin{array}{cc}
A_{2} & \boldsymbol{a}_{2} \\
0 & 1
\end{array}\right)\left(\begin{array}{cc}
A_{1}^{-1} & -A_{1}^{-1} \boldsymbol{a}_{1} \\
0 & 1
\end{array}\right) \\
& =\left(\begin{array}{cc}
A_{1} A_{2} A_{1}^{-1}-A_{1} A_{2} A_{1}^{-1} \boldsymbol{a}_{1}+A_{1} \boldsymbol{a}_{2}+\boldsymbol{a}_{1} \\
0 & 1
\end{array}\right) .
\end{aligned}
$$

Therefore, two actions $h_{1} h_{2} h_{1}^{-1}$ and $\partial_{1}\left(h_{1}\right) \triangleright h_{2}$ are different. The difference is the lack of $\boldsymbol{a}_{1}$ due to the projection $\partial_{1}$. We can measure the difference by

$$
h_{1} h_{2} h_{1}^{-1}\left(\partial_{1}\left(h_{1}\right) \triangleright h_{2}\right)^{-1}=\left(\begin{array}{cc}
1_{n}-A_{1} A_{2} A_{1}^{-1} \boldsymbol{a}_{1}+\boldsymbol{a}_{1} \\
0 & 1
\end{array}\right),
$$

which can be an image of $\partial_{2}$. Therefore, we can define the Peiffer lifting,

$$
\{-,-\}: \operatorname{ISO}(n) \times \operatorname{ISO}(n) \rightarrow \mathbb{R}^{n}
$$

as

$$
\left\{h_{1}, h_{2}\right\}:=-A_{1} A_{2} A_{1}^{-1} \boldsymbol{a}_{1}+\boldsymbol{a}_{1},
$$

which satisfies

$$
\partial_{2}\left(\left\{h_{1}, h_{2}\right\}\right)=h_{1} h_{2} h_{1}^{-1}\left(\partial_{1}\left(h_{1}\right) \triangleright h_{2}\right)^{-1} .
$$

One can explicitly check that these definitions satisfy other axioms of the 3-group (C.7)(C.11). 


\section{C.3 Lie algebra of 3-group}

We consider the (background) gauging of the 3-group. In order to introduce the gauge fields and their gauge transformation laws, we need the Lie algebra of the 3 -group. We denote the Lie algebra of $G, H$, and $L$ as $\mathfrak{g}, \mathfrak{h}$, and $\mathfrak{l}$, respectively. The Lie 3-group (differential 2-crossed module) is defined by the following axioms:

1. $\mathfrak{g}, \mathfrak{h}$, and $\mathfrak{l}$ are Lie algebras.

2. The maps

$$
\partial_{1}: \mathfrak{h} \rightarrow \mathfrak{g}, \quad \partial_{2}: \mathfrak{l} \rightarrow \mathfrak{h}
$$

are $\mathfrak{g}$-equivalent homomorphisms

$$
\partial_{1}\left[\underline{h}_{1}, \underline{h}_{2}\right]=\left[\partial_{1} \underline{h}_{1}, \partial_{1} \underline{h}_{2}\right], \quad \partial_{2}\left[\underline{l}_{1}, \underline{l}_{2}\right]=\left[\partial_{2} \underline{l}_{1}, \partial_{2} \underline{l}_{2}\right],
$$

for $\underline{h}_{1,2} \in \mathfrak{h}$ and $\underline{l}_{1,2} \in \mathfrak{l}$, respectively. They satisfy

$$
\partial_{1} \circ \partial_{2} \underline{l}=0
$$

3. $\triangleright$ is an action of $\underline{g} \in \mathfrak{g}$ on $\underline{g}^{\prime} \in \mathfrak{g}, \underline{h} \in \mathfrak{h}$, and $\underline{l} \in \mathfrak{l}$ by automorphisms, $\underline{g} \triangleright \underline{g}^{\prime} \in \mathfrak{g}$, $\underline{g} \triangleright \underline{h} \in \mathfrak{h}$, and $\underline{g} \triangleright \underline{\underline{l}} \in \mathfrak{l}$. The action $\underline{g} \triangleright \underline{g}^{\prime}$ is defined by the commutator,

$$
\underline{g} \triangleright \underline{g}^{\prime}:=\left[\underline{g}, \underline{g^{\prime}}\right] .
$$

4. $\partial_{1,2}$ are $\mathfrak{g}$-equivalent, that is,

$$
\underline{g} \triangleright\left(\partial_{1} \underline{h}\right)=\partial_{1}(\underline{g} \triangleright \underline{h}), \quad \underline{g} \triangleright\left(\partial_{2} \underline{l}\right)=\partial_{2}(\underline{g} \triangleright \underline{l}) .
$$

5. The Peiffer lifting $\{-,-\}$ is a map $\mathfrak{h} \times \mathfrak{h} \rightarrow \mathfrak{l}$. In terms of the elements,

$$
\left\{\underline{h}_{1}, \underline{h}_{2}\right\} \in \mathfrak{l}
$$

for $\underline{h}_{1,2} \in \mathfrak{l}$. The Peiffer lifting satisfies

$$
\begin{aligned}
\partial_{2}\left\{\underline{h}_{1}, \underline{h}_{2}\right\} & =\left[\underline{h}_{1}, \underline{h}_{2}\right]-\left(\partial_{1} \underline{h}_{1}\right) \triangleright \underline{h}_{2}, \\
\underline{g} \triangleright\left\{\underline{h}_{1}, \underline{h}_{2}\right\} & =\left\{\underline{g} \triangleright \underline{h}_{1}, \underline{h}_{2}\right\}+\left\{\underline{h}_{1}, \underline{g} \triangleright \underline{h}_{2}\right\}, \\
\left\{\partial_{2} \underline{l}_{1}, \partial_{2} \underline{l}_{2}\right\}= & {\left[\underline{l}_{1}, \underline{l}_{2}\right], } \\
\left\{\left[\underline{h}_{1}, \underline{h}_{2}\right], h_{3}\right\}= & \left\{\underline{h}_{1},\left[\underline{h}_{2}, \underline{h}_{3}\right]\right\}+\left(\partial_{1} \underline{h}_{1}\right) \triangleright\left\{\underline{h}_{2}, \underline{h}_{3}\right\} \\
& -\left\{\underline{h}_{2},\left[\underline{h}_{1}, \underline{h}_{3}\right]\right\}-\left(\partial_{1} \underline{h}_{2}\right) \triangleright\left\{\underline{h}_{1}, \underline{h}_{3}\right\}, \\
\left\{\underline{h}_{1},\left[\underline{h}_{2}, \underline{h}_{3}\right]\right\},= & \left\{\partial_{2}\left\{\underline{h}_{1}, \underline{h}_{2}\right\}, \underline{h}_{3}\right\}-\left\{\partial_{2}\left\{\underline{h}_{1}, \underline{h}_{3}\right\}, \underline{h}_{2}\right\}, \\
\left\{\partial_{2} \underline{l}_{1}, \underline{h}_{2}\right\}+\left\{\underline{h}_{2}, \partial_{2} \underline{l}_{1}\right\}= & -\left(\partial_{1} \underline{h}\right) \triangleright \underline{l}_{1},
\end{aligned}
$$

for $\underline{h}_{1,2} \in H$ and $\underline{l}_{1,2} \in L$. 


\section{C.4 3-group gauge theory}

Now, we formulate a 3-group gauge theory [79, 82, 83]. We introduce 1-, 2-, and 3-form gauge fields, $A_{1}, B_{2}$, and $C_{3}$, which are $\mathfrak{g}-, \mathfrak{h}-$, and $\mathfrak{l}$-valued differential forms, respectively. If we write the basis of the Lie algebras $\mathfrak{g}, \mathfrak{h}$, and $\mathfrak{l}$ as $\left\{u_{A}\right\},\left\{v_{a}\right\}$, and $\left\{w_{\alpha}\right\}$, respectively, the gauge fields can be written as $A_{1}=A_{1}^{A} u_{A}, B_{2}=B^{a} v_{a}$, and $C_{3}=C_{3}^{\alpha} w_{\alpha}$, respectively.

By the structure of the Lie algebra, the gauge transformation laws are given as follows. Let $g, h_{1}=h_{1}^{a} v_{a}$, and $l_{2}=l_{2}^{\alpha} w_{\alpha}$ be the $G$-, $\mathfrak{h}$-, and l-valued $0-, 1$-, and 2-form gauge parameters, respectively. Then the gauge transformations are given by

$$
\begin{aligned}
& A_{1} \rightarrow A_{1}^{\prime}=g \triangleright A_{1}+g d g^{-1}+\partial_{1} h_{1}, \\
& B_{2} \rightarrow B_{2}^{\prime}=g \triangleright B_{2}+d h_{1}-h_{1} \wedge h_{1}+A_{1}^{\prime} \triangleright h_{1}+\partial_{2} l_{2}, \\
& C_{3} \rightarrow C_{3}^{\prime}=g \triangleright C_{3}+d l_{2}+A_{1}^{\prime} \triangleright l_{2}+\left\{\partial_{2} l_{2}, h_{1}\right\}-\left\{B_{2}^{\prime}, h_{1}\right\}-\left\{h_{1}, g^{-1} \triangleright B_{2}\right\} .
\end{aligned}
$$

Here, we have used the following notations:

$$
\begin{aligned}
g \triangleright A_{1} & =A_{1}^{A} g \triangleright u_{A}=A_{1}^{A}\left(g u_{A} g^{-1}\right), \\
g \triangleright B_{2} & =B_{2}^{a}\left(g \triangleright v_{a}\right), \\
g \triangleright C_{3} & =C_{3}^{\alpha}\left(g \triangleright w_{\alpha}\right), \\
h_{1} \wedge h_{1} & =h_{1}^{a} \wedge h_{1}^{b} v_{a} v_{b}=\frac{1}{2} h_{1}^{a} \wedge h_{1}^{b}\left[v_{a}, v_{b}\right], \\
A_{1}^{\prime} \triangleright h_{1} & =\left(A_{1}^{\prime}\right)^{A} \wedge h_{1}^{a}\left(u_{A} \triangleright v_{a}\right), \\
\partial_{1} h_{1} & =h_{1}^{a}\left(\partial_{1} v_{a}\right), \\
\partial_{2} l_{2} & =l_{2}^{\alpha}\left(\partial_{2} w_{\alpha}\right), \\
\left\{B_{2}^{\prime}, h_{1}\right\} & =\left(B_{2}^{\prime}\right)^{a} \wedge h_{1}^{b}\left\{v_{a}, v_{b}\right\} .
\end{aligned}
$$

The field strengths are defined by

$$
\begin{aligned}
F & :=d A_{1}+A_{1} \wedge A_{1}, \\
H & :=d B_{2}+A_{1} \triangleright B_{2}, \\
G & :=d C_{3}+A_{1} \triangleright C_{3}+\left\{B_{2}, B_{2}\right\} .
\end{aligned}
$$

Note that the 3-group gauge theory for the axion electrodynamics can be obtained by substituting $u_{A}=-i, v_{a}=-i, w_{\alpha}=-i, g=e^{i \Lambda_{0}}, h_{1}=-i \Lambda_{1}$, and $l_{2}=-i \Lambda_{2}$.

\section{Diagrammatic expression of 3-group}

Here, we explain the 3-group diagrammatically. The definition of the 3 -group based on the axioms seems abstract, but we show that the axioms of the 3 -group can be understood in a more intuitive way. In particular, we show that all of the axioms of the 3-group can be translated to the only one simple statement "the group elements are topological" in the viewpoint of the higher-form symmetries. 


\section{D.1 Elements of groups as topological objects}

We identify the elements of groups $G, H$, and $L$ as $(D-1)-,(D-2)$-, and ( $D-3)$-dimensional topological objects respectively. By the identification, we may relate the groups $G, H$, and $L$ as symmetry groups of $0-, 1-$, and 2-form symmetries. Unlike ordinary higher-form symmetries, the topological objects may exit as the boundaries of one-dimensional higher topological objects. Hereafter, we take $D=3$ for simplicity, which is sufficient to describe all of their objects. In this case, the group elements of $G, H$, and $L$ are expressed by surfaces, lines, and points, respectively. Note that we can easily have the $D=4$ case by extending the objects such as worldvolumes, worldsurfaces, and worldlines along the fourth direction, e.g., temporal direction.

The elements $g \in G, h \in H$, and $l \in L$ can be graphically expressed as follows:

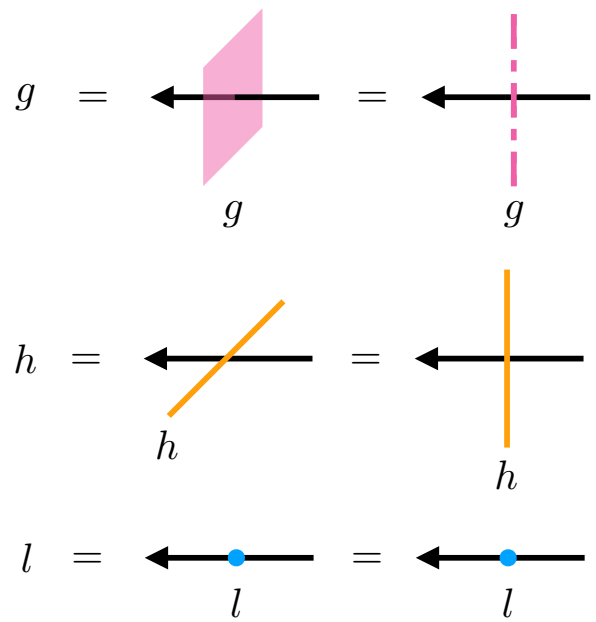

Here, the right-hand sides of the above equations are projections of the diagrams. The black left arrows represent the order of the products. We require that the elements of the groups can freely move as long as they intersect with the left arrow.

By using the left arrow, the group operations can be expressed as follows:

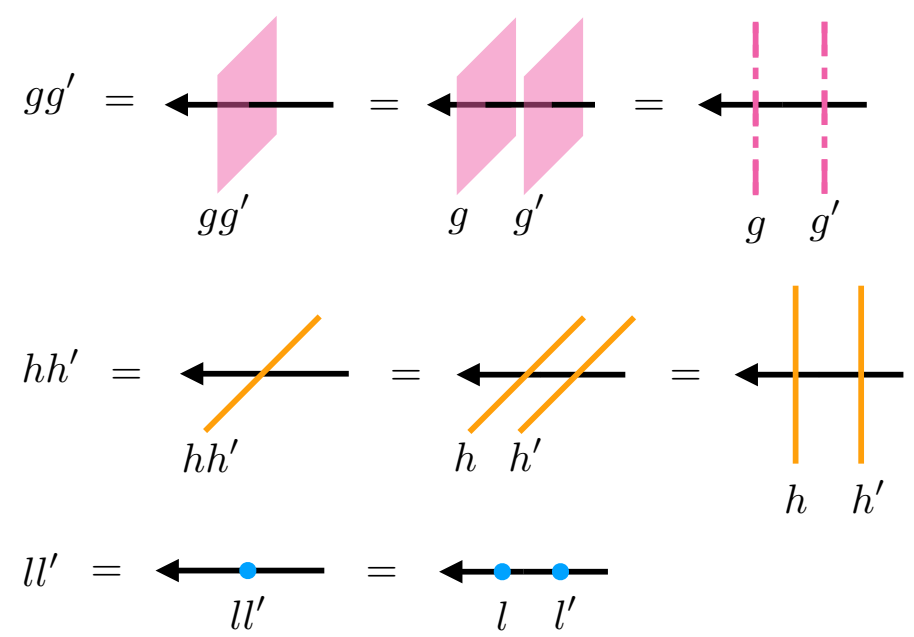

The identity elements, $1_{G} \in G, 1_{H} \in H$, and $1_{L} \in L$ are represented as a dotted surface, line, and point, respectively. Frequently, we abbreviate these identity elements to nothing. 
They are explicitly described as follows:

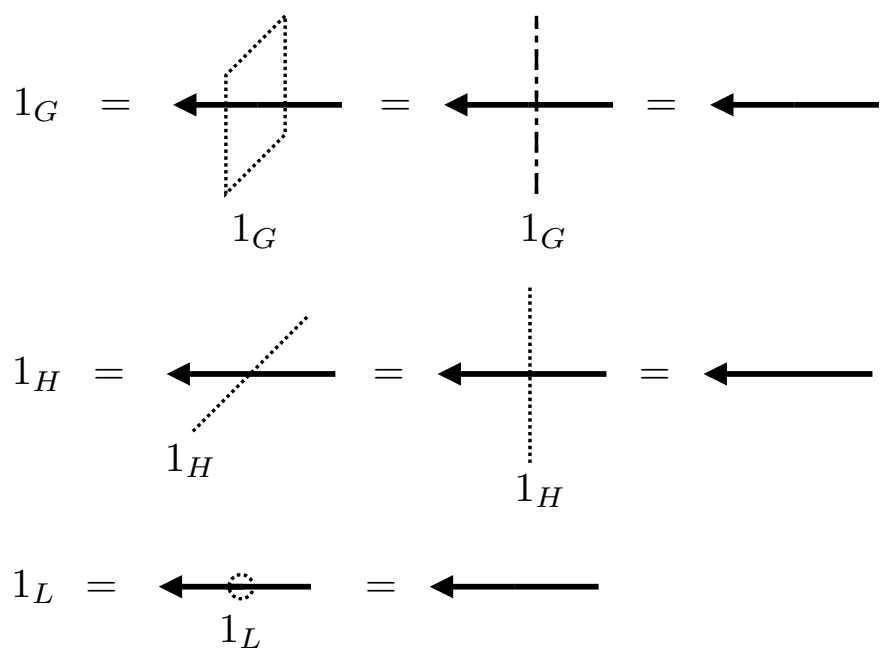

Finally, we express the inverses of the elements, $g^{-1} \in G, h^{-1} \in H$, and $l^{-1} \in L$ as objects which annihilate $g, h$ and $l$, respectively. One of the properties of the inverses is that we can connect the object $g$ and $h$ with the inverses $g^{-1}$ and $h^{-1}$ as intermediate states of the annihilation, respectively:

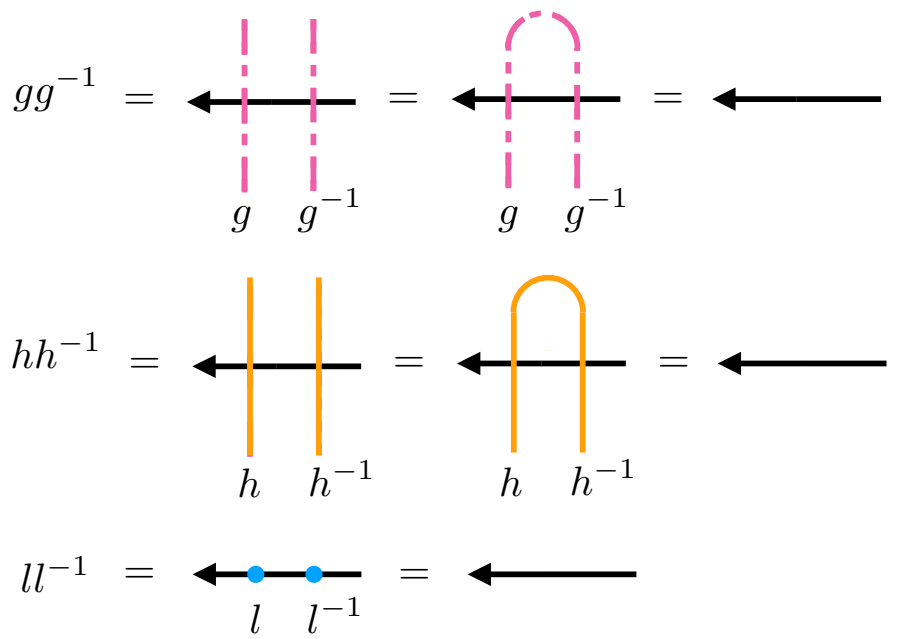

\section{D.2 $\partial_{1}$ and $\partial_{2}$ : taking interior of topological objects}

Next, we consider diagrammatic expression of the maps $\partial_{1,2}$. Since we have regarded the elements of the groups as generally extended objects, the elements can be boundaries of the other objects. The maps $\partial_{1,2}$ give the elements of the interior from the boundary elements:
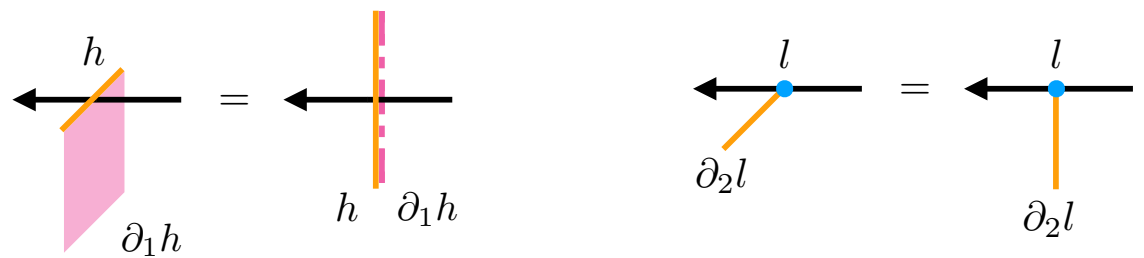
The right-hand sides of the above equations are projected diagrams. By the expression, the axiom $\partial_{1} \circ \partial_{2} l=1_{G}$ in eq. (C.20) is manifest, since an interior of an interior is nothing (conversely, the boundary of a boundary is nothing). The property of group homomorphism is just saying that the product of the elements is compatible with the product of the interior of the elements:

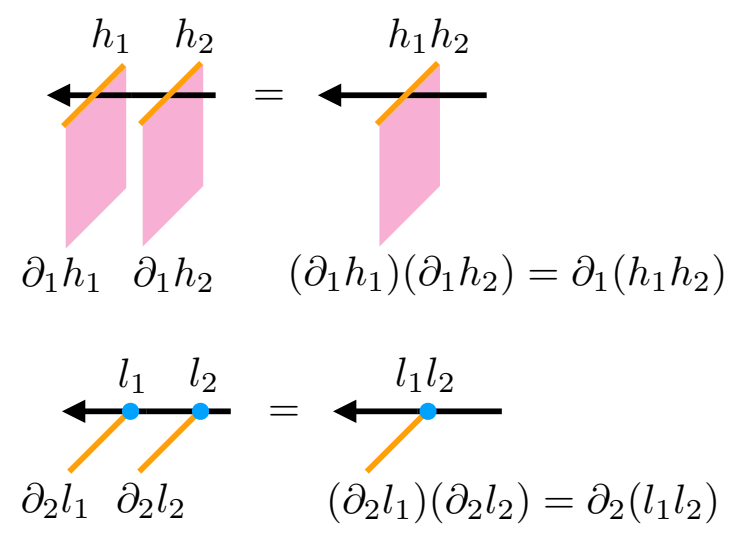

While the elements should intersect with the left arrow, we allow $l \in L$ to move vertically as long as $\partial_{2} l \in H$ intersects with the left arrow:

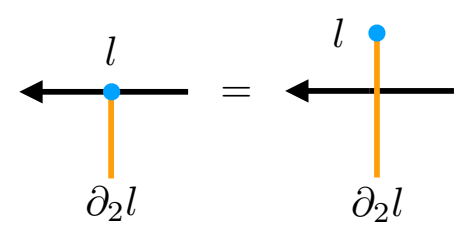

This property implies that $L$ and $H$ have a 2-group structure, which we use in section D.5. As an application, we can deform $l$ and $l^{-1}$ as follows:

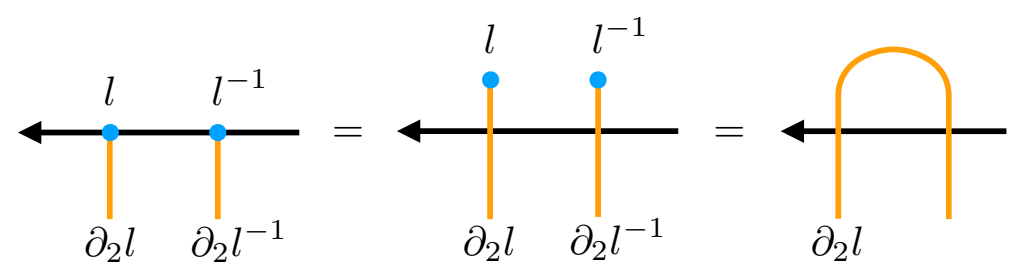

\section{D.3 Action of $G$ : enclosing elements by surfaces}

Third, we express the actions of $G$ on $G, H$, and $L$, following the above diagrammatic expressions. The action $\triangleright$ of $g \in G$ on $g^{\prime} \in G, h \in H$ and $l \in L$ can be simply described as the enclosing by $g$ and $g^{-1}$ :

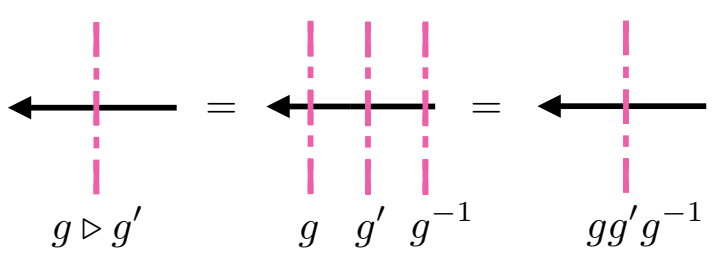




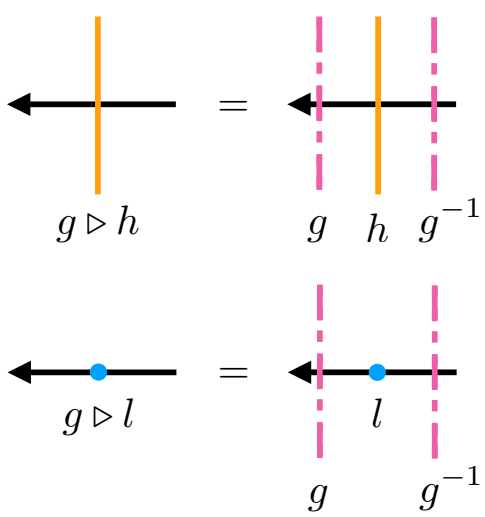

In particular, the axiom $g \triangleright g^{\prime}=g g^{\prime} g^{-1}$ given in eq. (C.3) is manifest in our diagram. Furthermore, the $G$-equivalence of $\partial_{1,2}$ in eq. (C.4) can be simply understood as the compatibility of $\partial_{1,2}$ with the action:

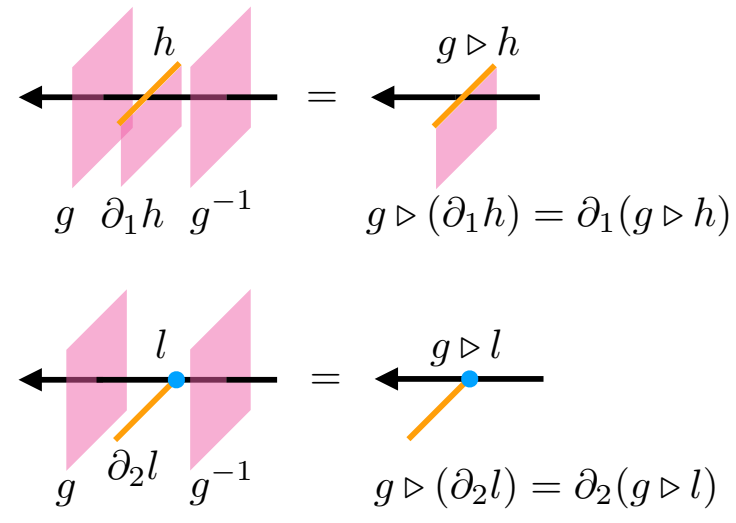

\section{D.4 Peiffer lifting: braiding of elements of $H$}

Finally, we express the Peiffer lifting diagrammatically. We determine the expression of it as a braid of two elements in $H$ such that the axiom in eq. (C.6) is satisfied:

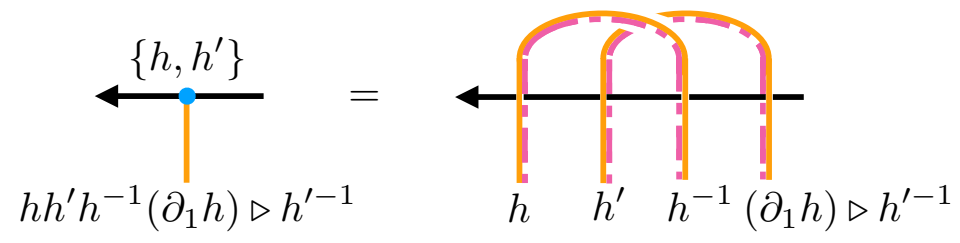

In the right-hand side, the line of $h$ braids with the line of $h^{\prime}$. Since $h^{\prime}$ intersects with $\partial_{1} h$, the surface of $\partial_{1} h$ acts on the line $h^{\prime}$. Therefore, the line of $h^{\prime}$ ends on $\left(\partial_{1} h\right) \triangleright h^{-1}=$ $\left(\left(\partial_{1} h\right) \triangleright h^{\prime}\right)^{-1}$. For the relation between the 3 -group and braids, see, e.g., refs. [125, 126].

While we have expressed the Peiffer lifting diagrammatically, it is non-trivial whether the other axioms are satisfied in terms of the diagram or not. We confirm that our diagram of the Peiffer lifting satisfies all of the axioms of the Peiffer lifting as follows: 
- Equation (C.7):

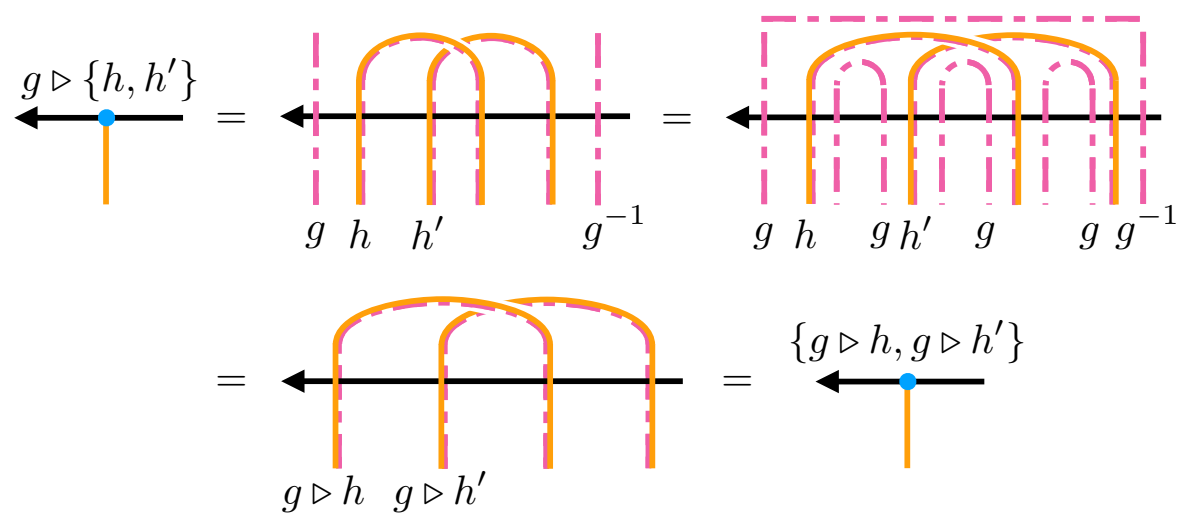

Here, we have used the property in eq. (D.10) for the third expression.

- Equation (C.8):

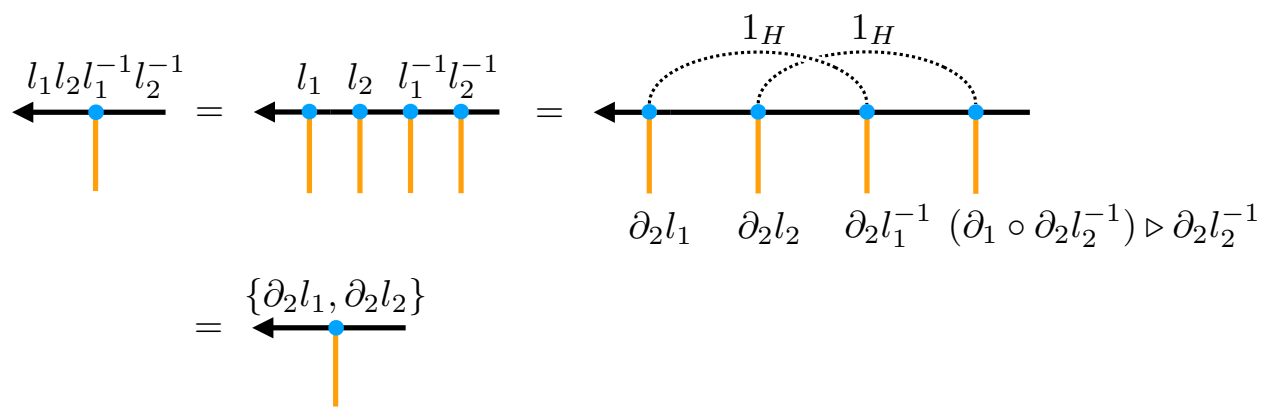

Here, we have used $\partial_{2} l_{2}^{-1}=\left(\partial_{1} \circ \partial_{2} l_{1}\right) \triangleright \partial_{2} l_{2}^{-1}$, since $\partial_{1} \circ \partial_{2} l_{1}=1_{G}$.

- Equation (C.9):

$$
\begin{aligned}
& \left(h_{1} h_{2}\right) h_{3}\left(h_{1} h_{2}\right)^{-1} \\
& \times\left(\partial_{1}\left(h_{1} h_{2}\right)\right) \triangleright h_{3}^{-1}
\end{aligned}=\underset{\left(h_{1} h_{2}\right) h_{3}\left(h_{1} h_{2}\right)^{-1} \quad\left(\partial_{1}\left(h_{1} h_{2}\right)\right) \triangleright h_{3}^{-1}}{\left\{h_{1} h_{2}, h_{3}\right\}}
$$

Here, we have used $\left(\partial_{1}\left(h_{1} h_{2}\right)\right) \triangleright h_{3}^{-1}=\left(\left(\partial_{1} h_{1}\right)\left(\partial_{2} h_{2}\right)\right) \triangleright h_{3}^{-1}=\left(\partial_{1} h_{1}\right) \triangleright\left(\partial_{2} h_{2}\right) \triangleright h_{3}^{-1}$. 
- Equation (C.10):

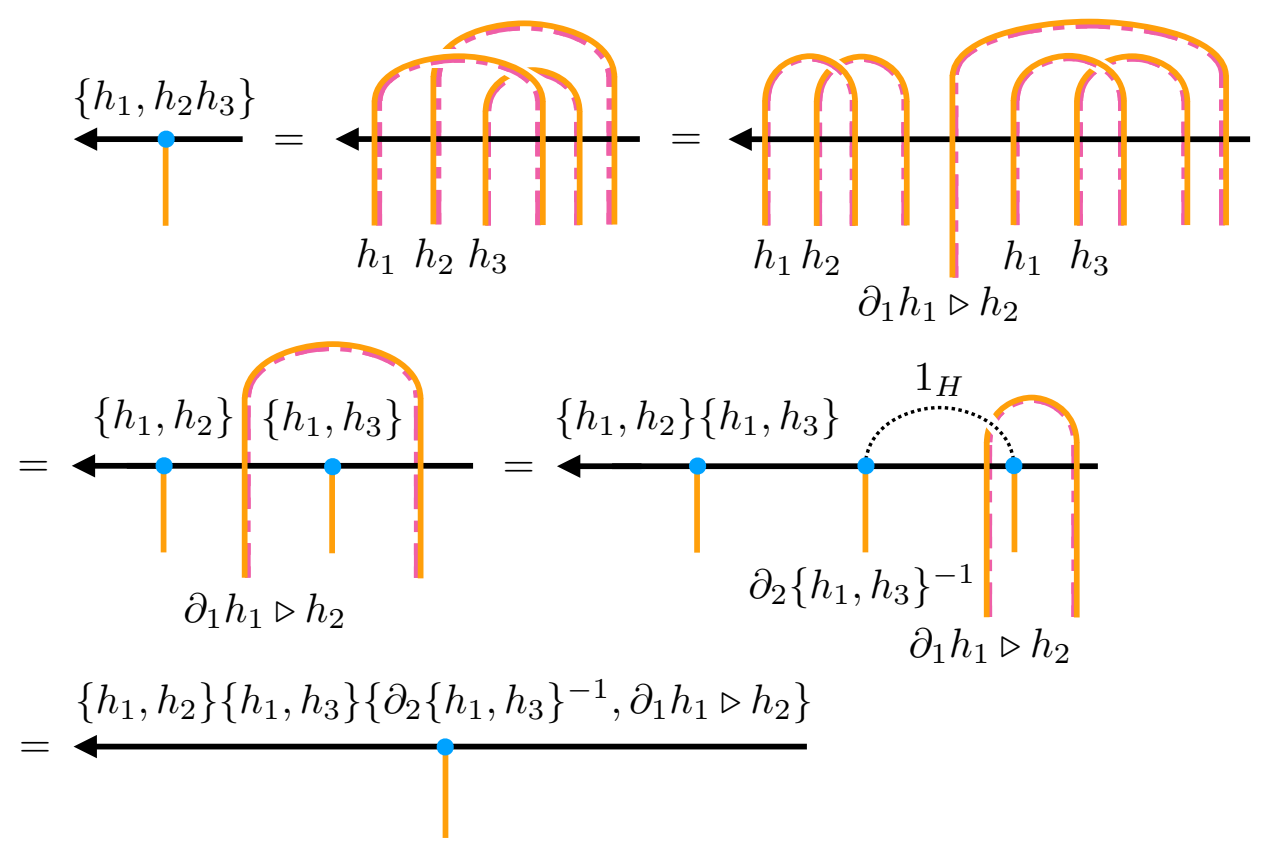

- Equation (C.11):
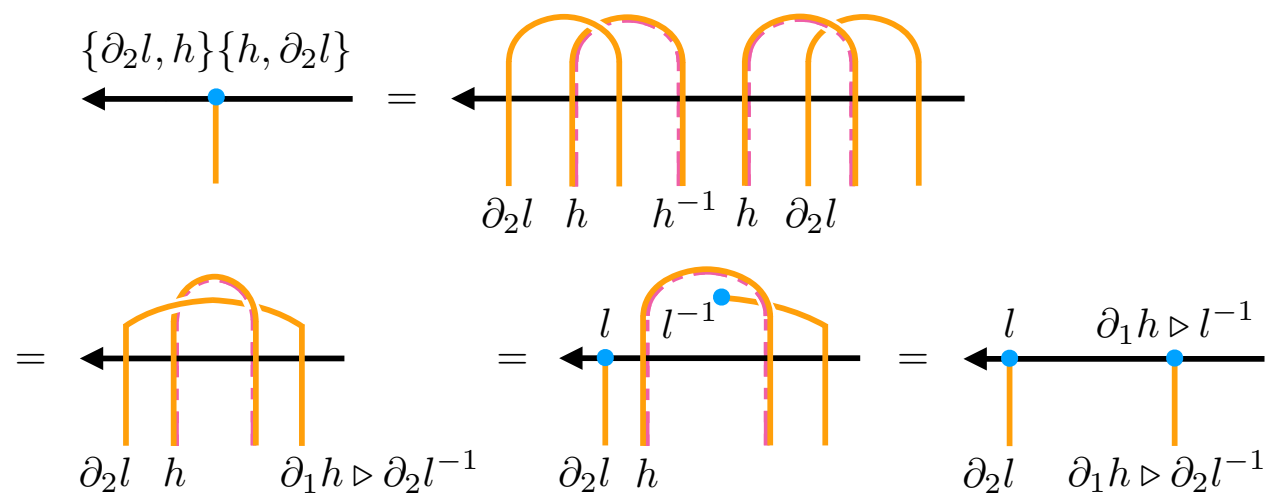

In order to obtain the last equation, we have used eq. (D.16).

\section{D.5 $(L, H)$ as 2-group}

We have shown that the 3-group can be diagrammatically expressed. By using them, we can also describe the fact that the set $\left(L \stackrel{\partial_{2}}{\rightarrow} H, \triangleright^{\prime}\right)$ is a 2-group (see appendix C.1). Here, the action $\triangleright^{\prime}$ of $H$ on $H$ and $L$ are defined by conjugation $h \triangleright^{\prime} h^{\prime}=h h^{\prime} h^{-1}$ and by the Peiffer lifting $h \triangleright^{\prime} l=l\left\{\partial_{2} l^{-1}, h\right\}$, respectively. In order to reproduce the 2-group structure, we should diagrammatically show the action $\triangleright^{\prime}$, the compatibility of $\partial_{2}$ with $\triangleright^{\prime}$, and the Peiffer identity $l_{1} l_{2} l_{1}^{-1}=\left(\partial_{2} l_{1}\right) \triangleright^{\prime} l_{2}$.

One of the advantages of the diagrammatic expression is that we can straightforwardly reproduce them, in particular the action $\triangleright^{\prime}$, which may be complicated. Let us express the 
definition of the action $\triangleright^{\prime}$. As in the definition of the action $\triangleright$ for the 3-group, we can describe the action $h \triangleright^{\prime} h^{\prime}$ and $h \triangleright^{\prime} l$ by enclosing $h \in H$ and $l \in L$ with $h \in H$, respectively. First, we consider the action of $h \in H$ on $h^{\prime} \in H$ defined by conjugation $h \triangleright^{\prime} h^{\prime}=h h^{\prime} h^{-1}$, which can be expressed as follows:

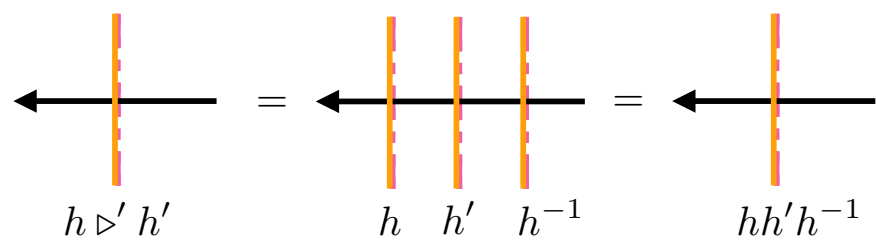

Second, we can simply reproduce the definition $h \triangleright^{\prime} l=l\left\{\partial_{2} l^{-1}, h\right\}$ by using the following the deformations:

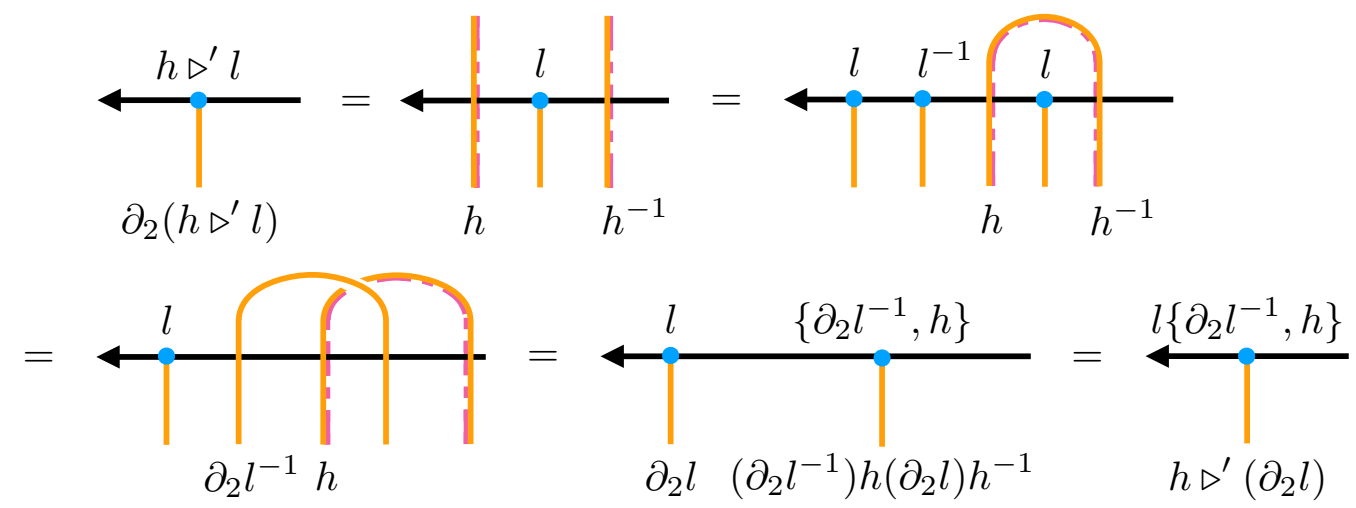

Here, we have used eq. (D.17) in order to have the fourth expression. The above deformations automatically show the compatibility of $\partial_{2}$ with the action of $H: \partial_{2}\left(h \triangleright^{\prime} l\right)=$ $h \triangleright^{\prime}\left(\partial_{2} l\right)=h\left(\partial_{2} l\right) h^{-1}$.

The 2-group should satisfy the Peiffer identity $l_{1} l_{2} l_{1}^{-1}=\partial_{2} l_{1} \triangleright^{\prime} l_{2}$, which can now be shown as follows:

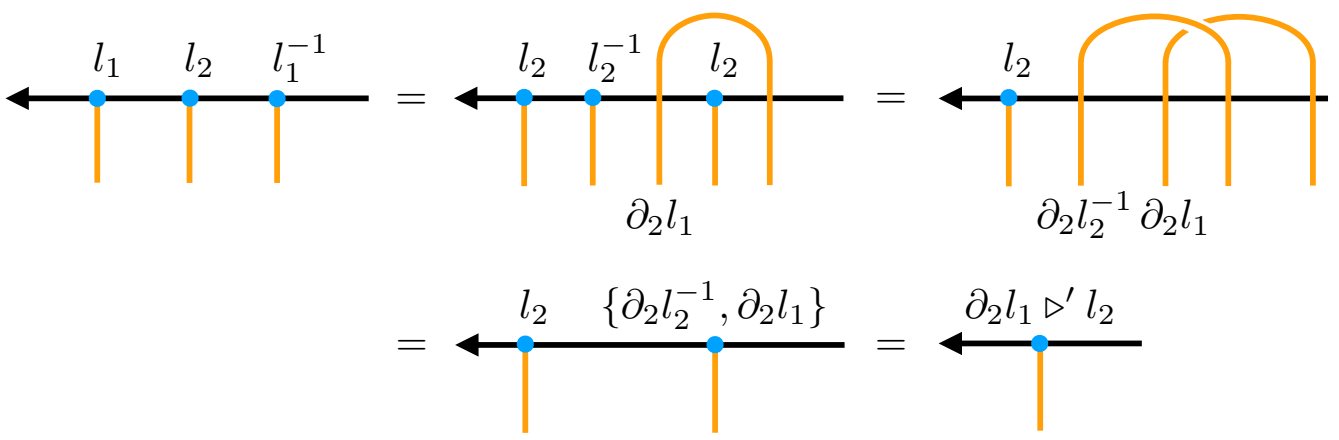

Here, we have used eq. (D.17) in the first line.

\section{D.6 Global 3-group symmetry and symmetry generators}

Finally, we consider how to relate the above diagrammatic expressions to the symmetry generators of the higher-form global symmetries given by the 3-group. In the following, we identify $G, H$, and $L$ as symmetry groups which parameterize the 0-, 1-, and 2-form 
symmetries, respectively. In the following, we discuss symmetry generators for the higherform symmetries which do not have interiors, and are not boundaries of other objects. The assumptions restrict the symmetry group that non-trivially parameterizes the symmetry generators. Further, we assume that the restricted group also has a 3-group structure. As we will show in appendix D.6.5, the assumptions require that the symmetry groups $G, H$, and $L$ are reduced to

$$
G_{\text {gl. }}:=G / \operatorname{Im} \partial_{1}, \quad H_{\text {gl. }}:=H_{\mathrm{Ab} .} / \operatorname{Im} \partial_{2}, \quad L_{\text {gl. }}:=\operatorname{Ker} \partial_{2},
$$

respectively. Here, $H_{\mathrm{Ab}}$. is the Abelian part of Ker $\partial_{1} \subset H$. This assumption is sufficient to consider the symmetry generators of the axion electrodynamics.

\section{D.6.1 Symmetry transformations}

Let us recall the symmetry transformations in the higher-form symmetries. For elements of the groups $g \in G_{\text {gl. }}, h \in H_{\text {gl. }}$ and $l \in L_{\text {gl. }}$, the corresponding symmetry generators are expressed by topological objects $U_{0}(g, \mathcal{S}), U_{1}(h, \mathcal{C})$, and $U_{2}\left(l,\left(\mathcal{P}, \mathcal{P}^{\prime}\right)\right)$, respectively. Here, $\mathcal{S}, \mathcal{C}$, and $\left(\mathcal{P}, \mathcal{P}^{\prime}\right)$ are a closed surface, a closed line and two points. The symmetry generators can act on the 0-, 1-, and 2-dimensional charged objects $\Phi\left(\mathcal{P}_{\Phi}\right), W\left(\mathcal{C}_{W}\right)$, and $V\left(\mathcal{S}_{V}\right)$ as unitary representations:

$$
\begin{aligned}
& \left\langle U_{0}(g, \mathcal{S}) \Phi\left(\mathcal{P}_{\Phi}\right)\right\rangle=R_{0}(g)\left\langle\Phi\left(\mathcal{P}_{\Phi}\right)\right\rangle \quad \text { if } \quad \operatorname{Link}\left(\mathcal{S}, \mathcal{P}_{\Phi}\right)=1, \\
& \left\langle U_{1}(h, \mathcal{C}) W\left(\mathcal{C}_{W}\right)\right\rangle=R_{1}(h)\left\langle W\left(\mathcal{C}_{W}\right)\right\rangle \quad \text { if } \quad \operatorname{Link}\left(\mathcal{C}, \mathcal{C}_{W}\right)=1, \\
& \left\langle U_{2}\left(l,\left(\mathcal{P}, \mathcal{P}^{\prime}\right)\right) V\left(\mathcal{S}_{V}\right)\right\rangle=R_{2}(l)\left\langle V\left(\mathcal{S}_{V}\right)\right\rangle \quad \text { if } \quad \operatorname{Link}\left(\left(\mathcal{P}, \mathcal{P}^{\prime}\right), \mathcal{S}_{V}\right)=1,
\end{aligned}
$$

respectively. Here, $\mathcal{P}_{\Phi}, \mathcal{C}_{W}$, and $\mathcal{S}_{V}$ are a point, a closed line, and a closed surface. We denote $R_{0}(g), R_{1}(h)$, and $R_{2}(l)$ as unitary representation matrices (c-number) of $g, h$, and $l$, respectively.

\section{D.6.2 Diagrammatic expressions of symmetry transformations}

We now show the diagrammatic expressions of the symmetry generators and their symmetry transformations. The charged objects can be diagrammatically expressed as follows.

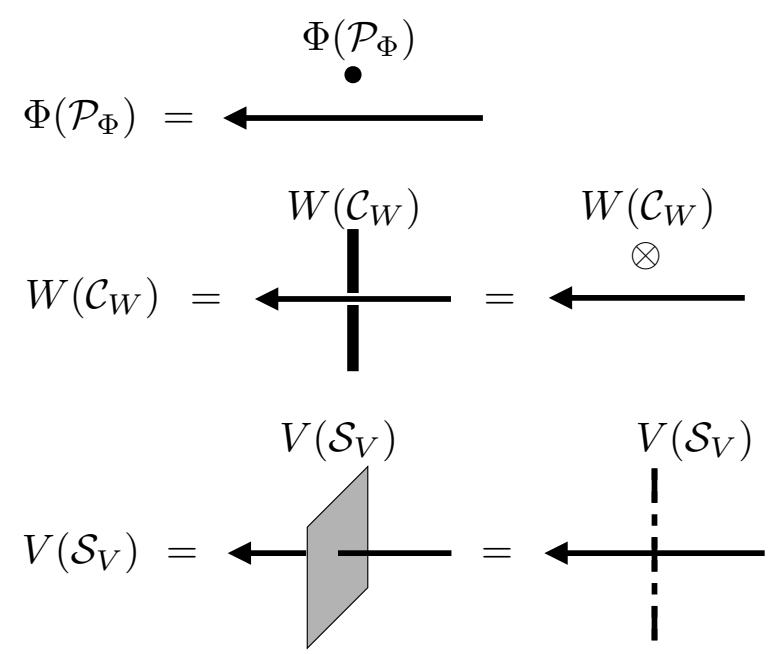

The right-hand sides of eqs. (D.37) and (D.38) are projected diagrams. 
As in the ordinary quantum mechanics, the symmetry generators can be unitary representations of the symmetry groups which preserve the group structures. This implies that we can simply replace the diagrammatic expressions of the $G_{\mathrm{gl}}, H_{\mathrm{gl} .}$, and $L_{\mathrm{gl}}$. with the 0-, 1-, and 2-form symmetry generators. For the 0-form symmetry, the unitary representation in eq. (D.33) can be described as follows:

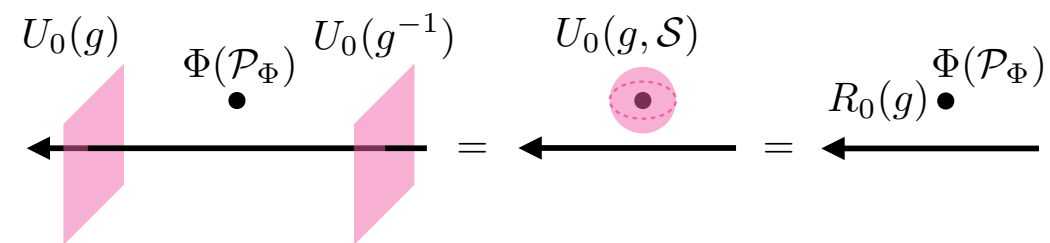

Here, we have enclosed $\Phi\left(\mathcal{P}_{\Phi}\right)$ by surfaces $U_{0}(g)$ and $U_{0}\left(g^{-1}\right)$ parameterized by $g$ and $g^{-1}$, respectively. Since $U_{0}(g)$ are topological, we can deform $U_{0}(g)$ and $U_{0}\left(g^{-1}\right)$ to $U_{0}(g, \mathcal{S})$ given by a surface $\mathcal{S}$. The projections of the diagram can be shown as follows:

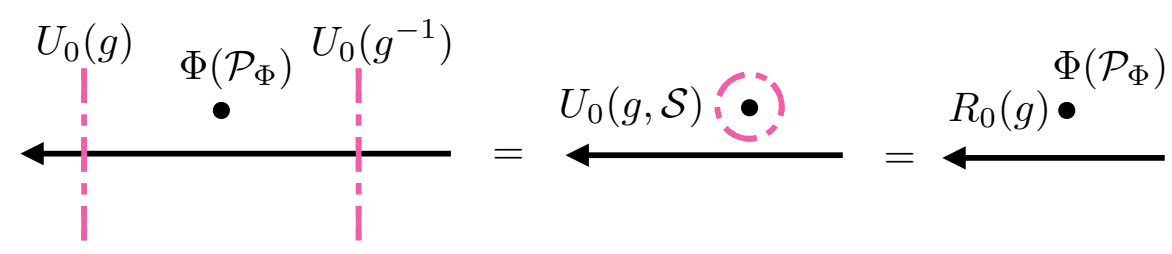

For the 1-form symmetry, the diagram for the unitary representation in eq. (D.34) and the projection of the diagram are respectively given as follows:

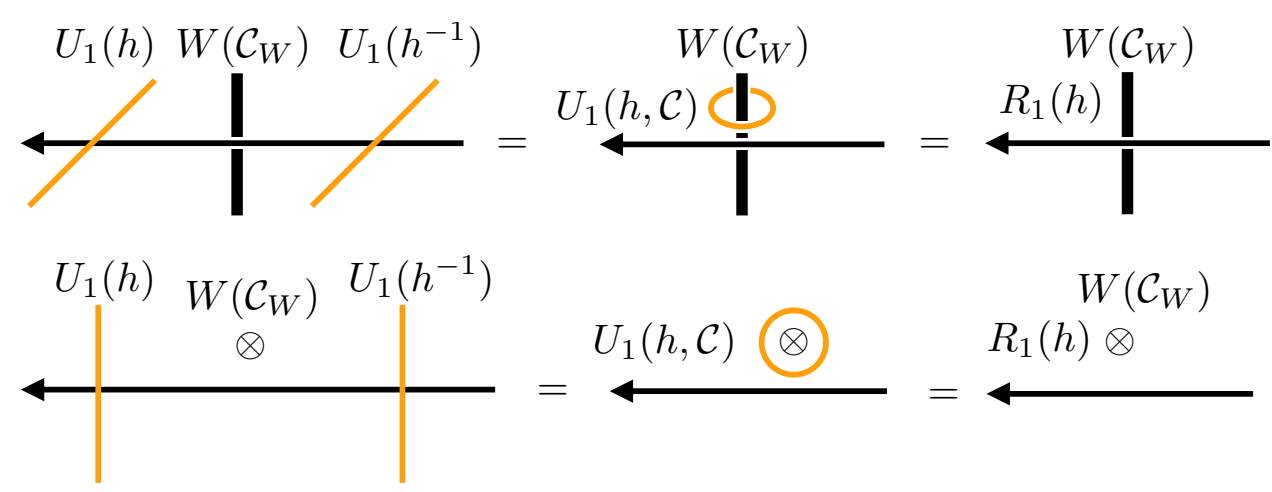

For the 2-form symmetry, the unitary representation in eq. (D.35) can be visualized by the following diagram and its projection:

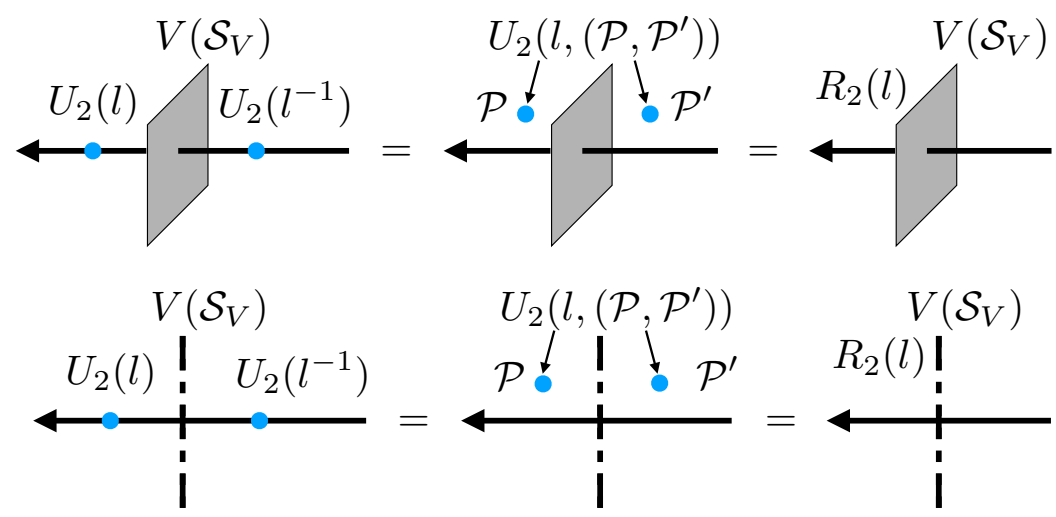

Here, the $\mathcal{P}^{\prime}$ has an orientation opposite to the point $\mathcal{P}$. 


\section{D.6.3 Diagrammatic expression of actions}

Now we consider the diagrammatic expression of the symmetry generators parameterized by the action $\triangleright$. For the 0 -form symmetry, the symmetry generator $U_{0}\left(g \triangleright g^{\prime}, \mathcal{S}\right)$ is equal to $U_{0}\left(g g^{\prime} g^{-1}, \mathcal{S}\right)$, and the diagram is reduced to eq. (D.39). The symmetry generators $U_{1}(g \triangleright h, \mathcal{C})$ and $U_{2}\left(g \triangleright l,\left(\mathcal{P}, \mathcal{P}^{\prime}\right)\right)$ can be respectively expressed as follows:

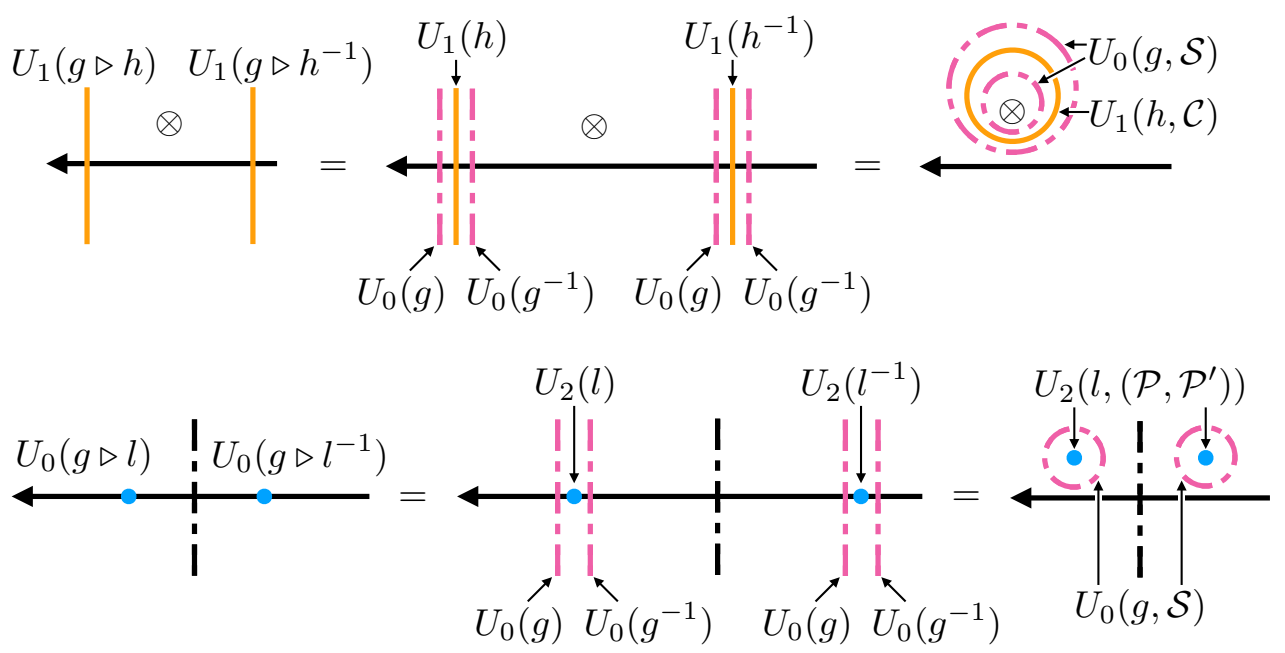

In the diagram in eq. (D.45), $\mathcal{S}$ is a set of two cylinders, and the orientation of the cylinder inside $U_{1}(h, \mathcal{C})$ is opposite to the one outside $U_{1}(h, \mathcal{C})$. Note that this diagram can express the Witten effect for the axionic domain walls [101], which has been discussed in eq. (4.1).

\section{D.6.4 Diagrammatic expression of Peiffer lifting}

Here, we show the diagrammatic expression of the Peiffer lifting for the symmetry generators. We can describe the symmetry generator $U_{2}\left(\left\{h, h^{\prime}\right\},\left(\mathcal{P}, \mathcal{P}^{\prime}\right)\right)$ for the elements $h, h^{\prime} \in H_{\text {gl. }}=H_{\mathrm{Ab}} / \operatorname{Im} \partial_{1}$ by using eqs. (D.43) or (D.44).

As we discussed in appendix D.4, the Peiffer lifting is related to the braiding of the elements $h, h^{\prime} \in H_{\mathrm{gl}}$. In the case of the symmetry generators, the Peiffer lifting is related to the linking of two 1-form symmetry generators. Before discussing the symmetry generators, we consider the linking of group elements. For the elements $h, h^{\prime} \in H_{\text {gl. }}$, we can construct linking of $h$ and $h^{\prime}$ from the Peiffer lifting $\left\{h, h^{\prime}\right\}\left\{h^{\prime}, h\right\}$ :
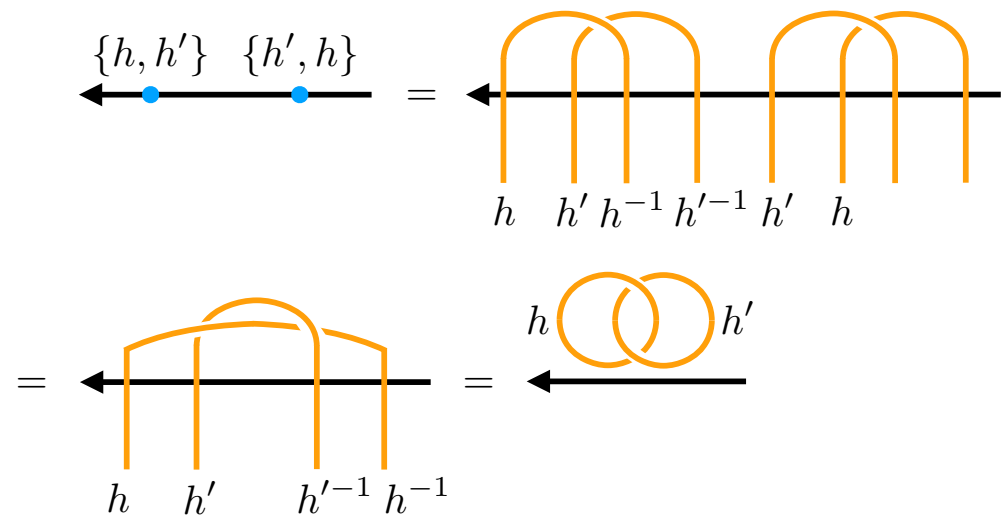
Now, we discuss the linking of the symmetry generators. By the diagram in eq. (D.47), we find that the linking of the 1-form symmetry generators leads to 2 -form symmetry generators, which can act on $V\left(\mathcal{S}_{V}\right)$ :

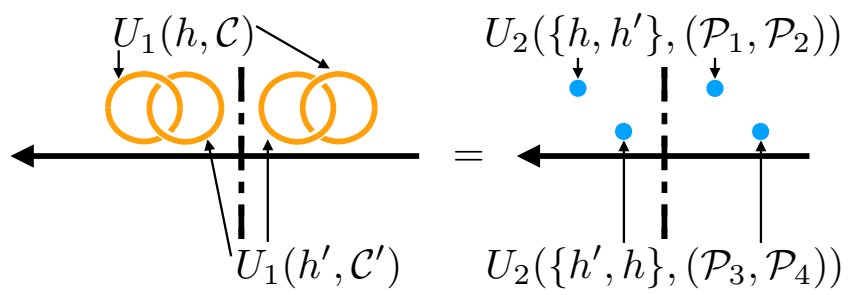

Note that the diagrammatic expression can describe the anomalous effect around the axionic strings [101], which has been discussed in eq. (4.3).

\section{D.6.5 Symmetry groups parameterizing symmetry generators}

Here, we show that the groups which parameterize the symmetry generators are not the groups $G, H$, and $L$ themselves but subgroups of them. The subgroups are specified as $G / \operatorname{Im} \partial_{1}, H_{\mathrm{Ab}} / / \operatorname{Im} \partial_{2}$ and $\operatorname{Ker} \partial_{2}$, where $H_{\mathrm{Ab}}$. is an Abelian part of Ker $\partial_{1} \subset H$.

We require three assumptions for the symmetry generators. One is that the non-trivial symmetry generators are not boundaries of other objects. Another is that the non-trivial symmetry generators do not have boundaries. The last one is that the restricted groups also have the 3 -group structure.

Let us specify the subgroups by using the assumptions. By the first assumption, the symmetry generators are parameterized by the elements of $L, H$, and $G$ satisfying $\partial_{2} l=1_{H}$ and $\partial_{1} h=1_{G}$. Therefore, the symmetry groups are reduced to $G$, Ker $\partial_{1} \subset H$, Ker $\partial_{2} \subset L$. Note that we can consistently define the actions of $G$ on $G$, Ker $\partial_{1}$ and Ker $\partial_{2}$, since the elements $h \in \operatorname{Ker} \partial_{1}$ and $l \in \operatorname{Ker} \partial_{2}$ satisfy $\partial_{1}(g \triangleright h)=g \triangleright \partial_{1} h=1_{G}$ and $\partial_{2}(g \triangleright l)=g \triangleright \partial_{2} l=1_{H}$, and $\partial_{2}\left(h \triangleright^{\prime} l\right)=h \triangleright^{\prime} \partial_{2} l=1_{H}$ for $g \in G$.

The axiom of the Peiffer lifting gives us some properties of the subsets. In particular, the subset Ker $\partial_{2}$ must be Abelian by the axiom in eq. (C.8). The last assumption requires that the Peiffer lifting $\left\{h, h^{\prime}\right\}$ for the elements $h, h^{\prime} \in \operatorname{Ker} \partial_{1}$ should belong to Ker $\partial_{2}$, i.e., $\partial_{2}\left\{h, h^{\prime}\right\}=1_{H}$. By the axiom in eq. (C.6), we have $h h^{\prime}=h^{\prime} h$. Therefore, the Abelian part of Ker $\partial_{1}$ contributes to the higher-form symmetries. In the following, we denote the Abelian part of Ker $\partial_{1}$ as $H_{\mathrm{Ab}}$. Note that these restrictions are consistent with the fact that the $p$-form symmetries $(p>0)$ must be Abelian (except for theories on manifolds with non-trivial topology) [42].

The second assumption implies that the images of $\partial_{1}$ and $\partial_{2}$ cannot parameterize nontrivial symmetry generators. The reason can be simply understood by using our diagrams: the symmetry generators given by $\partial_{1} h$ and $\partial_{2} l$ can be annihilated by pair creations of $h$ and $l$. For example, the annihilation of $U_{1}\left(\partial_{2} l, \mathcal{C}\right)$ can be seen as follows:

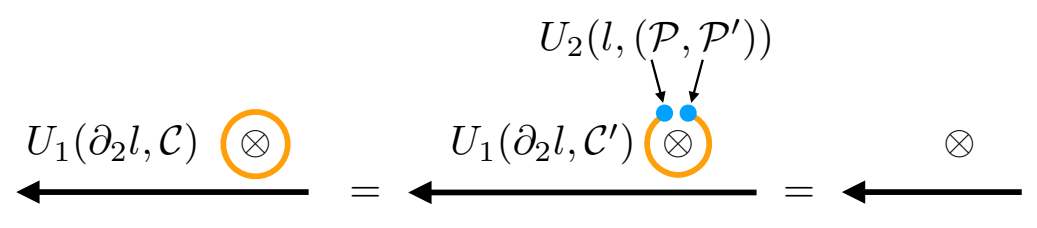


Here, $\mathcal{C}^{\prime}$ is a line whose boundaries are $\mathcal{P}$ and $\mathcal{P}^{\prime}$. The groups parameterizing the symmetry generators are therefore $G / \operatorname{Im} \partial_{1}$ and $H_{\mathrm{Ab}} / / \operatorname{Im} \partial_{2}$, Ker $\partial_{2}$. Note that $G / \operatorname{Im} \partial_{1}$ and $H_{\mathrm{Ab}} / \operatorname{Im} \partial_{2}$ are groups, since $\operatorname{Im} \partial_{1}$ and $\operatorname{Im} \partial_{2}$ are normal subgroups of $G$ and $H$, $g\left(\partial_{1} h\right) g^{-1}=\partial_{1}(g \triangleright h) \in \operatorname{Im} \partial_{1}$ and $h\left(\partial_{2} l\right) h^{-1}=\partial_{2}\left(h \triangleright^{\prime} l\right) \in \operatorname{Im} \partial_{2}$ for $g \in G$ and $h \in H$, respectively.

In summary, the 0-, 1-, and 2-form symmetry generators are parameterized by $G / \operatorname{Im} \partial_{1}, H_{\mathrm{Ab}} / \operatorname{Im} \partial_{2}$ and $\operatorname{Ker} \partial_{2}$.

Open Access. This article is distributed under the terms of the Creative Commons Attribution License (CC-BY 4.0), which permits any use, distribution and reproduction in any medium, provided the original author(s) and source are credited.

\section{References}

[1] R.D. Peccei and H.R. Quinn, CP conservation in the presence of instantons, Phys. Rev. Lett. 38 (1977) 1440 [INSPIRE].

[2] S. Weinberg, A new light boson?, Phys. Rev. Lett. 40 (1978) 223 [INSPIRE].

[3] F. Wilczek, Problem of strong $P$ and $T$ invariance in the presence of instantons, Phys. Rev. Lett. 40 (1978) 279 [inSPIRE].

[4] M. Dine, W. Fischler and M. Srednicki, A simple solution to the strong CP problem with a harmless axion, Phys. Lett. B 104 (1981) 199 [INSPIRE].

[5] A.R. Zhitnitsky, On possible suppression of the axion hadron interactions (in Russian), Sov. J. Nucl. Phys. 31 (1980) 260 [Yad. Fiz. 31 (1980) 497] [inSPIRE].

[6] J.E. Kim, Weak interaction singlet and strong CP invariance, Phys. Rev. Lett. 43 (1979) 103 [INSPIRE].

[7] M.A. Shifman, A.I. Vainshtein and V.I. Zakharov, Can confinement ensure natural CP invariance of strong interactions?, Nucl. Phys. B 166 (1980) 493 [INSPIRE].

[8] J.E. Kim, Light pseudoscalars, particle physics and cosmology, Phys. Rept. 150 (1987) 1 [INSPIRE].

[9] M. Dine, TASI lectures on the strong CP problem, hep-ph/0011376 [INSPIRE].

[10] R.D. Peccei, The strong CP problem and axions, Lect. Notes Phys. 741 (2008) 3 [hep-ph/0607268] [INSPIRE].

[11] M. Kawasaki and K. Nakayama, Axions: theory and cosmological role, Ann. Rev. Nucl. Part. Sci. 63 (2013) 69 [arXiv:1301.1123] [inSPIRE].

[12] F. Wilczek, Two applications of axion electrodynamics, Phys. Rev. Lett. 58 (1987) 1799 [INSPIRE].

[13] X.-L. Qi, T. Hughes and S.-C. Zhang, Topological field theory of time-reversal invariant insulators, Phys. Rev. B 78 (2008) 195424 [arXiv:0802.3537] [INSPIRE].

[14] A.M. Essin, J.E. Moore and D. Vanderbilt, Magnetoelectric polarizability and axion electrodynamics in crystalline insulators, Phys. Rev. Lett. 102 (2009) 146805 [arXiv:0810.2998] [INSPIRE]. 
[15] M.Z. Hasan and C.L. Kane, Topological insulators, Rev. Mod. Phys. 82 (2010) 3045 [arXiv: 1002.3895] [INSPIRE].

[16] W. Fischler and J. Preskill, Dyon-axion dynamics, Phys. Lett. B 125 (1983) 165 [InSPIRE].

[17] P. Sikivie, On the interaction of magnetic monopoles with axionic domain walls, Phys. Lett. B 137 (1984) 353 [INSPIRE].

[18] P. Sikivie, Of axions, domain walls and the early universe, Phys. Rev. Lett. 48 (1982) 1156 [INSPIRE].

[19] A. Vilenkin and A.E. Everett, Cosmic strings and domain walls in models with Goldstone and pseudo-Goldstone bosons, Phys. Rev. Lett. 48 (1982) 1867 [InSPIRE].

[20] R.L. Davis, Cosmic axions from cosmic strings, Phys. Lett. B 180 (1986) 225 [INSPIRE].

[21] J.C.Y. Teo and C.L. Kane, Topological defects and gapless modes in insulators and superconductors, Phys. Rev. B 82 (2010) 115120 [arXiv: 1006.0690] [INSPIRE].

[22] E. Witten, Dyons of charge e $\theta / 2 \pi$, Phys. Lett. B 86 (1979) 283 [InSPIRE].

[23] I.I. Kogan, Kaluza-Klein and axion domain walls: Induced charge and mass transmutation, Phys. Lett. B 299 (1993) 16 [InSPIRE].

[24] I.I. Kogan, Axions, monopoles and cosmic strings, hep-ph/9305307 [INSPIRE].

[25] E.J. Ferrer and V. de la Incera, Novel topological effects in dense QCD in a magnetic field, Nucl. Phys. B 931 (2018) 192 [arXiv:1512.03972] [INSPIRE].

[26] N. Yamamoto, Axion electrodynamics and nonrelativistic photons in nuclear and quark matter, Phys. Rev. D 93 (2016) 085036 [arXiv:1512.05668] [INSPIRE].

[27] E.J. Ferrer and V. de la Incera, Dissipationless Hall current in dense quark matter in a magnetic field, Phys. Lett. B $\mathbf{7 6 9}$ (2017) 208 [arXiv:1611.00660] [InSPIRE].

[28] Z. Qiu, G. Cao and X.-G. Huang, On electrodynamics of chiral matter, Phys. Rev. D 95 (2017) 036002 [arXiv: 1612.06364] [INSPIRE].

[29] G. Bednik, A.A. Zyuzin and A.A. Burkov, Anomalous Hall effect in Weyl superconductors, New J. Phys. 18 (2016) 085002 [arXiv: 1605.05734] [InSPIRE].

[30] Z. Wang and S.-C. Zhang, Chiral anomaly, charge density waves, and axion strings from Weyl semimetals, Phys. Rev. B 87 (2013) 161107 [arXiv:1207.5234] [INSPIRE].

[31] C.G. Callan, Jr. and J.A. Harvey, Anomalies and Fermion zero modes on strings and domain walls, Nucl. Phys. B 250 (1985) 427 [INSPIRE].

[32] S.G. Naculich, Axionic strings: covariant anomalies and bosonization of chiral zero modes, Nucl. Phys. B 296 (1988) 837 [InSPIRE].

[33] L. Álvarez-Gaumé and P.H. Ginsparg, The topological meaning of non-Abelian anomalies, Nucl. Phys. B 243 (1984) 449 [InSPIRE].

[34] D.B. Kaplan and A. Manohar, Anomalous vortices and electromagnetism, Nucl. Phys. B 302 (1988) 280 [inSPIRE].

[35] A. Manohar, Anomalous vortices and electromagnetism. II., Phys. Lett. B 206 (1988) 276 [Erratum ibid. 209 (1988) 543] [INSPIRE].

[36] J.A. Harvey and S.G. Naculich, Cosmic strings from pseudoanomalous U(1)s, Phys. Lett. B 217 (1989) 231 [INSPIRE]. 
[37] P.K. Townsend, Effective description of axion defects, Phys. Lett. B 309 (1993) 33 [hep-th/9303171] [INSPIRE].

[38] J.M. Izquierdo and P.K. Townsend, Axionic defect anomalies and their cancellation, Nucl. Phys. B 414 (1994) 93 [hep-th/9307050] [INSPIRE].

[39] J.A. Harvey and O. Ruchayskiy, The local structure of anomaly inflow, JHEP 06 (2001) 044 [hep-th/0007037] [INSPIRE].

[40] T. Banks and N. Seiberg, Symmetries and strings in field theory and gravity, Phys. Rev. D 83 (2011) 084019 [arXiv:1011.5120] [INSPIRE].

[41] A. Kapustin and N. Seiberg, Coupling a QFT to a TQFT and duality, JHEP 04 (2014) 001 [arXiv: 1401.0740] [INSPIRE].

[42] D. Gaiotto, A. Kapustin, N. Seiberg and B. Willett, Generalized global symmetries, JHEP 02 (2015) 172 [arXiv: 1412.5148] [INSPIRE].

[43] C.D. Batista and Z. Nussinov, Generalized Elitzur's theorem and dimensional reduction, Phys. Rev. B 72 (2005) 045137 [cond-mat/0410599] [INSPIRE].

[44] T. Pantev and E. Sharpe, GLSM's for Gerbes (and other toric stacks), Adv. Theor. Math. Phys. 10 (2006) 77 [hep-th/0502053] [inSPIRE].

[45] T. Pantev and E. Sharpe, String compactifications on Calabi-Yau stacks, Nucl. Phys. B 733 (2006) 233 [hep-th/0502044] [INSPIRE].

[46] T. Pantev and E. Sharpe, Notes on gauging noneffective group actions, hep-th/0502027 [INSPIRE].

[47] Z. Nussinov and G. Ortiz, Sufficient symmetry conditions for Topological Quantum Order, Proc. Nat. Acad. Sci. 106 (2009) 16944 [cond-mat/0605316] [INSPIRE].

[48] Z. Nussinov and G. Ortiz, Autocorrelations and thermal fragility of anyonic loops in topologically quantum ordered systems, Phys. Rev. B 77 (2008) 064302 [arXiv:0709.2717].

[49] Z. Nussinov and G. Ortiz, A symmetry principle for topological quantum order, Annals Phys. 324 (2009) 977 [cond-mat/0702377] [INSPIRE].

[50] Z. Nussinov, G. Ortiz and E. Cobanera, Effective and exact holographies from symmetries and dualities, Annals Phys. 327 (2012) 2491 [arXiv:1110.2179] [INSPIRE].

[51] J. Distler and E. Sharpe, Quantization of Fayet-Iliopoulos parameters in supergravity, Phys. Rev. D 83 (2011) 085010 [arXiv: 1008.0419] [INSPIRE].

[52] A. Kovner and B. Rosenstein, New look at QED in four-dimensions: The Photon as a Goldstone boson and the topological interpretation of electric charge, Phys. Rev. D 49 (1994) 5571 [hep-th/9210154] [INSPIRE].

[53] E. Lake, Higher-form symmetries and spontaneous symmetry breaking, arXiv:1802.07747 [INSPIRE].

[54] S. Ozaki and N. Yamamoto, Axion crystals, JHEP 08 (2017) 098 [arXiv:1610.07835] [INSPIRE].

[55] N. Sogabe and N. Yamamoto, Triangle anomalies and nonrelativistic Nambu-Goldstone modes of generalized global symmetries, Phys. Rev. D 99 (2019) 125003

[arXiv: 1903. 02846] [INSPIRE]. 
[56] Y. Hidaka, Y. Hirono and R. Yokokura, Counting Nambu-Goldstone modes of higher-form global symmetries, arXiv:2007.15901 [INSPIRE].

[57] X.G. Wen, Topological order in rigid states, Int. J. Mod. Phys. B 4 (1990) 239 [inSPIRE].

[58] X.G. Wen and Q. Niu, Ground-state degeneracy of the fractional quantum Hall states in the presence of a random potential and on high-genus Riemann surfaces, Phys. Rev. B $4 \mathbf{1}$ (1990) 9377 [INSPIRE].

[59] X.-G. Wen, Topological orders and Chern-Simons theory in strongly correlated quantum liquid, Int. J. Mod. Phys. B 5 (1991) 1641 [INSPIRE].

[60] T.H. Hansson, V. Oganesyan and S.L. Sondhi, Superconductors are topologically ordered, Annals Phys. 313 (2004) 497 [cond-mat/0404327] [INSPIRE].

[61] D. Gaiotto, A. Kapustin, Z. Komargodski and N. Seiberg, Theta, time reversal, and temperature, JHEP 05 (2017) 091 [arXiv: 1703.00501] [INSPIRE].

[62] D. Gaiotto, Z. Komargodski and N. Seiberg, Time-reversal breaking in $Q C D_{4}$, walls, and dualities in $2+1$ dimensions, JHEP 01 (2018) 110 [arXiv:1708.06806] [INSPIRE].

[63] Y. Tanizaki, T. Misumi and N. Sakai, Circle compactification and 't Hooft anomaly, JHEP 12 (2017) 056 [arXiv: 1710.08923] [INSPIRE].

[64] Y. Tanizaki, Y. Kikuchi, T. Misumi and N. Sakai, Anomaly matching for the phase diagram of massless $\mathbb{Z}_{N}-Q C D$, Phys. Rev. D 97 (2018) 054012 [arXiv:1711.10487] [InSPIRE].

[65] Z. Komargodski, A. Sharon, R. Thorngren and X. Zhou, Comments on Abelian Higgs models and persistent order, SciPost Phys. 6 (2019) 003 [arXiv:1705. 04786] [INSPIRE].

[66] Y. Hirono and Y. Tanizaki, Quark-hadron continuity beyond the Ginzburg-Landau paradigm, Phys. Rev. Lett. 122 (2019) 212001 [arXiv:1811.10608] [INSPIRE].

[67] Y. Hirono and Y. Tanizaki, Effective gauge theories of superfluidity with topological order, JHEP 07 (2019) 062 [arXiv:1904.08570] [INSPIRE].

[68] Y. Hidaka, Y. Hirono, M. Nitta, Y. Tanizaki and R. Yokokura, Topological order in the color-flavor locked phase of a $(3+1)$-dimensional $\mathrm{U}(N)$ gauge-Higgs system, Phys. Rev. D 100 (2019) 125016 [arXiv: 1903. 06389] [INSPIRE].

[69] M.M. Anber and E. Poppitz, On the baryon-color-flavor (BCF) anomaly in vector-like theories, JHEP 11 (2019) 063 [arXiv: 1909.09027] [INSPIRE].

[70] T. Misumi, Y. Tanizaki and M. Ünsal, Fractional $\theta$ angle, 't Hooft anomaly, and quantum instantons in charge-q multi-flavor Schwinger model, JHEP 07 (2019) 018 [arXiv: 1905. 05781] [INSPIRE].

[71] M.M. Anber and E. Poppitz, Deconfinement on axion domain walls, JHEP 03 (2020) 124 [arXiv: 2001.03631] [INSPIRE].

[72] M.M. Anber and E. Poppitz, Generalized 't Hooft anomalies on non-spin manifolds, JHEP 04 (2020) 097 [arXiv: 2002. 02037] [INSPIRE].

[73] T. Furusawa and M. Hongo, Global anomaly matching in the higher-dimensional $\mathbb{C P}^{N-1}$ model, Phys. Rev. B 101 (2020) 155113 [arXiv:2001.07373] [inSPIRE].

[74] E. Sharpe, Notes on generalized global symmetries in QFT, Fortsch. Phys. 63 (2015) 659 [arXiv: 1508.04770] [INSPIRE].

[75] J.C. Baez, Higher Yang-Mills theory, hep-th/0206130 [INSPIRE]. 
[76] J.C. Baez and A.S. Crans, Higher-dimensional algebra VI: Lie 2-algebras, Theor. Appl. Categor. 12 (2004) 492 [math/0307263] [INSPIRE].

[77] J. Baez and U. Schreiber, Higher gauge theory: 2-connections on 2-bundles, hep-th/0412325 [INSPIRE].

[78] J.C. Baez and U. Schreiber, Higher gauge theory, math/0511710 [INSPIRE].

[79] J.F. Martins and R. Picken, The fundamental Gray 3-groupoid of a smooth manifold and local 3-dimensional holonomy based on a 2-crossed module, arXiv:0907.2566 [INSPIRE].

[80] J.C. Baez and J. Huerta, An invitation to higher gauge theory, Gen. Rel. Grav. 43 (2011) 2335 [arXiv: 1003.4485] [INSPIRE].

[81] P.-M. Ho and Y. Matsuo, Note on non-Abelian two-form gauge fields, JHEP 09 (2012) 075 [arXiv:1206.5643] [INSPIRE].

[82] C. Säemann and M. Wolf, Six-dimensional superconformal field theories from principal 3-bundles over twistor space, Lett. Math. Phys. 104 (2014) 1147 [arXiv:1305.4870] [INSPIRE].

[83] W. Wang, On 3-gauge transformations, 3-curvatures, and Gray-categories, J. Math. Phys. 55 (2014) 043506 [arXiv:1311.3796] [inSPIRE].

[84] E. Soncini and R. Zucchini, A new formulation of higher parallel transport in higher gauge theory, J. Geom. Phys. 95 (2015) 28 [arXiv:1410.0775] [INSPIRE].

[85] B. Jurčo, C. Sämann and M. Wolf, Semistrict higher gauge theory, JHEP 04 (2015) 087 [arXiv: 1403.7185] [INSPIRE].

[86] B. Jurčo, C. Sämann and M. Wolf, Higher groupoid bundles, higher spaces, and self-dual tensor field equations, Fortsch. Phys. 64 (2016) 674 [arXiv:1604.01639] [INSPIRE].

[87] C. Sämann, Higher structures, self-dual strings and $6 D$ superconformal field theories, Fortsch. Phys. 67 (2019) 1910014 [INSPIRE].

[88] T. Radenkovic and M. Vojinovic, Higher gauge theories based on 3-groups, JHEP 10 (2019) 222 [arXiv: 1904.07566] [INSPIRE].

[89] A. Kapustin and R. Thorngren, Higher symmetry and gapped phases of gauge theories, arXiv: 1309.4721 [INSPIRE].

[90] A. Kapustin and R. Thorngren, Topological field theory on a lattice, discrete theta-angles and confinement, Adv. Theor. Math. Phys. 18 (2014) 1233 [arXiv:1308.2926] [INSPIRE].

[91] L. Bhardwaj, D. Gaiotto and A. Kapustin, State sum constructions of spin-TFTs and string net constructions of fermionic phases of matter, JHEP 04 (2017) 096 [arXiv:1605.01640] [INSPIRE].

[92] R.C. de Almeida, J.P. Ibieta-Jimenez, J.L. Espiro and P. Teotonio-Sobrinho, Topological Order from a Cohomological and Higher Gauge Theory perspective, arXiv:1711.04186 [INSPIRE].

[93] C. Delcamp and A. Tiwari, From gauge to higher gauge models of topological phases, JHEP 10 (2018) 049 [arXiv: 1802.10104] [INSPIRE].

[94] C. Delcamp and A. Tiwari, On 2-form gauge models of topological phases, JHEP 05 (2019) 064 [arXiv: 1901.02249] [INSPIRE]. 
[95] R. Thorngren, Topological quantum field theory, symmetry breaking, and finite gauge theory in 3+1D, Phys. Rev. B 101 (2020) 245160 [arXiv:2001.11938] [INSPIRE].

[96] X.-G. Wen, Emergent anomalous higher symmetries from topological order and from dynamical electromagnetic field in condensed matter systems, Phys. Rev. B 99 (2019) 205139 [arXiv: 1812.02517] [INSPIRE].

[97] P.S. Hsin and A. Turzillo, Symmetry-enriched quantum spin liquids in $(3+1) d$, JHEP 09 (2020) 022 [arXiv: 1904.11550].

[98] P.S. Hsin and H.T. Lam, Discrete theta angles, symmetries and anomalies, arXiv:2007.05915.

[99] C. Córdova, T.T. Dumitrescu and K. Intriligator, Exploring 2-group global symmetries, JHEP 02 (2019) 184 [arXiv:1802.04790] [INSPIRE].

[100] Y. Tanizaki and M. Ünsal, Modified instanton sum in QCD and higher-groups, JHEP 03 (2020) 123 [arXiv: 1912.01033] [INSPIRE].

[101] Y. Hidaka, M. Nitta and R. Yokokura, Higher-form symmetries and 3-group in axion electrodynamics, Phys. Lett. B 808 (2020) 135672 [arXiv:2006.12532] [INSPIRE].

[102] D. Conduché, Modules croisés généralisés de longueur 2, J. Pure Appl. Alg. 34 (1984) 155.

[103] G. 't Hooft, Naturalness, chiral symmetry, and spontaneous chiral symmetry breaking, NATO Sci. Ser. B 59 (1980) 135 [INSPIRE].

[104] Y. Frishman, A. Schwimmer, T. Banks and S. Yankielowicz, The axial anomaly and the bound state spectrum in confining theories, Nucl. Phys. B 177 (1981) 157 [InSPIRE].

[105] S.R. Coleman and B. Grossman, 't Hooft's consistency condition as a consequence of analyticity and unitarity, Nucl. Phys. B 203 (1982) 205 [INSPIRE].

[106] Y. Tanizaki, Anomaly constraint on massless QCD and the role of Skyrmions in chiral symmetry breaking, JHEP 08 (2018) 171 [arXiv:1807.07666] [INSPIRE].

[107] F. Benini, C. Córdova and P.-S. Hsin, On 2-group global symmetries and their anomalies, JHEP 03 (2019) 118 [arXiv:1803.09336] [INSPIRE].

[108] C. Córdova, D.S. Freed, H.T. Lam and N. Seiberg, Anomalies in the space of coupling constants and their dynamical applications I, SciPost Phys. 8 (2020) 001

[arXiv: 1905. 09315] [INSPIRE].

[109] C. Córdova, D.S. Freed, H.T. Lam and N. Seiberg, Anomalies in the space of coupling constants and their dynamical applications II, SciPost Phys. 8 (2020) 002 [arXiv: 1905.13361] [INSPIRE].

[110] R. Dijkgraaf and E. Witten, Topological gauge theories and group cohomology, Commun. Math. Phys. 129 (1990) 393 [INSPIRE].

[111] E. Witten, $\mathrm{SL}(2, \mathbb{Z})$ action on three-dimensional conformal field theories with Abelian symmetry, hep-th/0307041 [INSPIRE].

[112] M. Henneaux and C. Teitelboim, Quantization of topological mass in the presence of a magnetic pole, Phys. Rev. Lett. 56 (1986) 689 [INSPIRE].

[113] E. Witten, Fermion path integrals and topological phases, Rev. Mod. Phys. 88 (2016) 035001 [arXiv: 1508.04715] [INSPIRE]. 
[114] K. Yonekura, On the cobordism classification of symmetry protected topological phases, Commun. Math. Phys. 368 (2019) 1121 [arXiv: 1803.10796] [INSPIRE].

[115] E. Witten and K. Yonekura, Anomaly inflow and the $\eta$-invariant, arXiv:1909.08775 [INSPIRE].

[116] T. Brauner and S. Kadam, Anomalous electrodynamics of neutral pion matter in strong magnetic fields, JHEP 03 (2017) 015 [arXiv:1701.06793] [INSPIRE].

[117] N. Yamamoto and R. Yokokura, Topological mass generation in gapless systems, arXiv:2009.07621 [INSPIRE].

[118] Y. Hidaka, M. Nitta and R. Yokokura, Emergent discrete 3-form symmetry and domain walls, Phys. Lett. B 803 (2020) 135290 [arXiv: 1912.02782] [INSPIRE].

[119] H. Kleinert, Multivalued fields. In condensed matter, electromagnetism, and gravitation, World Scientific, Singapore (2008).

[120] E. Witten, On S duality in Abelian gauge theory, Selecta Math. 1 (1995) 383 [hep-th/9505186] [INSPIRE].

[121] K.-M. Lee, The dual formulation of cosmic strings and vortices, Phys. Rev. D 48 (1993) 2493 [hep-th/9301102] [INSPIRE].

[122] J.S. Carter, S. Kamada, M. Saito and S. Satoh, A theorem of sanderson on link bordisms in dimension 4, Algebr. Geom. Topol. 1 (2001) 299.

[123] I. Nakamura, Surface links with free abelian groups, J. Math. Soc. Jpn. 66 (2014) 247 [arXiv: 0911.4235].

[124] S. Palmer, Higher gauge theory and M-theory, Ph.D. thesis, Heriot-Watt University, Edinburgh, U.K. (2014), arXiv: 1407.0298 [INSPIRE].

[125] R. Brown and N.D. Gilbert, Algebraic models of 3-types and automorphism structures for crossed modules, Proc. London Math. Soc. 59 (1989) 51.

[126] Z. Arvasi and E. Ulualan, 3-types of simplicial groups and braided regular crossed modules, Homol. Homotopy Appl. 9 (2007) 139. 